\title{
BepiColombo Science Investigations During Cruise and Flybys at the Earth, Venus and Mercury
}

\author{
Valeria Mangano ${ }^{1}$ - Melinda Dósa ${ }^{2} \cdot$ Markus Fränz $^{3} \cdot$ Anna Milillo $^{1}$.
}

Joana S. Oliveira ${ }^{4,5}$ - Yeon Joo Lee ${ }^{6}$ - Susan McKenna-Lawlor ${ }^{7}$ Davide Grassi $^{1}$. Daniel Heyner $^{8}$ - Alexander S. Kozyrev ${ }^{9}$. Roberto Peron ${ }^{1} \cdot$ Jörn Helbert $^{10}$. Sebastien Besse ${ }^{11}$ - Sara de la Fuente ${ }^{12}$. Elsa Montagnon ${ }^{13}$. Joe Zender ${ }^{4}$. Martin Volwerk $^{14}$ - Jean-Yves Chaufray ${ }^{15}$ - James A. Slavin ${ }^{16}$ - Harald Krüger ${ }^{3}$. Alessandro Maturilli ${ }^{10}$ - Thomas Cornet ${ }^{11}$ - Kazumasa Iwai ${ }^{17}$ - Yoshizumi Miyoshi ${ }^{17}$. Marco Lucente $^{1}$ - Stefano Massetti ${ }^{1}$. Carl A. Schmidt ${ }^{18}$ • Chuanfei Dong ${ }^{19}$. Francesco Quarati $^{20,21}$ - Takayuki Hirai ${ }^{22}$ - Ali Varsani ${ }^{14}$. Denis Belyaev ${ }^{23}$. Jun Zhong ${ }^{24}$ • Emilia K.J. Kilpua ${ }^{25}$ • Bernard V. Jackson ${ }^{26}$ • Dusan Odstrcil ${ }^{27,28}$. Ferdinand Plaschke $^{14}$ - Rami Vainio ${ }^{29}$. Riku Jarvinen ${ }^{30,31}$. Stavro Lambrov Ivanovski ${ }^{32}$ - Ákos Madár ${ }^{2}$. Géza Erdôs ${ }^{2}$ - Christina Plainaki ${ }^{33}$. Tommaso Alberti ${ }^{1}$ - Sae Aizawa ${ }^{34}$. Johannes Benkhoff ${ }^{4}$. Go Murakami ${ }^{35}$.

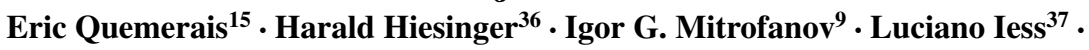
Francesco Santoli ${ }^{1}$ - Stefano Orsini ${ }^{1}$ - Herbert Lichtenegger ${ }^{14}$ - Gunther Laky $^{14}$. Stas Barabash $^{38}$ • Richard Moissl ${ }^{39}$ • Juhani Huovelin ${ }^{25}$. Yasumasa Kasaba ${ }^{40}$. Yoshifumi Saito $^{35}$ - Masanori Kobayashi ${ }^{41}$ - Wolfgang Baumjohann ${ }^{14}$

Received: 13 June 2020 / Accepted: 13 January 2021 / Published online: 11 February 2021

(C) The Author(s) 2021

Abstract The dual spacecraft mission BepiColombo is the first joint mission between the European Space Agency (ESA) and the Japanese Aerospace Exploration Agency (JAXA) to explore the planet Mercury. BepiColombo was launched from Kourou (French Guiana) on

The BepiColombo mission to Mercury

Edited by Johannes Benkhoff, Go Murakami and Ayako Matsuoka

V. Mangano

INAF-IAPS (Institute for Astrophysics and Planetology from Space), via del Fosso del Cavaliere 100, 00133, Rome, Italy

2 Department of Space Research and Space Technology, Wigner Research Centre for Physics, Budapest, Hungary

3 Max Planck Institute for Solar System Research, Goettingen, Germany

4 Science Division, European Space Agency (ESA), European Space Research and Technology Centre (ESTEC), Noordwijk, The Netherlands

5 Space Magnetism Area, Payloads and Space Sciences Department, INTA, Torrejón de Ardoz, Spain

6 Zentrum für Astronomie und Astrophysik, Technische Universität, Berlin, Germany

7 Space Technology Ireland, Ltd., Maynooth, Co. Kildare, Ireland

8 Institut für Geophysik und extraterrestrische Physik, Technische Universität, Braunschweig, Germany 
October 20th, 2018, in its packed configuration including two spacecraft, a transfer module, and a sunshield. BepiColombo cruise trajectory is a long journey into the inner heliosphere, and it includes one flyby of the Earth (in April 2020), two of Venus (in October 2020 and August 2021), and six of Mercury (starting from 2021), before orbit insertion in December 2025. A big part of the mission instruments will be fully operational during the mission cruise phase, allowing unprecedented investigation of the different environments that will encounter during the 7-years long cruise. The present paper reviews all the planetary flybys and some interesting cruise configurations. Additional scientific research that will emerge in the coming years is also discussed, including the instruments that can contribute.

Keywords BepiColombo $\cdot$ Mercury $\cdot$ Venus $\cdot$ Earth $\cdot$ Cruise $\cdot$ Flyby

\section{Introduction}

The dual spacecraft mission BepiColombo is the first joint mission between the European Space Agency (ESA) and the Japanese Aerospace Exploration Agency (JAXA) to explore the planet Mercury. BepiColombo was launched from Kourou (French Guiana) on October 20th, 2018 in its packed configuration named Mercury Composite Spacecraft, MCS) including the two spacecraft: the ESA Mercury Planetary Orbiter (MPO) and the JAXA Mio (formerly named Mercury Magnetospheric Orbiter) (see Benkhoff et al., this journal). Hence, BepiColombo packed configuration (usually named Mercury Composite Spacecraft,

9 Institute for Space Research, Moscow, Russian Federation

10 Deutsches Zentrum für Luft- und Raumfahrt (DLR), German Aerospace Center Institute for Planetary Research, Berlin, Germany

11 Aurora Technology B.V. for ESA, European Space Agency, Madrid, Spain

12 ESA/ESAC, Villanueva de la Canada, Spain

13 ESA/ESOC, Darmstadt, Germany

14 Space Research Institute, Austrian Academy of Sciences, Graz, Austria

15 LATMOS, Université Versailles Saint-Quentin, Guyancourt, France

16 Climate and Space Sciences and Engineering, University of Michigan, Ann Arbor, MI, USA

17 Institute for Space-Earth Environmental Research, Nagoya University, Furo-cho, Chikusa-ku, Nagoya, 464-8601, Japan

18 Boston University, Boston, USA

19 Department of Astrophysical Sciences and Princeton Plasma Physics Laboratory, Princeton University, Princeton, NJ, USA

20 LumMat/RST/AP, Delft University of Technology, Delft, The Netherlands

21 Gonitec BV, Den Haag, The Netherlands

22 Planetary Exploration Research Center (PERC), Chiba Institute of Technology, Chiba, Japan

23 Space Research Institute (IKI), Moscow, Russia

24 Institute of Geology and Geophysics, Chinese Academy of Sciences, Beijing, China

25 Department of Physics, University of Helsinki, Helsinki, Finland 
MCS) is composed by ESA spacecraft MPO, the JAXA spacecraft Mio, the Mercury Transfer Module MTM, and the sunshade cone MOSIF (acronym for Magnetospheric Orbiter Sunshield and Interface Structure) designed to protect Mio during the cruise phase.

BepiColombo cruise trajectory is a long journey into the inner heliosphere, and it includes one flyby of the Earth (in April 2020), two of Venus (in October 2020 and August 2021), and six of Mercury itself (starting from 2021), before the orbit insertion in December 2025.

Figure 1 shows a schematic of the complete cruise trajectory (from launch to orbit insertion), and Fig. 2 shows the composite spacecraft MCS with its reference axes: $+Y$ is directed into the radiator panel of MPO, $+Z$ is along the main axis of MCS, from Mio towards the $\mathrm{MTM} ;+X$ completes the Cartesian 3-axis system.

The MPO payload comprises 11 experiments and instrument suites, and Mio carries 5 experiments and instrument suites. The former will focus on the planet itself and close interactions with its surface, while the latter will study the environment around the planet including the planet's exosphere and magnetosphere, and their interaction processes with the solar wind. However, the MCS configuration will not allow full operability of all instruments onboard. In fact, Mio will be partly shielded by MOSIF, thus allowing instruments to detect signals only within a conical field-of-view around the MCS's $-Z$ axis. On-board the MPO, the instruments with apertures in the $+Z$ axis are obstructed by the MTM and, hence, are not able to acquire scientific measurements. Nevertheless, all the instruments not requiring pointing or with apertures in the other directions will be, in principle, able to operate.

Hence, BepiColombo cruise phase has become an important opportunity to investigate different environments of the inner Solar System, even if it was not originally designed for that purpose.

26 Center for Astrophysics and Space Sciences, University of California at San Diego, La Jolla, CA, USA

27 Department of Physics and Astronomy, George Mason University, Fairfax, VA, USA

28 NASA/GSFC Heliophysics Science Division, Space Weather Laboratory, Greenbelt, MD, USA

29 Department of Physics and Astronomy, University of Turku, Turku, Finland

30 Finnish Meteorological Institute, Helsinki, Finland

31 Department of Electronics and Nanoengineering, School of Electrical Engineering, Aalto University, Espoo, Finland

INAF-OATs (Osservatorio Astronomico di Trieste), Trieste, Italy

Italian Space Agency, Rome, Italy

34 Institut de Recherche en Astrophysique et Planétologie, CNRS, CNES, Université de Toulouse, Toulouse, France

35 Japan Aerospace Exploration Agency, Institute of Space and Astronautical Science, Sagamihara, Kanagawa 252-5210, Japan

Institut für Planetologie, Westfälische Wilhelms-Universität Münster Münster, Germany

Dipartimento di ingegneria meccanica e aerospaziale, Università La Sapienza, Roma, Italy

38 Swedish Institute of Space Physics, Kiruna, Sweden

39 Research and Scientific Support Department, ESTEC, Noordwijk, The Netherlands

40 Planetary Plasma and Atmospheric Research Center, Graduate School of Science, Tohoku University, Sendai, Miyagi 980-8578, Japan 
Fig. 1 BepiColombo spacecraft sketch of different cruise phases: launch in 2018, MCS orbit around the Earth, Earth's flyby, cruise, Venus flybys, Mercury's flybys, separation, and orbit insertion of the MPO and Mio spacecraft in 2026 after separation of the 4 components of MCS (courtesy of Airbus Defense and Space)

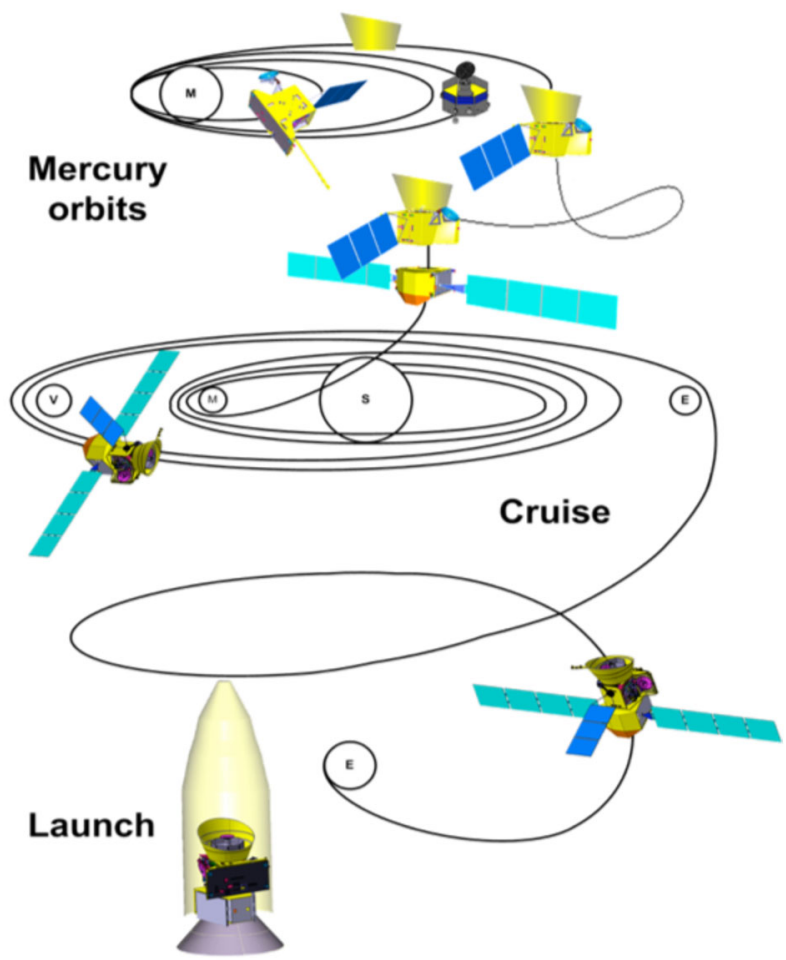

The present paper aims to review all the additional science investigations that may take place during the planetary flybys and during the cruise in specific configurations with other spacecraft.

Section 2 will discuss the cruise phase, including attitude details and limitations of the MCS configuration, and focus on the possible scientific objectives to be addressed during the cruise phase in general. It will include discussions on multi-spacecraft coordinated observations, and a brief description of how the present space weather modeling tools may help in predicting and interpreting the data.

Section 3 will focus on the radio science experiments which are the only ones originally planned during the cruise of BepiColombo. Section 4 will report on the Earth flyby investigations that occurred on April 10th 2020. Section 5 will give an overview of the planet Venus characteristics in terms of atmosphere and induced magnetosphere, and will describe in detail the possible Venus investigations during the two flybys with the instrumentation onboard MCS in operation. Finally, Sect. 6 will focus on the Mercury flybys, from October 2021 to January 2025 , being with the first approach to the planet. The primary goal of the BepiColombo mission starting from 2026 is to study Mercury and its environment, when the two spacecraft will be in their own orbits, with the instrumentation onboard fully operative. Nevertheless, the peculiar geometry of the flybys will allow first investigations of Mercury exosphere, intrinsic magnetic field and magnetosphere, and also surface and interior starting from 2021 , i.e. 5 years in advance with respect to the nominal mission.

41 Planetary Exploration Research Center, Chiba Institute of Technology, 2-17-1, Tsudanuma, Narashino, Chiba 275-0016, Japan 


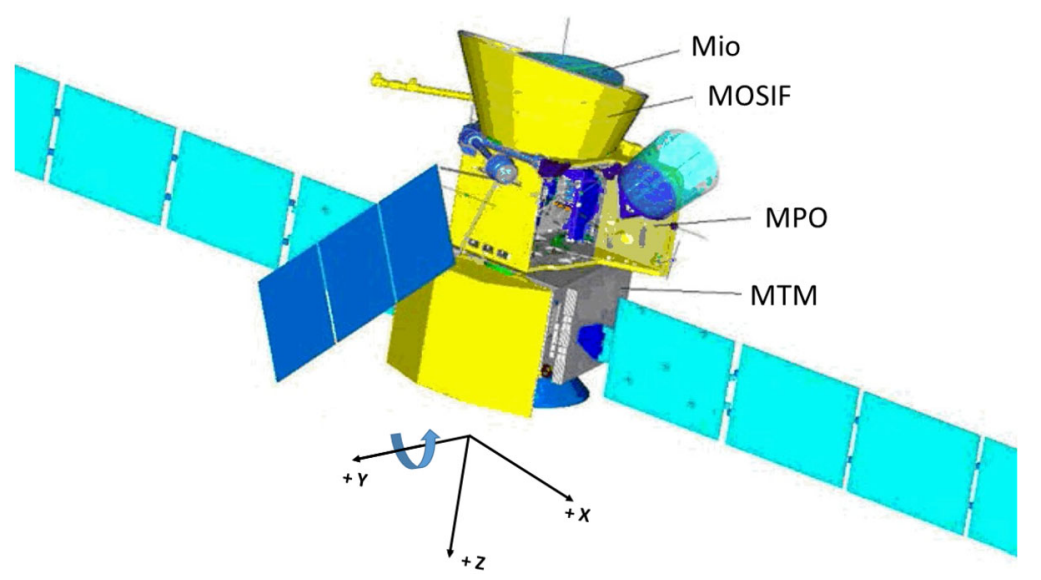

Fig. 2 The composite spacecraft MCS during cruise and its reference frame $X Y Z$ : $Y$-axis points towards the Sun (and MPO radiator - not visible in this image - points towards $-Y$ axis); $+Z$-axis is along the main axis of the spacecraft pointing at the anti-MTM direction (i.e., Mio - or MMO - axis is pointing towards $-Z$ ), and $+X$ axis completes the right hand coordinate system. Note that the magnetometer boom of MAG and the antenna used by MORE are both fully deployed during cruise (courtesy of Airbus Defense and Space)

Section 7 will summarize the different scientific tasks that BepiColombo may contribute to investigate during its cruise to Mercury, together with our conclusions. An Appendix will complement the present paper, to provide the reader with other useful information related to the wide field of investigations discussed.

\section{Cruise}

During the interplanetary cruise phase, the radiator panel of MPO $(-Y$ axis $)$ is pointing anti-sunwards (see Fig. 2), thus $+Y$ axis is directed towards the Sun. Only small deviations from this pointing described by solar aspect angle (SAA), that is the angle between $+Y$ axis and the Sun direction projected onto the $X Z$ plane are allowed during the cruise phase. The allowed deviations are between $+47^{\circ}$ and $+17^{\circ}$ depending on the spacecraft distance to the Sun (see Table 9 in the Appendix).

Orbit changes are achieved by the planetary flyby maneuvers and by firing of the Solar Electric Propulsion System into specific periods (see Table 10 in the Appendix). The spacecraft attitude is controlled around the Sun line such that the angular momentum loading is minimized while ground contact is maximized during the ground station passes. This results in tight attitude constraints, as well as limited communication windows with the High Gain Antenna, typically only up to a maximum of 10 hours per day.

Hence, during the cruise phase, unobstructed instrument operations are mainly limited by the following constraints:

1) as a baseline, no instrument operations are planned during solar electric propulsion, both for MPO and Mio;

2) as BepiColombo moves away from the Earth, flexibility in attitude and the downlink capability will further limit possible instrument operations.

Since the launch, and outside the solar electric propulsion periods, onboard the MPO, the magnetometer MAG (Glassmeier et al. 2010), the Italian Spring Accelerometer ISA (Santoli 
et al. 2020), the radio science experiment MORE (Iess et al. 2021, this journal), the Mercurian Gamma-ray and Neutron Spectrometer MGNS (Mitrofanov et al. 2010; Mitrofanov et al. 2021, this journal), and the environmental radiation sensor BERM (Moissl et al., this journal) are fully operative.

The X-ray and particle spectrometers of SIXS (Huovelin et al. 2020, this journal) and the UV-spectrometer PHEBUS (Quémerais et al. 2020, this journal) will be operated throughout the cruise phase depending on dedicated feasibility analysis.

The magnetometer MAG onboard MPO is mounted on the fully deployed $2.8 \mathrm{~m}$ boom at the edge of the radiator ( $-Y$ axis) and the $X$ axis side, and the antenna used by MORE is fully deployed too (see Fig. 2). The MGNS spectrometers are located inside the MPO spacecraft structure and measures gamma-rays and neutrons, however during cruise these measurements are impacted by the surrounding material of the composite stack. The ISA accelerometer is also inside the spacecraft structure but does not need pointing and is fully operative.

The BERM sensor is mounted behind the radiator panel and looks to the $-Y$ direction, thus always anti-sunward. The three SIXS-X X-ray detectors are oriented towards the $+Y$ direction (unfortunately with an offset such that none of the detectors has the Sun in its fieldof-view during the cruise phase). The SIXS-P particle detector is operational and views in five orthogonal directions (three of them not obstructed during cruise). The PHEBUS baffle is located at the radiator panel, and can rotate $360^{\circ}$ around the $-Y$-axis. The infrared spectrometer MERTIS (Hiesinger et al. 2020, this journal) can be operated by its calibration telescope also pointing in $-Y$-direction. Finally, the two ion sensors of SERENA are on the edge of the $-X$ axis, and can be operated too (the other two neutral sensors in the $+Z$ face being blocked by the MTM). Mio instrumentation is only partially obstructed by the sunshield, and have a free field of view of about 30 degrees in the $-Z$ direction (Murakami et al. 2020). MPPE sensors (HEP-e, ENA, MSA, MIA, and MEA) (Saito et al. 2021, this journal) and MDM (Kobayashi et al. 2020) have some open field of views around $-Z$ axis above MOSIF. The MGF (Baumjohann et al. 2020) and PWI (Kasaba et al. 2020; Yagitani et al. 2020; Karlsson et al. 2020) instruments are not deployed yet; hence, noise levels are higher during the cruise phase than during the Mercury orbit phase. Table 1 summarizes the instruments viewing directions, field of views and constraints valid during the cruise phase.

\subsection{Scientific Objectives}

During the long cruise of BepiColombo in the inner Solar System, the only official science goal originally planned was the superior solar conjunction experiment to be performed by MORE (see Sect. 3 and Iess et al. 2021, this journal). Nevertheless, in recent years it was clear that the opportunity to operate the other available instruments should be exploited.

During the BepiColombo cruise phase (2018-2025), the solar cycle will be in its ascending phase with predicted solar maximum predicted in July 2025. This fact will provide the opportunity to study interplanetary physics under different conditions of solar activity (Fig. 3).

Even limited instrument operations of BepiColombo can contribute to a wide range of scientific investigations, as for example:

- In-situ solar wind observations on small scales and large scales, with special regard to the study of transient events such as Coronal Mass Ejections (CMEs), and Solar Energetic Particles (SEPs), but also background solar wind and scale-invariant properties (turbulence, intermittency, MHD instabilities), high speed streams, Co-rotating Interaction Regions (CIRs), and Heliospheric Current Sheet (HSC). 


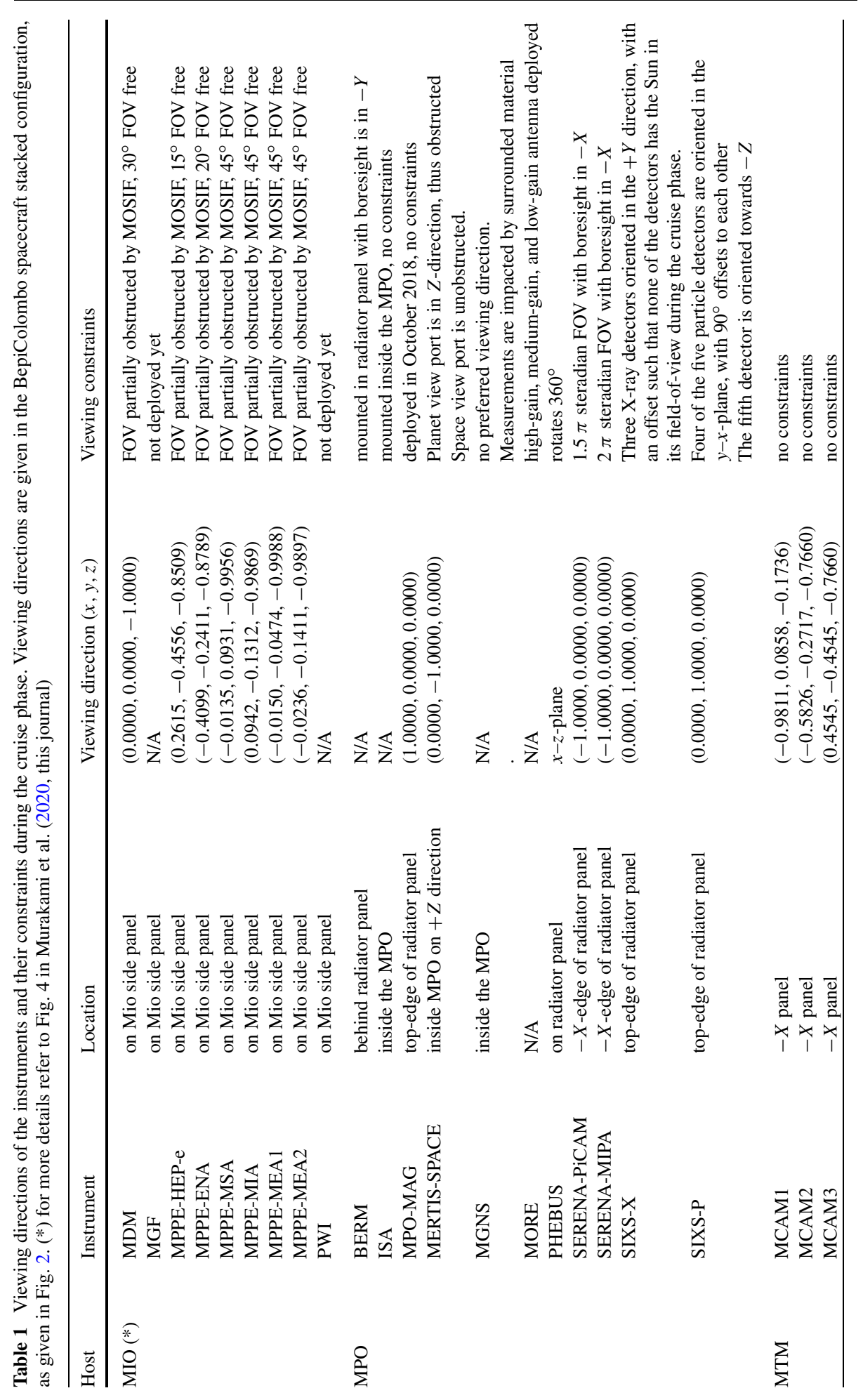


Fig. 3 Solar cycle 25 forecast update compared to previous ones as released on December 2019 by NOAA. The rising phase of cycle 25 is expected to peak in July 2025 (courtesy of NOAA)

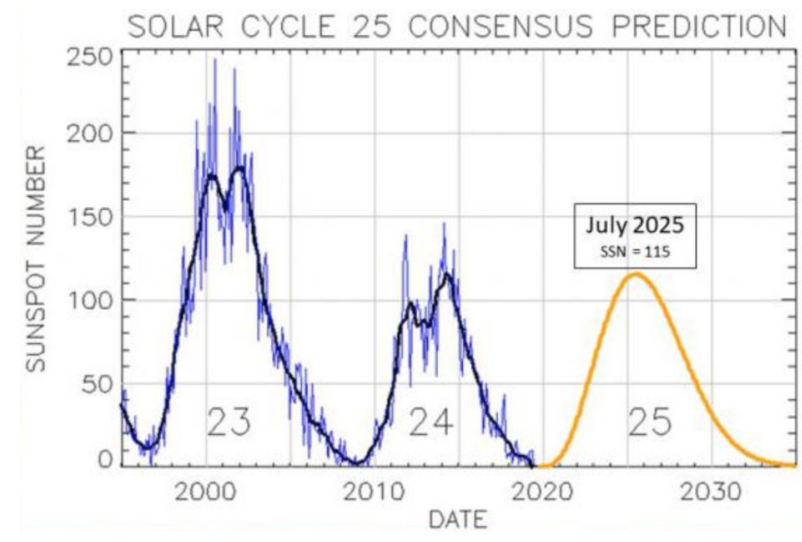

- Measurements of the plasma composition, ion flux and density, and magnetic fields in the vicinity of Venus and Mercury during flybys (see devoted Sects. 5 and 6).

It has to be emphasized, that while MPO and Mio will have polar orbits around Mercury, the flybys will occur at low-latitudes. These are the best opportunities to study low-latitude solar wind interaction with the magnetosphere, Kelvin-Helmholtz boundary waves and important internal dynamics like plasma sheet convection and reconnection.

- coordinated science observations together with other spacecraft in Earth orbit and in the inner Solar System, e.g., Akatsuki, Parker Solar Probe, SDO, Proba-2, Hisaki, SOHO, Solar Orbiter and JUICE, to provide measurements from additional vantage points;

- observations of InterPlanetary Scintillations (IPS) coordinated with Earth-based measurements to provide information on solar wind density;

- analysis of cometary composition, and detection of dust particles of different origins;

- monitoring the local radiation background due to bombardment by energetic particles of Galactic Cosmic Rays;

- Gamma Ray Burst (GRB) detection and localization;

- and, of course, the above mentioned Superior Solar Conjunction measurements (SSC) to test general relativity.

\subsubsection{Solar Wind and Interplanetary Magnetic Field}

The solar wind is a continuous flow of charged particles that originates from the Sun's corona. The magnetic energy contained at lower regions rises to the higher corona and dissipates via processes not yet understood in detail, causing coronal heating and solar wind acceleration (e.g. Shoda et al. 2019). As solar wind propagates radially outward, it fills up the heliosphere and interacts with various obstacles (e.g. planetary bodies). The parameters of solar wind plasma and its dynamic changes determine all plasma processes in space. Also the Interplanetary Magnetic Field (IMF) carried by the solar wind is prone to various kinds of structures and discontinuities on a broad range of scales (see e.g. Burlaga et al. 1969) from the large scale ones, like CMEs and CIRs, to small scale structures like Magnetic Holes (MHs) and Mirror Mode (MM) waves, via middle scale reconnection structures and flux tubes.

In particular, BepiColombo can provide a huge dataset of the interplanetary magnetic field through the almost continuous measurements of MPO-MAG, plus localized (in time and space) measurements of the solar wind parameters like density and velocity by other ion sensors, and radiation at other wavelengths through the $\mathrm{X}$ - and gamma-sensors onboard. 
Small Scale Heliospheric Structures Magnetic holes (MHs) were first detected in the Explorer 34 (IMP I) data by Turner et al. (1977), and identified as depressions in the solar wind magnetic field strength with $|B|<1 \mathrm{nT}$ in a background field around $6 \mathrm{nT}$ and a very small rotation of the magnetic field over the structure $\left(\theta<10^{\circ}\right)$. MHs were assumed to be diamagnetic, created by inhomogeneities in the solar wind, but the plasma data for these structures were insufficient to actually prove this assumption. Later studies (e.g. Stevens and Kasper 2007) have shown that MHs mainly occur in high solar wind plasma- $\beta$ regions, which makes them similar to Mirror Mode (MM) waves (Hasegawa 1969). MMs occur in high- $\beta$ plasmas when there is a temperature asymmetry. This MM instability usually leads to non-propagating wave trains of structures where the magnetic field and plasma density oscillate in anti-phase, which are transported by the plasma flow. The MHs seemed to occur mainly in MM stable regions of the solar wind (Stevens and Kasper 2007) and wave trains mainly appear in MM unstable plasma regions (Winterhalter et al. 1995). This may lead to the hypothesis that MHs are the final stages of MMs. In addition, Hasegawa and Tsurutani (2011) proposed a Bohm-like diffusion mechanism (Bohm and Gross 1949), in which the higher frequencies of the structure diffuse faster than the lower ones, which results in larger magnetic structures. This mechanism was shown to happen also in Venus's magnetosheath and in comet 1P/Halley's coma (Schmid et al. 2014).

Recent investigations of linear MH occurrence rates using MESSENGER magnetometer data (Anderson et al. 2007) during the cruise from Venus to Mercury in 2007-2011 were performed (Volwerk et al. 2020). The results show a gradual decrease in the occurrence rate of linear MHs from Mercury $(\sim 14 \%)$ to Venus $(\sim 10 \%)$. This agrees with the observation that MHs in the solar wind occur between $\sim 0.1$ to $\sim 7.5$ per day at different locations in the solar system. Hence, an analysis throughout the cruise, in particular before and after Venus flybys, would be of particular interest to better study the magnetic holes characteristics. With the MPO-MAG boom deployed and the instrument operational, the detection and measurements of MHs can be repeated through BepiColombo's cruise phase, as done during the MESSENGER cruise phase (years 2004-2011).

In particular, this type of measurements by BepiColombo may fill the gap of magnetic data at heliospheric distances between Earth and Venus that MESSENGER did not acquire. In addition, magnetic field measurements will enable the study of, e.g., the solar wind turbulence (such as its evolution with the heliocentric distance), the energy transfer from large to small scales (by looking at the behavior of magnetic field spectrum, e.g. Korth et al. 2011), the role of stochastic fluctuations, magnetic reconnection, intermittency, selforganized structures and waves on spectral and scaling properties (e.g. Dong et al. 2018b). Concerning the radial evolution of solar wind turbulence we will understand more deeply the role of non-linearities during the expansion phase of the solar wind (Tu and Marsch 1995), i.e., a key feature for properly characterizing energy transfer across different scales and at different heliocentric distances (Alberti et al. 2020; Chen et al. 2020). Moreover, we will also have the opportunity to investigate small-scale processes (e.g., sub-ion and ion-kinetic effects) as well as some characteristic phenomena of turbulent flows as coherent structures, i.e., plasma regions of concentrated vorticity, intermittency, i.e., the manifestation of sudden field changes manifesting in field gradients and anomalous scalings of the field increments, and the validity of an exact relation for the scaling of the third-order moment of fluctuations, e.g., the Yaglom law, at various heliocentric distances (e.g., Bruno and Carbone 2016).

Large Scale Heliospheric Structures: Transients The understanding of the properties and evolution of large scale interplanetary structures is one of the major challenges in current heliospheric research. This is largely due to the fact that only limited observations have been 
available with varying heliospheric distances as most measurements come at or near the Earth orbit, plus the Helios missions in the 1970 and occasional measurements by planetary missions, such as MESSENGER and Venus Express (Schwenn 1990; Bothmer and Schwenn 1998; Winslow et al. 2015; Good and Forsyth 2016).

Particular attention should be put on:

- Interplanetary Coronal Mass Ejections (ICMEs). ICMEs are the interplanetary counterparts of coronal mass ejections (CMEs) and they are strongly distorted by their interaction with the background solar wind which determines significantly their evolution. The overall structure of the resulting interplanetary disturbance is very complex. ICMEs are important drivers of space weather events not only in the circumterrestrial space but also at planetary environments (see discussions in Lilensten et al. 2014; Plainaki et al. 2016; Kilpua et al. 2017, and references therein).

The integral part of the ICME is a magnetic flux rope that consists of bundles of helical magnetic field lines that wind about the common axis (e.g., Klein and Burlaga 1982; Burlaga 1988; Exner et al. 2018). When sufficiently faster than the ambient solar wind, ICMEs drive fast forward leading shocks through the solar wind and a turbulent sheath forms between the shock and the ICME leading edge. ICME-driven sheaths are compressive structures, characterized by large amplitude magnetic field variations and large dynamic pressure. ICME flux ropes are associated with smoothly changing magnetic field direction and lower dynamic pressure. The global morphology of ICMEs and the nature of interactions between multiple ICMEs and between ICMEs and SIRs (Stream Interaction Regions) is still unresolved (e.g., whether they are generally inelastic, elastic or super-elastic and how the structure and kinematics of the ICMEs involved are affected, see Lugaz et al. 2017). Such issues could benefit from multi-point observations (see Sect. 2.2.1). Sometimes interactions can occur relatively quickly e.g., as featured by Kilpua et al. (2019) who investigated a case where two ICMEs were just about to start interacting at Venus, but by reaching the Earth had coalesced together, resembling a single coherent ICME flux rope, and the shock of the second ICME had also propagated past the preceding ICME.

Another open question is how CMEs that start as more or less coherent flux ropes at the Sun, often transform into quite complex ICMEs in interplanetary space (e.g., Dasso et al. 2007; Kilpua et al. 2013). On average, only about one-third of ICMEs near Earth orbit exhibit clear flux rope signatures (e.g., Cane and Richardson 2003), while the rest have more disorganized field characteristics.

The lack of flux rope signatures can be explained either by interplanetary evolution, e.g. magnetic flux erosion (e.g., Ruffenach et al. 2015), or by the spacecraft's crossing the ICME far from the center. Another possible reason can be that interactions occur with other large scale heliospheric structures (e.g., Manchester et al. 2017; Winslow et al. 2016). This is an additional reason why more measurements are needed.

In fact, observations made at the varying heliospheric distances may help clarify the physical mechanisms of the evolution/interactions of ICMEs and how they progressively change their structure. Since the BepiColombo cruise phase coincides with the ascending and maximum phases of Solar Cycle 25, a high number of ICMEs (though not predictable) are expected to be encountered.

- Solar Energetic Particles (SEPs). SEPs are mainly protons of solar origin and, to a lesser extent, heavier ions; they are accelerated to energies up to hundreds of $\mathrm{MeV}$ (and in some rare cases also GeVs) (Miroshnichenko 2018), either at the flare reconnection sites or at the shock regions driven by CMEs propagating through the solar corona in the interplanetary space (e.g., Desai and Giacalone 2016, and references therein). SEPs have been 
observed at Mercury, Earth, and Mars and are important manifestations of space weather processes.

In the most extreme cases, they can fill the whole inner heliosphere with greatly enhanced fluxes of energetic electrons, protons and heavy ions (e.g., Dresing et al. 2014), that can last from days up to weeks. There are still several key open questions on their acceleration and transport that the inner heliospheric probes Solar Orbiter (ESA) and Solar Parker Probe (NASA) can help to answer, as it was done recently, when the combination of ACE, MESSENGER, STEREO-A, B, MAVEN, and Juno data at widely separated heliospheric longitudes was used to study:

(a) the cross-field diffusion processes in both the solar corona and interplanetary space,

(b) broad particle sources associated with coronal and interplanetary shocks initially driven by CMEs, and

(c) complex magnetic field configurations in the corona or interplanetary space that allow SEPs injected from a narrow source to reach distant locations (Lario et al. 2016).

In particular, comparison of SEP flux time-profiles and variations of the energy spectrum at different locations can provide crucial knowledge on whether the shocks are able to accelerate and inject SEPs into different longitudes, and whether cross-field diffusion causes wide spreading SEPs at different heliospheric longitudes. In addition, the combined studies of in-situ data, type-II radio burst data (e.g., Iwai et al. 2020), and global MHD simulations may provide clues on the structures of the background interplanetary magnetic fields and propagation characteristics of CMEs. Such clues are important to specify the acceleration regions of SEPs, and the 3D field line connection close to the solar surface from any specified in-situ locations including planets or a spacecraft.

Large Scale Heliospheric Structures: Background Solar Wind Solar wind outflow is continuous, and plasma velocity is usually defined either as "slow" $(\sim 400 \mathrm{~km} / \mathrm{sec})$ or "fast" $(\sim 700 \mathrm{~km} / \mathrm{s})$, even though showing wide range of values. In regions where slow and fast solar wind meet, solar wind SIRs (Richardson 2018) are formed. SIRs develop with radial distance, either the fast wind overtaking slow wind (compression region), or slow wind lagging behind fast wind (rarefaction region).

SIRs are also often named Co-rotating Interaction Regions (CIRs) to reflect their repeating nature with solar rotation on 27 days as well as on short-term harmonics ( 7 days, 13.5 days), especially during solar minimum conditions.

A typical SIR fingerprint shows a sudden and well-marked increase in density, temperature, and magnetic field strength and a constant change of velocity over the interface. The azimuthal component of the magnetic field has an opposite sign on each side of the interface, such as the non-radial components of the velocity vector.

Long-term observations of SIRs as they co-rotate with the Sun give us insight into coronal properties as well. The source of CIRs lies in the corona on the border of slow and fast solar wind sources. The study of typical CIR features by Dósa and Erdős (2017), e.g., alternating deviation of the velocity vector, magnetic flux density enhancements, etc., during the 23rd solar cycle, evidenced that during the calmer decreasing phase the source of the emerging CIRs were located on constant heliographic longitudes. Two exceptionally strong features during 2007-2008 are visible in Fig. 4. They appear in the magnetic field dataset as inclined red stripes around Carrington longitudes $-180^{\circ}$ and $-60^{\circ}$, and also in the velocity deviation dataset around $180^{\circ}$ and $300^{\circ}$. The coronal source longitude on the other hand rotated with a different rotation rate than the Carrington rotation (faster during the declining phase), but showed a constant trend, giving us some hints of coronal mechanisms. This 
Fig. 4 Source longitude of CIRs over the long term: unsigned magnetic flux density and solar wind azimuth angle (the angle between the velocity vector and the radial direction) measured by ACE during cycle 23 mapped back to the longitude of origin. Each horizontal line corresponds to 25.3802 days of data (time period corresponding to the Carrington rotation). Year to the right, Carrington rotation number to the left ( $y$ axis). Inclined red stripes are visible, implying that magnetic field enhancements (occurring at the same time as the deviation changes from positive to negative) recurred later or earlier than the Carrington rotation rate (from Dósa and Erdős 2017)

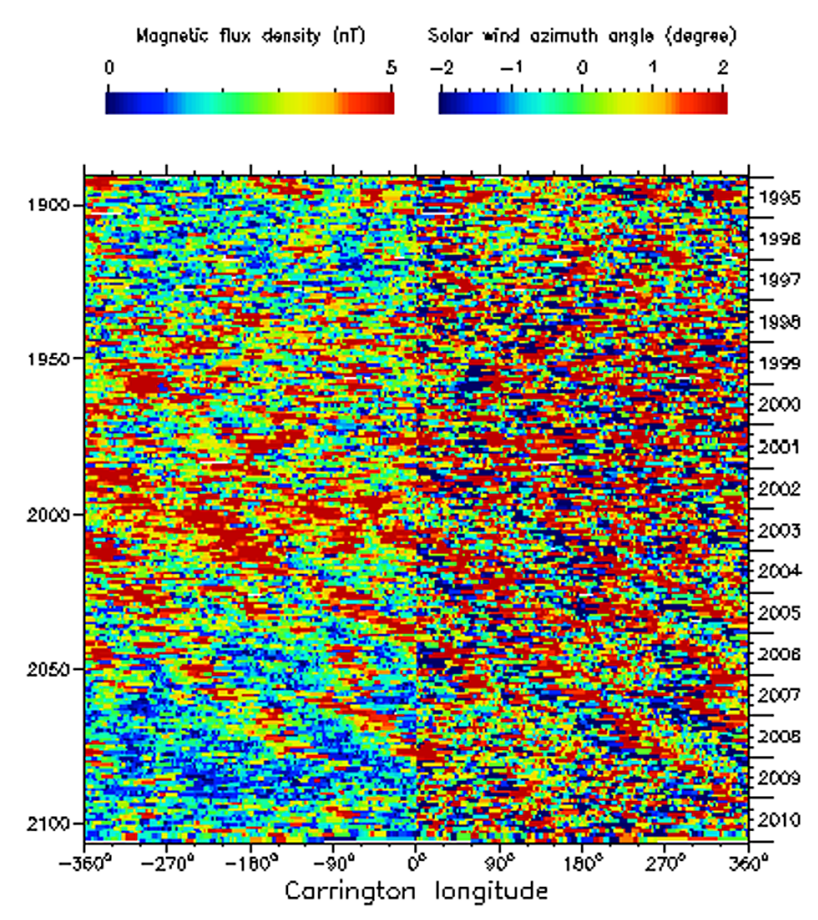

finding should be further investigated in relation to active longitudes identified on the photosphere. Propagation properties of CIRs can be best studied when observed at different radial distances: especially the relationship between the magnetic sector boundaries and SIRs, but also the 3-dimensional evolution of the SIR feature.

Interfaces of slow and fast solar wind streams are often, but not necessarily located at magnetic sector boundaries. Dósa and Erdős (2017) also showed that during the years 20022006 the Heliospheric Current Sheet (identified as magnetic sector boundaries at $1 \mathrm{AU}$ near the Earth) rotated also somewhat faster than the Carrington rate (25.3802 days per sidereal rotation) but with a different rate than the CIRs. Examining the rotation speed of the different features at different radial distances could enhance our understanding of how these features evolve in time and space, and what is their exact relationship with each other.

\subsubsection{Dust and Comets}

The study of dust distribution in the inner Solar System is important to understand the evolution of interplanetary dust travelling towards the Sun due to solar radiation drag (PoyntingRobertson effect), as well as a better constraint on the inner Solar System environment, both chemically and dynamically. Very few observations of dust in the inner Solar System are currently available. In the 1970s the Helios spacecraft observed the interplanetary dust distribution between 0.3 AU and 1.0 AU from the Sun for the first time (Grün et al. 1980). Analysis of these data led to the discovery that an interstellar dust stream originated from outside the Solar System and penetrates into the inner heliospheric region (Altobelli et al. 2006). Only recently, the JAXA's solar power sail demonstrator IKAROS provided new data by its large-area dust impact sensor, ALADDIN (Yano and Hirai 2016), and revealed a $10 \mu \mathrm{m}$-sized interplanetary dust distribution from 1.1 AU to $0.72 \mathrm{AU}$ (at Venus orbit). It is 
Fig. 5 Field of view of MDM during the cruise phase. The unobstructed field of view will be of $30^{\circ}$ at maximum, and it derives from the relative positions of MDM, Mio, and MOSIF

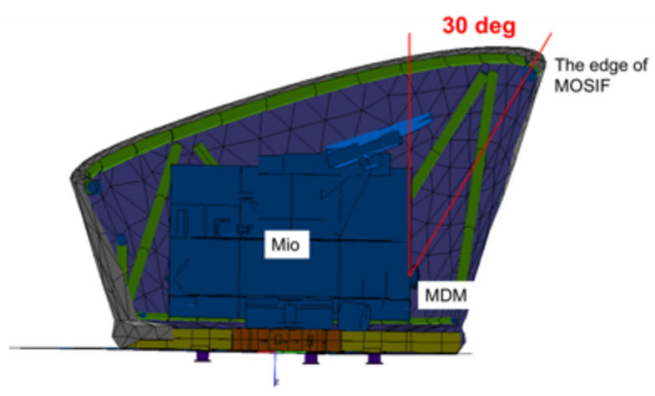

noteworthy that on 2nd September 2019, a comet-like object 322P/SOHO passed its perihelion flying to a heliocentric distance of $0.12 \mathrm{AU}$, and swept by Parker Solar Probe (PSP) at a relative distance as close as $0.025 \mathrm{AU}$, where images photographed by WISPR (Wide-field Imager for Solar Probe Plus) onboard PSP shows messy trails caused by dust bombardment (He et al. 2020). The 7-year cruise phase of BepiColombo, hence, is a precious opportunity for in-situ measurement of cosmic dust including cometary dust trail particles in the inner Solar System. The dust impact sensor MDM on a side panel of the Mio spacecraft will be able to provide measurements of dust particles with diameter of $\sim 1 \mu \mathrm{m}$ (at an impact velocity of $10 \mathrm{~km} / \mathrm{s}$ and dust material density of $2.0 \mathrm{~g} / \mathrm{cm}^{3}$ ) (Kobayashi et al. 2020). In fact, though it is surrounded by the MOSIF (see Figs. 2 and 5), there is a possibility to detect dust impacts through the MOSIF's topside aperture which partially provides the MDM sensor with $\sim 30^{\circ}$ field of view (see Fig. 5).

In addition, the MDM sensor, which is made of piezoelectric ceramics, can probably detect also acoustic vibrations propagating through the MOSIF wall and possibly the whole BepiColombo spacecraft, induced by impacts of relatively large dust particles, e.g. $>100 \mu \mathrm{m}$ in diameter. For instance, the spacecraft passage through a comet dust trail (consisting mainly of large millimeter-sized dust particles emitted from the parent comet), will allow MDM to measure the small vibration of the spacecraft structure generated by the impacts. Capitalising on its high sensitivity, also the ISA accelerometer can likely contribute to this research topic. Although not directly exposed to outer space, ISA working principle would allow detecting eventual structural vibrations induced by particle/dust impacts propagating along the spacecraft. In this perspective, a synergy with MDM instrument can be envisaged to support the identification of eventual impact events (see Sect. 3.2).

Simulations by the new universal model IMEX (Interplanetary Meteoroid Environment for eXploration) of the recently created cometary dust trails of different comets in the inner Solar System (Soja et al. 2015a,b) may help to study cometary dust trails, as measured by insitu detectors and observed in infrared images, and program in advance devoted observations in particular periods of the BepiColombo cruise. Large variations in the predicted dust fluxes from comet to comet have to be expected because the ejection velocity, mass distribution, and dust production rate - all parameters of the IMEX model - likely vary for each comet and are not well constrained for many comets yet. This may be improved in the future for the comets found in the present analysis to yield more reliable flux predictions. Figure 6 shows the expected dust flux simulated with IMEX for cometary trails crossed by BepiColombo during the cruise. By far the largest flux is encountered in the trail of comet $2 \mathrm{P} /$ Encke. Fluxes predicted for the crossings of other cometary trails are at least two orders of magnitude lower. 

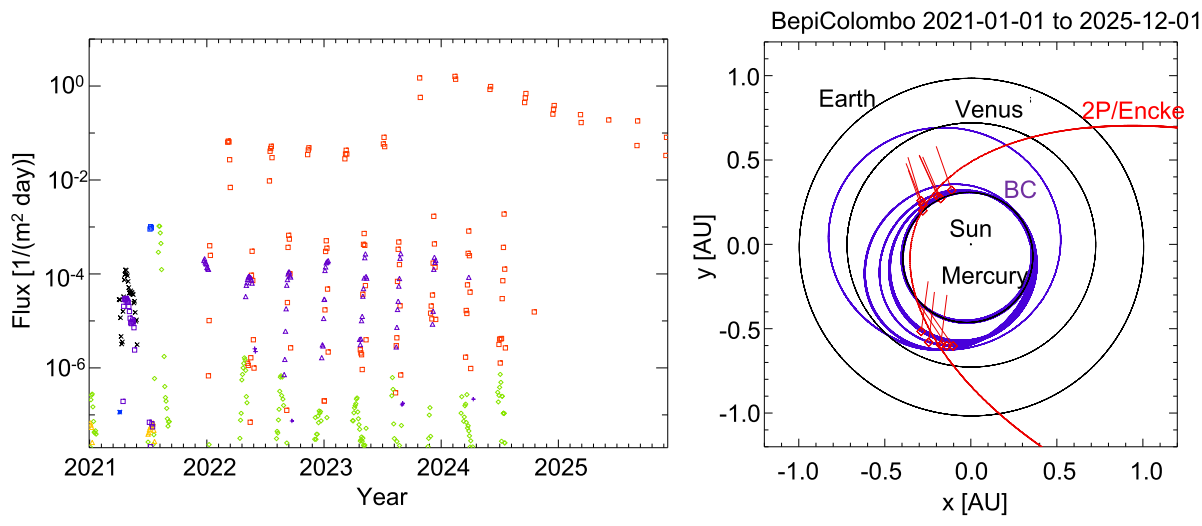

Fig. 6 Left: Simulated fluxes of particles larger than $100 \mu \mathrm{m}$ for cometary trails crossed by the BepiColombo spacecraft during its interplanetary cruise to Mercury. The comet 2P/Encke (red square) shows the largest flux after 2022 when the spacecraft orbits the Sun inside Venus' orbit. The simulations were performed with a step size of two days. Right: BepiColombo interplanetary trajectory from 2021 to 2025 (blue). The orbit of comet $2 \mathrm{P} /$ Encke is shown in red; diamonds indicate BepiColombo's crossings of comet Encke's dust trail as derived from the IMEX model, and red lines attached to the diamonds show the approach direction (speed vector) of trail particles on to the spacecraft. The $X-Y$ plane is the ecliptic plane with vernal equinox oriented towards the $+X$ direction

The flux peaks are rather narrow in space/time with a typical peak width of only several days (simulations in Fig. 6 have a two-day step size). On the right side of Fig. 6, the geometry of BepiColombo's orbit and the orbits of Encke are shown. The impact directions of Encke trail particles on to the BepiColombo spacecraft are indicated for a few crossings of this comet's trail. Maximum fluxes of 1 event $/ \mathrm{m} 2 /$ day are reached for trail crossings in October 2023 and February 2024. These fluxes should be detectable with the MDM dust detector. In addition, during the period of maximum flux contemporary observation for the comet's hydrogen coma can be possibly performed by the PHEBUS instrument (see below and in Fig. 5). If this will be confirmed, it would be the first coordinated observation of in-situ cometary dust trail and coma observation in the inner Solar System.

The study of cometary composition is an important goal that could be addressed by BepiColombo during its cruise phase. One or two comets per year are visible in the inner heliosphere, on average. Hence, a total of about 10 comets could be observable during the 7-year cruise to Mercury. This is an observation goal during the cruise of the UV spectrometer PHEBUS. For each target, it will be possible to compute the time when it intersects the PHEBUS instrument FOV without a change of the spacecraft attitude. Depending on the brightness of the object and its distance to the spacecraft, observations of the hydrogen coma at Lyman- $\alpha$ (EUV) may be done, as done regularly by SWAN/SOHO (Bertaux et al. 1995), to derive the water production rates (e.g., Combi et al. 2018). If possible, OH (FUV line at $308 \mathrm{~nm}$ ) and other weaker emissions could also be observed, as done in the past by SPICAV on Venus Express (Chaufray and Bertaux 2015). Comet 2P/Encke should reach its perihelion on 26 June 2020 and 23 Oct 2023. While the geometry is not favourable for observations near June 2020 by PHEBUS, observations will be possible at the end of November 2023. At the end of November 2023, slightly after the perihelion, the comet-sun distance will be $\sim 1$ AU and the comet-BepiColombo distance equal to $0.6 \mathrm{AU}$ (see Fig. 7). This will allow optimal observation of the comet tail, while the MDM measurements 1 month earlier and 3 months later will be able to detect the dust amount originated from the comet. 
Fig. 7 Geometry of possible PHEBUS observation of comet 2P/Encke in November 2023. Blue cross is for BepiColombo at that date, and black dashed line indicates the direction of PHEBUS observation crossing the trajectory of cope $2 \mathrm{P} /$ Encke (in red)

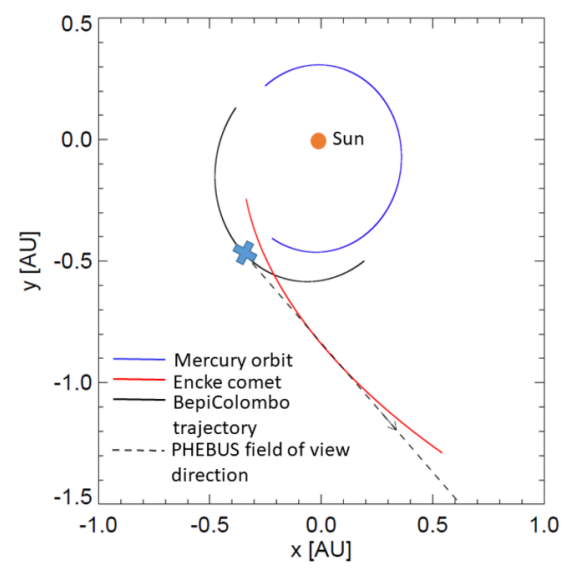

\subsubsection{Other Science Goals: MGNS}

The Mercurian Gamma-ray and Neutron Spectrometer (MGNS) is a scientific instrument developed to study the elementary composition of Mercury's sub-surface by measurements of neutron and gamma-ray emission of the planet. MGNS measures neutron fluxes in a wide energy range from thermal energy up to $10 \mathrm{MeV}$ and gamma-rays in the energy range of $300 \mathrm{keV}$ up to $10 \mathrm{MeV}$ with energy resolution of $5 \%$ at $662 \mathrm{keV}$ and of $2 \%$ at $8 \mathrm{Mev}$ (Mitrofanov et al. 2021, this journal) thanks to the innovative crystal of $\mathrm{CeBr}$ (Kozyrev et al. 2016). During the cruise to Mercury, it is planned that the MGNS instrument will operate continuously (excluding solar electric propulsions periods) to perform measurements of neutrons and gamma-ray fluxes for achieving two main goals of investigations:

1. monitoring of the radiation background of the prompt spacecraft emission due to bombardment by energetic particles of Galactic Cosmic Rays. This data will be taken into account at the mapping phase of the mission on the orbit around Mercury. Detailed knowledge of the spacecraft background radiation during the cruise will help to derive the data for neutron and gamma-ray emission of the planet during the nominal phase at Mercury starting from 2026. In fact, many elements like Si, O, Al, C, Mg, and others the abundance of which at the uppermost layer of the planet is studied - are also present in the material of the spacecraft (Evans et al. 2017). Figure 8 shows the spectrum of the gamma-ray background of the spacecraft measured during the initial part of the cruise. Indeed, the nuclear lines of $\mathrm{Al}, \mathrm{Mg}$, and $\mathrm{O}$ are well-pronounced in the spectrum, which are also expected to be detectable in the gamma-ray spectrum of the Mercury emission.

2. participation in the InterPlanetary Network (IPN) program for the localization of sources of Gamma-Ray Bursts in the sky (Hurley et al. 2013). The localization accuracy by the interplanetary triangulation technique is inversely proportional to the distance between the spacecraft that jointly detected a GRB. Before the launch of BepiColombo, the IPN network included a group of spacecraft in the near-to-Earth orbit (e.g., Konus-Wind, Fermi-GBM, INTEGRAL, Insight-HXMT) and the Mars Odyssey spacecraft on orbit around Mars. Now, MGNS provides an additional interplanetary location, potentially increasing the accuracy of GRBs localization. During the first 13 months of continuous operation, MGNS detected 24 GRBs. Since November 2019 the pre-set time resolution of 20 seconds for GRB profiles measurements was increased into 1 second, and downlink resources allocated. Since then, the corresponding GRB detection rate is increased to 
Fig. 8 An example of MGNS gamma-ray spectrum measured during cruise (accumulation time of 8750 minutes)
Fig. 9 Time profile of gamma-ray burst GRB191125A detected by MGNS gamma-ray spectrometer (1 s acquisition integrated counts)
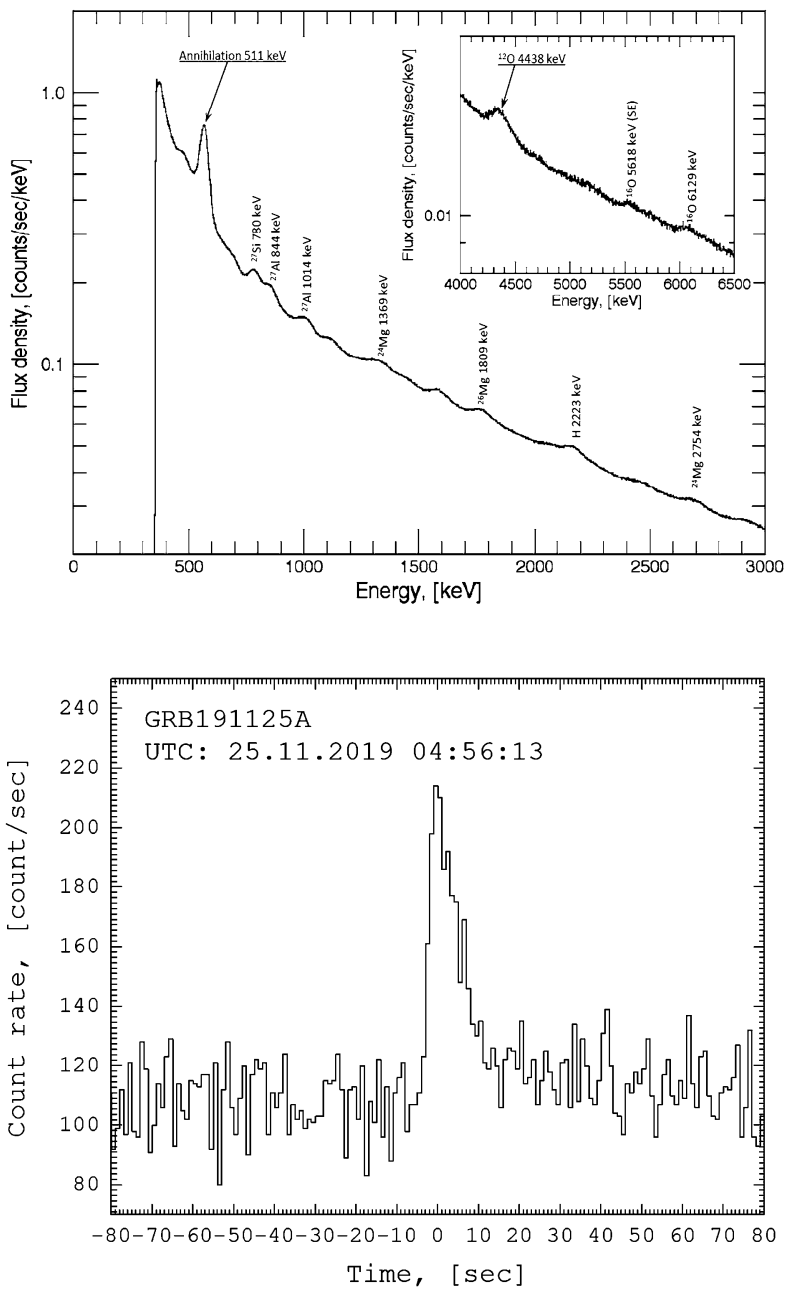

about 2-3 per month. An example of MGNS GRB measured with time resolution of $1 \mathrm{sec}$ is presented in Fig. 9.

Gamma-rays originating from solar flares are also detectable by MGNS. Solar flares are non-stationary and anisotropic processes, and the ability to observe them from different directions in the Solar System is crucial for further understanding of their development and propagation, as it has been demonstrated in the case of HEND instrument onboard Mars Odyssey (Livshits et al. 2017). Though MGNS has not detected any solar events during its first 13 months, as the solar activity will increase during the present Solar Cycle 25, many flares are expected to be detected.

The MGNS instrument will also perform special sessions of measurements during flybys of Earth, Venus, and Mercury with the objective to measure neutron and gamma-ray albedo of the upper atmosphere of Earth and Venus and of the surface of Mercury. Another objective is to test the computational model of the local background of the spacecraft using the data measured at different orbital phases of flyby trajectories. The low altitude flybys (such as the $551 \mathrm{~km}$ for 2nd Venus flyby and three $200 \mathrm{~km}$ flybys for Mercury) would be the 
Fig. 10 Case of

BepiColombo-Earth opposition. Trajectories of different spacecraft (BepiColombo, Solar Orbiter, Akatsuki (Venus), Parker Solar Probe) for 18 March 2021, plus/minus three months. The Sun is at the center, the location of Earth is fixed on the $+X$ axis (black asterisk)

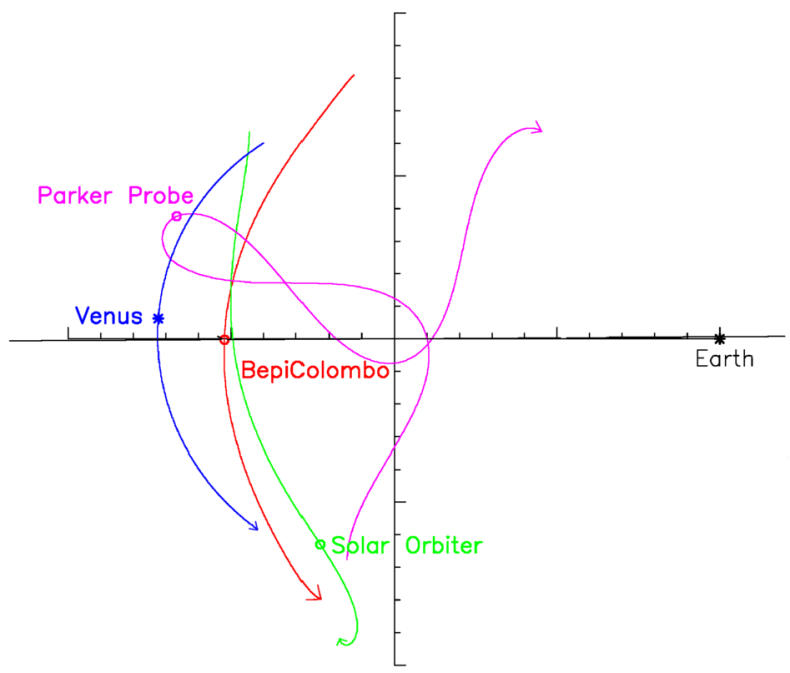

most useful for such tests, because the spacecraft would be shadowed from cosmic radiation at very different distances from the planet. Neutron and gamma-ray measurements during Earth flybys should also enable investigation of the interaction between solar wind and Earth environments as well as studies of spacecraft neutron and gamma-ray background upon its passage through the Earth's radiation belts.

\subsection{Methods}

\subsubsection{Multi-spacecraft Coordination}

The heliospheric investigations can greatly benefit of BepiColombo related observations during cruise, and the data achieved can improve the accuracy of the space weather predictions beyond the location of BepiColombo (e.g. at the Earth). As already pointed out, the journey of BepiColombo to Mercury and the operation around the Mercury orbit cover different portions of the solar cycle, starting from the late declining phase of cycle 24 , and up to all the rising phase of cycle 25 (see Fig. 3). In the intervening period between the April 2020 Earth flyby and Bepi Colombo's Mercury orbit insertion, several opportunities for heliospheric multi-point observations are possible via coordinated activity between BepiColombo and other active spacecraft, as well as with ground-based IPS observations. The opportunity to obtain measurements simultaneously at many different heliospheric locations inside $1 \mathrm{AU}$ is unprecedented (see Figs. 10, 11, and 12). Potential coordinated observations that may be planned would involve the following observation platforms:

- NASA's Parker Solar Probe (PSP) and ESA's Solar Orbiter (SolO) (launched in August 2018 and in February 2020, respectively, and that will be operating in the inner heliosphere for 7 years);

- JAXA's Akatsuki spacecraft orbiting around Venus since December 2015 (mission extension to 2024 presently under review);

- ESA's JUICE spacecraft (launch date June 2022), whose flight path includes an orbit around the Sun in the inner heliosphere prior to embarking on a direct trajectory to Jupiter in November 2026; 
Fig. 11 Plasma flow studies. Trajectories of different spacecraft (BepiColombo, Solar Orbiter, Akatsuki (Venus), Parker Solar Probe) for 10 August 2021, plus/minus three months. The Sun is in the center, the location of the Earth is fixed on the $+X$ axis (black asterisk), the arrow shows the direction of plasma flow

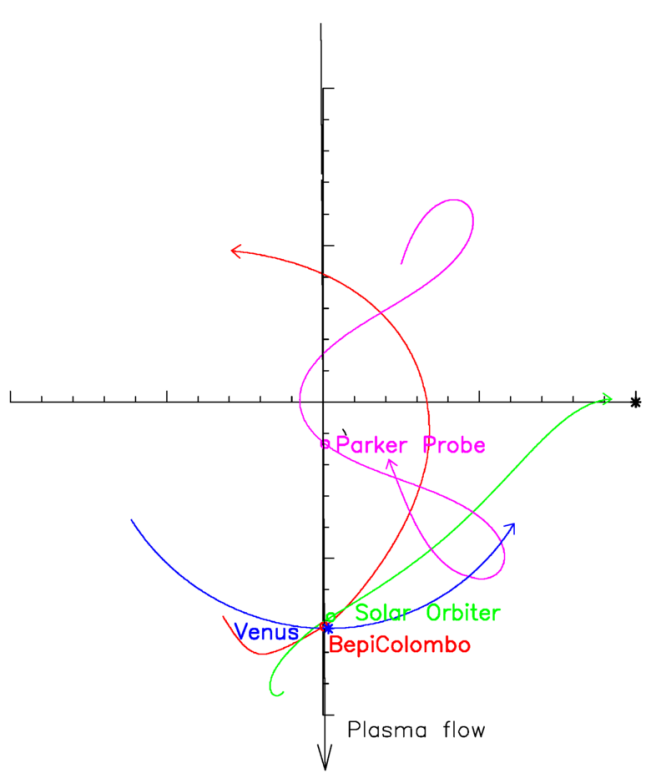

Fig. 12 A magnetic flux rope constellation of BepiColombo, Parker Solar Probe and Solar Orbiter on 18 March 2023. The Sun is in the center, the location of the Earth is fixed on the $+X$ axis (black asterisk). The black curved line shows the calculated Parker spiral of solar wind plasma with a velocity of $400 \mathrm{~km} / \mathrm{s}$

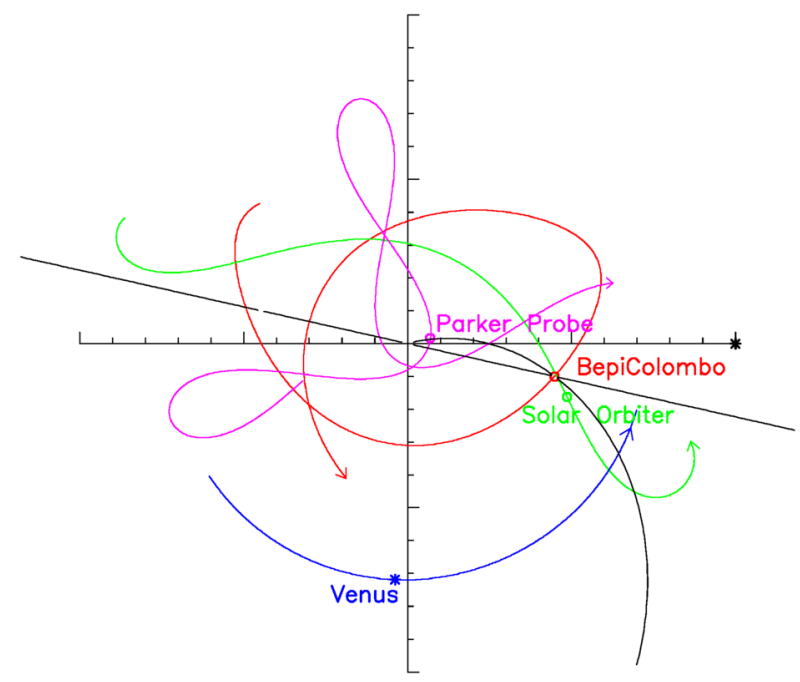

- NASA's Polarimeter to Unify the Corona and Heliosphere (PUNCH) spacecraft, to be launched in March 2023;

- NASA's STEREO A, which is an Earth orbiting, heliophysics observatory;

- Other Earth-orbiting spacecraft located at the L1 point such as SOHO, DSCOVR, ACE and Wind.

Below are the main interesting geometries of multi-spacecraft constellations to be analyzed:

- radial alignment of two or more spacecraft; 
- occultation, when two spacecraft lie on the opposite sides of the Sun, thus the heliographic longitude they face differ in $180^{\circ}$;

- magnetic alignment, when the spacecraft lie on the same Parker spiral (where the curve of the computed Parker spiral depends on the assumed, modelled or measured solar wind velocity).

For radial alignment studies, a distinction has to be done between two cases: (a) the alignment of several spacecraft at the same time (when each spacecraft is located on the same heliospheric longitude at the same time and facing the same heliographic longitude); and (b) the alignment that takes into account the plasma travel time (with retardation): in this latter case, the different spacecraft will be on the same heliospheric longitude at different times, hence they will have a chance to observe the same plasma parcel (provided that the solar wind velocity used to define the plasma travel time is accurate).

In order to understand solar wind acceleration, we need simultaneous observations of the solar surface, the corona, and the interplanetary solar wind. In addition to the in situ observations, the magnetic field line at the solar photosphere can be derived from high spatial resolution observations (i.e. by using Hinode/SOT Tsuneta et al. 2008). The global structure of the solar wind density and velocity in the inner heliosphere (0.2-1.0 AU) can be derived by the study of ground-based interplanetary scintillation (IPS) observations (e.g., Iwai et al. 2019), combined with global MHD simulations such as SUSANOO (Shiota et al. 2014), IPS-ENLIL (Jackson et al. 2015), or EUHFORIA (Pomoell and Poedts 2018) (see next Sect. 2.2.2). The solar wind structures obtained with these models, are combined with the in situ observations at the corresponding trajectory, in order to distinguish the time and spatial variations in the in situ data, and to understand background solar wind propagation characteristics (Fujiki et al. 2003). The ratio between Fe and H, the so-called First Ionization Potential (FIP) bias is used to determine the location of solar wind source based on the abundance of the elements. It can be measured by the PICAM and MIPA ion sensors of the SERENA package (Orsini et al., this journal) onboard MPO. The FIP bias at the low solar corona can be observed by EUV spectrometer onboard Hinode/EIS (Culhane et al. 2007) by its campaign observation modes. Therefore, we can find the origin of solar wind at the BepiColombo location by comparing the full disc mosaic observation of the FIP bias by Hinode/EIS and in-situ measurements by SERENA.

Heliospheric transients studies will greatly benefit from multi-spacecraft perspective. As discussed in detail above (Sect. 2.2.1), magnetic field observations from several space probes inside $1 \mathrm{AU}$ will be important to shed light on the propagation and evolution of ICMEs, their shocks and sheaths, SIRs and fast solar wind streams.

Magnetic field and plasma parameters observations will also be of help to characterize the evolution of plasma across the heliosphere and across different scales, thus advancing the investigating dynamical properties of solar wind evolution. Specifically, thanks to multispacecraft locations and observations (BepiColombo, SolO, PSP, ACE, Wind) several solar wind properties will be highlighted: the changes in the solar wind composition at different locations, how turbulent features evolve due to both solar sources and in situ processes, the role of thermal and suprathermal particle populations and their source mechanisms, and so on.

In addition to the local plasma and field measurements, STEREO A, PSP and SolO carry heliospheric imagers (Eyles et al. 2009; Vourlidas et al. 2016; Howard et al. 2019) that can be used to probe the density structures at the location of the inner heliospheric probes, including BepiColombo. Then, the ground-based IPS observations can be also used to derive the global density and velocity distributions of the inner heliosphere including CMEs (Iwai 
et al. 2019). MGNS, if operated during solar flares, can deliver important information on the gamma and neutron spectrum related to solar flares (see also Sect. 2.1.3).

The study of Solar Energetic Particle events can also benefit a lot from multi-point observations: one of the main advantages is the greatly enhanced capability to separate transport effects from the dynamics of the particle accelerator at the source, which is smeared to a great extent when particles are observed from 1-AU locations only (Desai and Giacalone 2016). Both the number of observing spacecraft and their varying radial and longitudinal separation will be an asset in disentangling the effects of source dynamics and transport. The SIXS-P instrument measuring energetic particles, if operated during the cruise phase and combined with energetic particle observations of PSP, SolO, and spacecraft at $1 \mathrm{AU}$, may help understanding of the transport and acceleration processes.

Figures 10, 11 and 12 show some of the above mentioned configurations for BepiColombo, Solar Orbiter, Akatsuki (orbiting around Venus) and Parker Solar Probe:

1) on 15-18 March 2021 BepiColombo and Akatsuki will be behind the solar corona (hidden from Earth) close to each other (Fig. 10). Radio waves transmitted from BepiColombo and Akatsuki can cross the solar corona almost simultaneously to allow multipoint measurements along the same diagonal. Parker Solar Probe will be close on the Eastern side and Solar Orbiter will be on the Western side of the two occulting spacecraft. In March 2021 solar activity will be low, therefore conditions in the corona can be expected to be more or less stable for a couple of days. Having Parker Solar Probe and Solar Orbiter at different longitudes makes it possible to investigate eventual fast streams or CMEs as they sweep across the different spacecraft. On 15 March BepiColombo and Akatsuki will be radially aligned, on the 16th they will be radially aligned with retardation, i.e. Akatsuki will observe the same plasma package that left BepiColombo on the 15th. On 18 March BepiColombo will be in opposition with Earth (Akatsuki very close) making it ideal for radio science.

2) The period around 10 August 2021 offers remarkable possibilities to coordinated measurements, for BepiColombo flyby Venus, 1 day after Solar Orbiter flyby at Venus too. The event is ideal for cross-calibration and eventual detection of magnetic holes around Venus (see Fig. 11 and Sect. 5). Having Parker Solar Probe also radially aligned makes it possible to have a 3-dimensional picture of solar wind (eventually fast streams, CMEs, $\mathrm{SEP}$ ) propagation. The plasma parcel observed by Parker Solar Probe on the 10th of August at the indicated time reaches BepiColombo on the 14th of August at 2 a.m. if its velocity is $400 \mathrm{~km} / \mathrm{s}$. The latitudinal difference between Parker Solar Probe and BepiColombo is small $\left(\sim 0.4^{\circ}\right)$ : thus, there is a good chance to observe exactly the same plasma and investigate its propagation. Furthermore, the latitudinal distance between the three spacecraft at Venus at $0.7 \mathrm{AU}$ is $\sim 1^{\circ}$. At a distance of $0.7 \mathrm{AU}$, this is enough to observe plasma expansion. Before the "triple meeting", Parker Solar Probe will be on the opposite, eastern side of the Sun on the 7th of August 2021. There is a chance for solar corona analysis with radio observations using the Parker Solar Probe and all three probes along the same radial.

3) One of the several opportunities for coordinated measurements of magnetically aligned spacecraft will occur on 18 March 2023, when Parker Solar Probe, BepiColombo and Solar Orbiter will be very close to the same Parker field line (Fig. 12). Calculations were made by assuming solar wind velocity to be $400 \mathrm{~km} / \mathrm{s}$, but a time window can be defined using different velocity values. If the probes are also close in latitude, they can study the changes of the solar wind source region. In the case shown, Solar Orbiter, Parker Solar Probe, and BepiColombo are wider apart in latitude: they will be located at 0,6 and $12^{\circ}$ from the Ecliptic. With this constellation one can study the 3-D variability of solar wind 
plasma. This will also offer the unique opportunity to investigate the spatial evolution of solar wind turbulence properties as well as to provide a deeper understanding of the nature of the intermittency, continuously debated between a temporal and a spatial phenomenon. Moreover, this will be helpful for testing Taylor frozen-turbulence hypothesis and investigating the role of non-stationarities (Taylor 1938; Alberti et al. 2020; Chen et al. 2020).

The possible multi-spacecraft constellations are not limited to the above cited cases; several further special geometries (e.g. quadrature, widely spaced) can be considered where the different latitudinal position of the different spacecraft may offer a 3-dimensional investigation of solar wind properties. On the other hand, before proposing a coordinated observation campaign, a series of constraints have to be further checked, e.g. the operability of the instrument, its field of view, position, etc.

\subsubsection{Support of Space Weather Modeling}

Important support to multi-spacecraft measurements may come from the space weather simulation tools presently available in the scientific community.

In particular, the model predictions of solar wind propagation and interaction with the planetary environments may be used to support the interpretation of the measurements performed during the cruise (both far from the planetary environments, and close to planetary flybys). On the other hand, the BepiColombo measurements will provide precious data to verify and improve current space weather models and prediction tools.

Subsequent detailed analysis on the ground of the data provided by active MPO and Mio instrumentation will overall allow a database of snapshots of the solar wind environment at different distances from the planet to be built up and to, thereby, deduce how Venus and Mercury interacts over time with the extant solar wind at a range of distances from its surface, thus yielding important insights into how different kinds of solar wind stimulate different planetary responses.

Several state-of-the-art models/tools are available to provide space weather predictions at the BepiColombo spacecraft:

1 the Interplanetary Scintillation 3D-reconstruction Technique (IPS analyses) which provides precise tomographic 3-D reconstructions of the time-varying global heliosphere (Jackson et al. 2011, 2015). This methodology incorporates both the background solar wind and ICMEs and iterates to provide the best boundary values of velocity and density that are present globally, as well as measured in-situ over the viewed volume. Also, it extrapolates magnetic fields from the solar surface to this same inner boundary.

2 ENLIL (Odstrcil and Pizzo 1999; Odstrcil et al. 2005; Odstrcil 2003), is a time-dependent 3-D MHD model of the heliosphere which solves equations for plasma mass, momentum, energy density, and magnetic field using the Total-Variation-Diminishing Lax-Friedrichs (TVDLF) algorithm. The standard way ENLIL is operated uses magnetic field measurements from the solar surface to provide a quasi-stationary solar wind model with velocity and density boundary input parameters derived from the Wang-Sheely-Arge/WSA Potential Field Source Surface (PFSS) model (Arge and Pizzo 2000). Injected into this background are "cone" CME inputs (e.g., Luhmann et al. 2010) from LASCO coronagraph data. ENLIL supports mass, and mass flux conservation, heating terms over solar distance, and non-radial transport of structures from its inner boundary, which is usually set at $0.1 \mathrm{AU}$. 
An upgraded analysis system to be utilized at Venus during the flybys melds the first two systems described above together, thereby allowing the IPS data to iteratively update and fit ENLIL modelling so as to ultimately provide a rapid forecast of Coronal Mass Ejections and shocks, as well as of CIRs at inner heliospheric planets using the ENLIL 3-D MHD model as a kernel. This can provide all the accoutrements of the ENLIL system, and/or IPS tomography and imagery. In addition, the combined system updates this modelling as the solar wind flows outward from the ENLIL inner boundary. The combined system can trace the trajectories of interplanetary magnetic field lines in 3-D, thereby enabling simulations of the magnetic connections from locations on the Sun to BepiColombo and to other contemporaneously flying spacecraft (such as SOHO, ACE, and STEREO-A), as well as the Parker Solar Probe which monitors ambient energetic solar particles.

Other available space weather simulation tools could be put in use in a similar way to provide a useful forecast of relevant space weather effects at BepiColombo position:

- The European heliospheric forecasting information asset (EUHFORIA - Pomoell and Poedts 2018) is a physics-based simulation model of the inner heliosphere driven by boundary conditions based on empirical models. This simulation tool has been specifically designed for space weather forecasting purposes. EUHFORIA consists of two components: a coronal model and a heliosphere model including coronal mass ejections. The coronal model reconstructs a large scale model of the coronal magnetic field and make use of empirical relations to determine the plasma state (solar wind speed and mass density). These quantities are then used as boundary conditions to drive a 3-D time-dependent magnetohydrodynamics model of the inner heliosphere. CMEs are injected into the ambient solar wind modeled by using the cone model, with their parameters obtained from fits to imaging observations. Upcoming improvement of EUHFORIA will take into account both the CME internal magnetic field and a time-evolving solar wind.

- In the last years, lightweight, fast semi-empirical or physics-based models have been used to implement an ensemble modeling of the CME and solar wind transient propagation. Ensemble modeling incorporates the intrinsic limitation of information due to measure errors and lack of knowledge in the form of probability distributions. In practice, instead of a single run to forecast an ICME propagation, a set of runs, driven with input parameters extracted from suitable distributions are used to retrieve a distribution of output parameters. Among those models, the P-DBM (Napoletano et al. 2018) and the DBEM (Dumbović et al. 2018) can run thousands of single simulations in seconds and thus explore thoroughly the parameter space. The model outputs are the most probable ICME travel time and velocity at a heliospheric position, as well as the associated prediction uncertainties.

\section{Radio Science}

\subsection{MORE: The Mercury Orbiter Radio Science Experiment}

Radio science experiment, as already cited, is the only originally planned science activity during the cruise of BepiColombo. Superior Solar Conjunctions (SSC) can be used to carry out tests of general relativity, similar to those previously performed with the Viking and Cassini missions (Reasenberg et al. 1979; Bertotti et al. 2003). In the Viking experiment the spacetime curvature generated by the mass of the Sun (controlled by the postNewtonian parameter $\gamma$, equal to 1 in General Relativity) was derived from measurements 
of the time delay of radio signals sent by a ground station and coherently returned to Earth by means of an onboard transponder. The observable quantity in the Cassini determination was the frequency shift of the carrier (proportional to the spacecraft range rate). The use of a multi-frequency link at $\mathrm{X}$ and $\mathrm{Ka}$ band $(7.2-8.4 \mathrm{GHz}$ and $32.5-34.0 \mathrm{GHz}$ ) allowed a nearly complete cancellation of the noise due to the interplanetary and coronal plasma and an improvement by a factor of 50 of the Viking results (Bertotti et al. 1993, 2003).

The Viking experiment was limited by the lower frequency of the radio link, at 2.1$2.3 \mathrm{GHz}$. Now the cruise tests to be performed by MORE on BepiColombo will combine for the first time range and range rate observables, and a plasma noise cancellation system based on the use of multiple frequencies. The Mercury Orbiter Radio Science Experiment (MORE) is based on the use of two onboard transponders: a dedicated radio science Ka band transponder (KaT), supporting a coherent link at Ka band (both uplink and downlink), and a TT\&C deep space transponder (DST) supporting an X band uplink (7.2 GHz) and a dual frequency, coherent $\mathrm{X}$ and $\mathrm{Ka}$ band downlink at 8.4 and $32.5 \mathrm{GHz}$, respectively (for a complete description of MORE, see Iess et al., this journal).

MORE has two additional science goals for the cruise phase:

- the advanced radio system, complemented by data from the ISA accelerometer, can potentially provide significant improvements in the determination of the spacecraft trajectory.

- a comparison between the standard navigation system and the augmented system available for BepiColombo's geodesy and relativity experiments (especially the $\mathrm{Ka} / \mathrm{Ka}$ link) can pave the way for its adoption in spacecraft operations.

Quantifying these improvements in spacecraft navigation is a primary goal of the MORE team. The spacecraft was tracked also during the Earth flyby (April 10th, 2020), and collected data that will be used to possibly better model the spacecraft trajectory orbit and improve the orbit determination codes.

In addition, MORE may contribute to studies on solar corona heating and on acceleration of the solar wind that are important topics of solar and heliospheric physics. The key processes are propagation and dissipation of the magnetic energy between the solar surface and the outer corona. MORE will produce a wealth of plasma calibration data during the SSC used for testing relativistic gravity. These data include the uplink and downlink Total Electron Content (TEC) and their variation with time, as shown by Bertotti et al. (1993, 2003). Uplink and downlink TEC and its time derivative are inferred from a linear combination of, respectively, range and range rate measurements in the three radio links (X/X, X/Ka and $\mathrm{Ka} / \mathrm{Ka}$ ). The ability to separate the uplink and downlink TEC along with its variations was never possible before and it is peculiar to BepiColombo. In the geometric optics limit, TEC is proportional to the integral of the refractive index along the line of sight, which, in turn, is proportional to the plasma density at microwave frequencies. (Magnetic corrections to the refractive index will likely not produce detectable effects after 2-3 solar radii.) The data could therefore be exploited not only to probe the density of the solar corona down to a few solar radii (a method often exploited in the past, see, for example, Miyamoto et al. 2014, with earlier references therein) but also for correlative analyses with the plasma instruments onboard BepiColombo and other spacecraft (such as ESA's Solar Orbiter and JAXA/Akatsuki), as well as observations from ground and near-Earth satellites. The separate determination of the uplink and downlink plasma lends itself to the space-time localization of large plasma events (such as CME, or CIRs) along the line of sight, thus complementing the information provided by imaging of the solar corona (Richie-Halford et al. 2009). In addition, the tracking data acquired during SSC may contain a contribution from the coronal magnetohydrodynamic waves and, in principle, provide also the solar wind velocity (via a time delay in the phase fluctuations at $\mathrm{X}$ and $\mathrm{Ka}$ band due to the differential bending of the two radio waves). 


\subsection{ISA: Measurements of Non-gravitational Accelerations}

The measurements of MORE will be complemented by the Italian Spring Accelerometer (ISA) (Iafolla and Nozzoli 2001; Iafolla et al. 2007, 2010, 2016; Lucchesi and Iafolla 2006, and Santoli et al., this journal) by providing measurements of the non-gravitational perturbations (NGP) acting on the MPO spacecraft. These perturbations are due to surface forces, like direct solar radiation pressure, that carry away by a small amount the spacecraft trajectory from a purely gravitational (geodetic) one. ISA is a three-axis instrument, i.e. it provides (once its data are properly calibrated and reduced) the full three-dimensional vector representing the overall non-gravitational acceleration acting on the spacecraft. The instrument has been designed to operate on a wide signal frequency band $\left(3 \cdot 10^{-5}-10^{-1} \mathrm{~Hz}\right)$.

BepiColombo marks the first time that a high-sensitivity accelerometer - fully dedicated to scientific measurements - is embarked on a deep-space mission. Accelerometers with similar performance have been employed just on Earth geophysics missions, such as CHAMP (Reigber et al. 2006), GRACE (Tapley et al. 2013), GRACE-FO (Kornfeld et al. 2019), and GOCE (Drinkwater et al. 2006). It has to be noticed that, by construction, the accelerometer is able to sense both NGP due to external surface forces and internally generated signals (e.g. micro-vibrations). ISA data can be used as well to monitor the platform behaviour in terms of vibrations and rotations produced by antennas, mechanisms, solar panels, reaction wheels, etc. A non-exhaustive list of measurements that could be performed at selected times in the cruise includes:

- NGP acting on the MCS;

- planet-induced gravitational gradients during flybys;

- NGP during superior solar conjunctions;

- MCS accelerations along the thrust-no thrust transitions;

- density changes within the magnetosphere (boundary regions) during flybys;

- detection of micrometeoroid and dust impacts.

The measurement of non-gravitational effects in various cruise phases would be an interesting result by itself, besides being a direct verification of the instrument capabilities before the nominal in-orbit phase. Furthermore, the SSC will feature the full tracking capabilities of BepiColombo, opening an interesting calibration opportunity for ISA. Providing an independent measurement of the transition from thrust to no thrust orbit arcs could be useful in order to better assess the solar electric propulsion performance.

The possibility of measuring gravitational gradients on the spacecraft in the flyby phases deserves special attention. Due to design constraints, ISA has not been placed in the MPO center of mass; therefore a (small) gravitational gradient by the planet arises between the center of mass and the position of ISA proof masses. Hence, it is expected that during flyby with relatively low altitudes at closest approach, where the contribution is higher, the gravitational gradient of the planet will be part of the signals measured by the accelerometer. While this could have been an issue in the nominal orbital phase (expected to be solved by the use of the so-called Schulte vector), it will provide a potential calibration signal during the flybys. With the ever-increasing interest in gravitational gradiometers as instruments for the direct measurement of the gravitational field of Solar System bodies, where accelerometers are coupled together to detect the gravitational gradient, such a measurement can be considered as a direct test of ISA potentiality in being a basic element of a space gradiometer.

A measurement to be potentially performed by ISA is the identification and possible characterisation of the transition regions within the planet magnetospheres during flybys. 
Crossing the different magnetospheric regions (like bow shock, magnetosheath, magnetopause, etc.) related to the interaction between solar wind and planetary magnetic field, means to travel through areas characterized by fluctuations in the density of particles and ions. Possible changes due to drag from charged particles (Milani et al. 1987) could be identified during flyby phases in the magnetosphere, specifically during the crossing of the magnetopause, i.e. the outer boundary of the magnetosphere. Such an opportunity is strongly dependent on the flyby geometry with respect to the magnetosphere structure and on the encountered density changes. Moreover, solar activity (solar wind, CME, 11-year cycle) deeply affects the magnetosphere shape and the particle densities.

To provide effectiveness to this approach, ISA measurements will be correlated with data from other switched-on instruments during the flyby and providing information on magnetic field variation, plasma measurements, particles energy/density/composition derived from other onboard instrumentation, such as MPO/MAG, SIXS-P, BERM, MIPA, PICAM, PHEBUS, and all Mio sensors. Collection of data from analogous instruments onboard other spacecraft monitoring the Earth environment during the flyby would be beneficial to the observations.

A further possibility for ISA science is to corroborate possible detection of micrometeoroids and dust impacts on the spacecraft. This method has been already proved with MMS (Magnetospheric Multiscale Mission) spacecraft (Williams et al. 2016); in the present case, ISA observations should be carried out together with the Mio/MDM instrument, which is devoted to dust particles detection but suffers limited field of view (see Sect. 2.1.2).

Finally, the cruise phase is an additional opportunity to directly test and validate the instrument performance of ISA in a relatively quiet environment. Indeed, it is expected to be much quieter than the orbital phase for a number of reasons (i.e., greater distance from the Sun, reduced on-board activity, and very stable attitude). Measuring the background noise in selected cruise periods will be useful to fine tune the instrument error model and to characterize its long-term behavior.

\section{Earth Flyby}

The first BepiColombo flyby at the Earth was needed to deflect the spacecraft into the inner Solar System, and towards the orbit of Venus. The Earth flyby occurred on the 10th of April 2020 with a closest approach (CA) at 04:25 UTC, and an altitude of $12684 \mathrm{~km}$.

The maximum apparent size was $37^{\circ}$ for the Earth, and $\sim 0.5^{\circ}$ for the Moon. The geometry of the flyby and of the relative positions of MCS, Earth and Moon are depicted in Fig. 13 (upper panels), together with the average position of the Earth's plasma boundaries (bow shock and magnetopause) crossed by the spacecraft, with relative time in UTC; in the bottom panel the variation of altitude and of the Sun-MCS-Earth and Sun-Earth-MCS angles are also shown.

As discussed in Sect. 1, during interplanetary cruise and excluding electric propulsion phases, the default spacecraft attitude has the $+Y$ axis directed towards the Sun. The need for angular momentum load minimization was relaxed for closest approach \pm 1 day, corresponding to the period of scientific interest. The general attitude constraints shown in Table 9 (in the Appendix) apply here: at a Sun distance of 1 AU, it is possible to offset the Sun direction in the spacecraft composite $+Y Z$ plan in the range between +47 and $-9.1^{\circ}$. A roll phase around the Sun direction is also possible (360 rotation). According to attitude constraints and instrument requests, MCS operated in a quasi-inertial attitude with the Sun along $+Y$ and with a phase of $250^{\circ}$. 

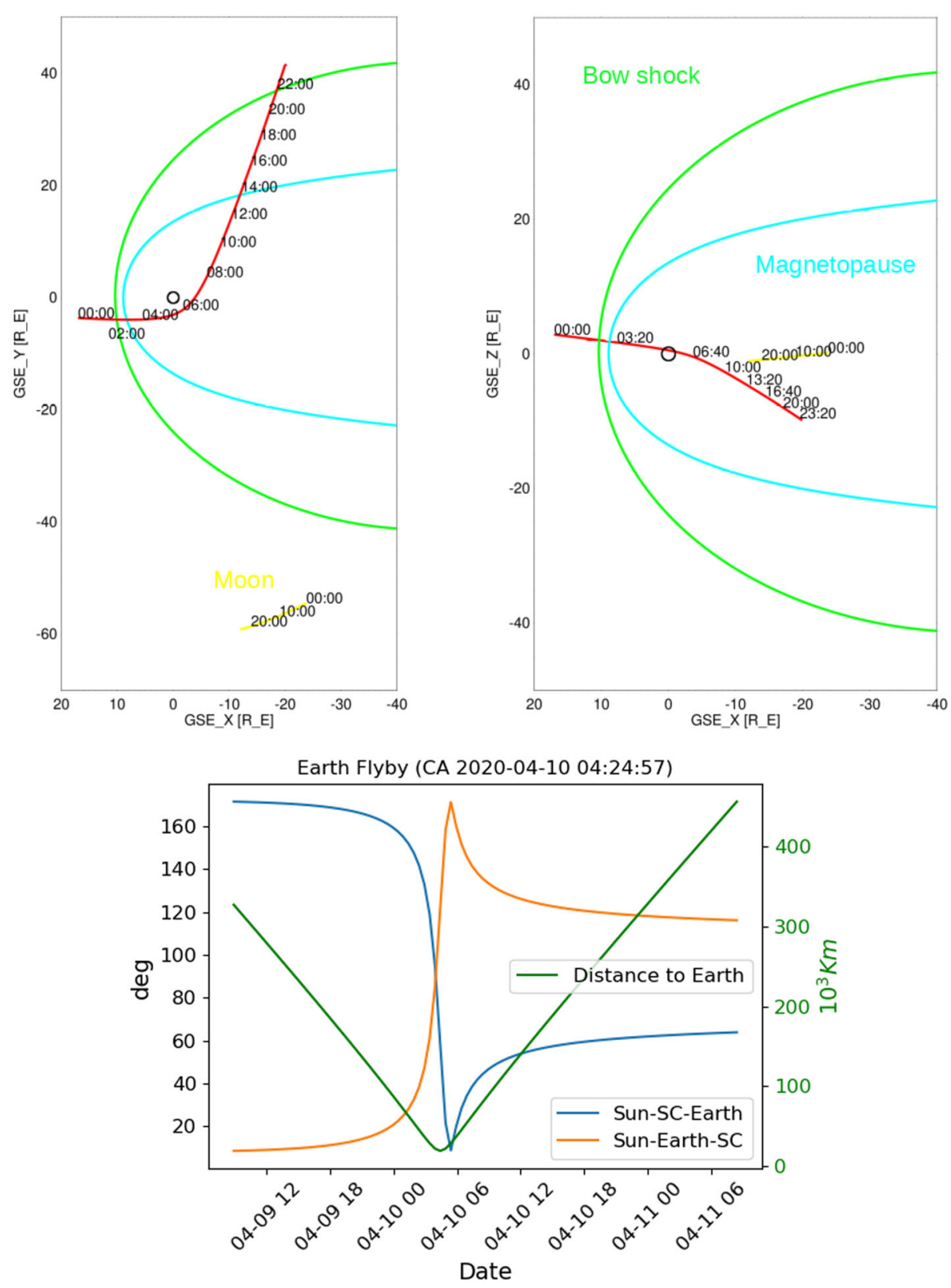

Fig. 13 Upper panels: BepiColombo trajectory on a Geocentric Solar Ecliptic System (GSE) $X-Y$ plane (to the left) and GSE $X-Y$ plane (to the right). Bow shock and magnetopause average positions are drawn in green and cyan respectively. In red the trajectory. Moon position is also shown in yellow. Lower panel: Linear and angular distances (Sun-MCS-Earth and Sun-Earth-MCS) of BepiColombo to the Earth's center in the 48 hours around closest approach 
Moreover, power flux density constraints implied a switch from HGA to MGA to LGA respectively 7, 2, and 1 days before (and after) closest approach. 34 minutes of solar eclipse occurred at $\mathrm{CA}+36$ minutes (the maximum duration allowed, as driven by the capacity of the MTM battery). During eclipses, the link between MTM and MPO was turned off, so each module is provided with its own power battery to be charged to $100 \%$.

\subsection{Scientific Objectives}

The Earth flyby was the first opportunity to operate several instruments of BepiColombo at the same time. The instrument operations were mainly for calibration purposes, to observe the well known environments of the Earth, and the Earth-Moon system. The geometry of the flyby (see Fig. 13) offered a very close approach to Earth surface $\left(<2 R_{E}\right)$, while the Moon was farther away from the spacecraft in the opposite direction $(>300.000 \mathrm{~km})$. The Moon is often used as a calibration target due to its well-known flux. Those calibration observations may sometimes result in major scientific discoveries, as happened in the past for the temporal and spatial variability of Moon surface hydration rate as observed by Deep Impact (Sunshine et al. 2009), or the detection of adsorbed water and hydroxyl on the Moon by Cassini (Clark 2009), and Chandrayaan-1 (Pieters et al. 2009).

Both MERTIS and PHEBUS observed the Moon, before and after the CA respectively. Both instruments required pointing. MERTIS obtained the first hyperspectral data ever of the Moon in the thermal infrared from space with a spatial scale of $500 \mathrm{~km}$. Apart from using this type of observation for validating the calibration of the instrument, MERTIS measurements provided a new science dataset of the Moon surface composition and a baseline for comparison with the future Mercury datasets (i.e. the two celestial bodies, Moon and Mercury, being very similar in terms of surface appearance and exosphere). PHEBUS also observed the Moon, in two time-slots after the CA with the purpose of checking the absolute calibration of both EUV and FUV channels and characterize the pixel to pixel sensitivity of the two detectors (e.g. flatfield correction). These data will be compared to previous observations of the Moon in the UV range.

Regarding the measurements of the Earth environment, interesting data were acquired related to the crossing of the different regions of the planetary magnetic field (see Fig. 14).

The MPO-MAG started high rate observations $(128 \mathrm{~Hz}) 33$ hours before CA which allowed observing the interplanetary magnetic field (IMF) while outside the Earth's bow shock and the terrestrial magnetosphere after crossing the magnetopause. In total, the spacecraft spent 14.5 hours within the magnetospheric cavity. These measurements are of great importance to check the absolute sensor orientation. Simultaneous measurements of the magnetometer MGF onboard Mio, as well of other magnetometers onboard other spacecraft orbiting around the Earth (Cluster, Themis, MMS) have been acquired. In particular, MGF used the data of the solar wind for instrument offset determination, and the Earth magnetic field for inter-sensor alignment corrections.

PICAM and MIPA sensors from the SERENA package acquired measurements consistent with the ones observed by MPO-MAG. In particular, different ion populations have been identified with different energy levels and counts when crossing the magnetic boundaries, the outer radiation belts, the plasmasheet, and the low latitude boundary layers. The two SIXS detectors measured the proton and electron profiles well before and after the closest approach, including the range $\mathrm{CA} \pm 7$ hours, the near-Earth solar wind, the Earth's foreshock region, and the magnetosheath; and used them to cross-calibrate the detector elements with each other. In addition, by simultaneously observing with the X-ray detection system, SIXS calibrated the X-ray sensor with the particle background. 


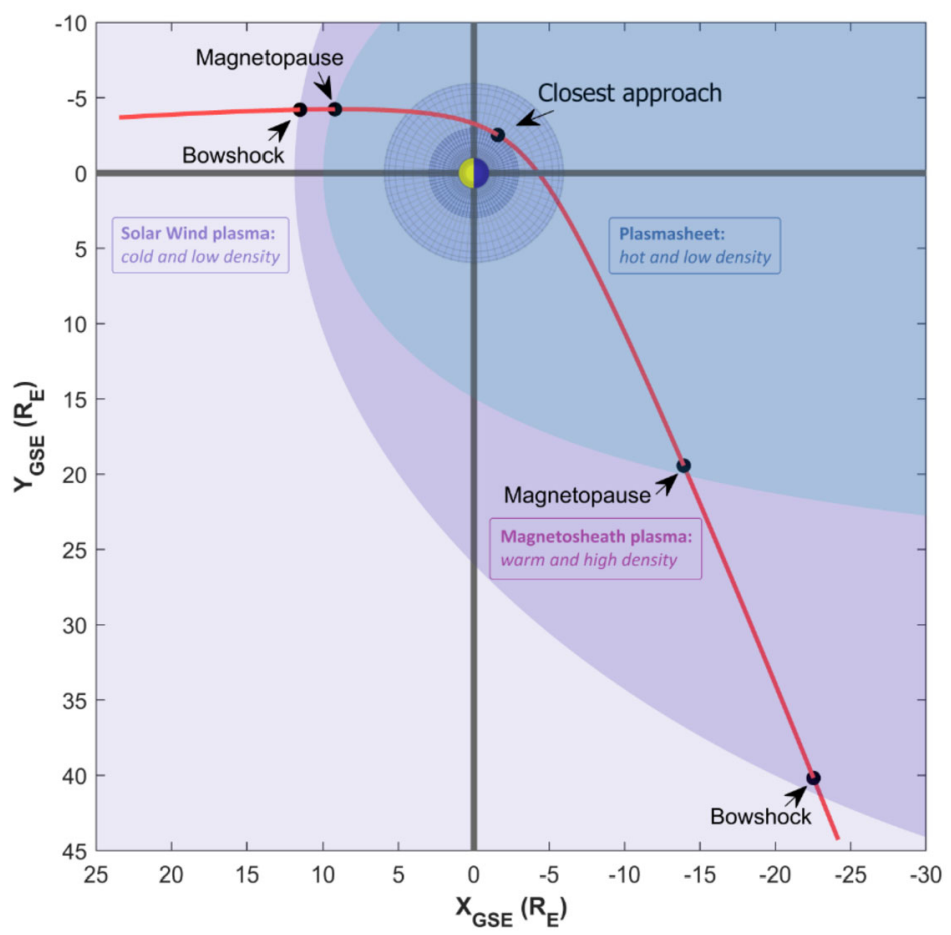

Fig. 14 Projection of the BepiColombo trajectory on GSE frame, $X-Y$ plane. The blue area highlights the Earth's magnetosphere, and the violet area the magnetosheath. The trajectory of the BepiColombo (red line) crosses the magnetopause and bow shock (Shue et al. 1998; Jeřáb et al. 2005). The two mesh-circles around the Earth represent the average location of inner and outer radiation belts. The Moon is not shown because outside of the figure box

Measurements by the accelerometer ISA attempted to detect the variation of the charged drag on the MCS due to the variation of particle density (see Sect. 3). Also instruments onboard Mio had a unique opportunity to monitor the Earth magnetosphere, plasmasphere, and radiation belts, with relevant observations for calibration and sampling purposes. During the Earth flyby period, the plasma particles by MPPE-MSA, MEA, MIA, HEP-ele, and ENA, the magnetic fields by MGF, and the magnetic waves by PWI have been observed.

In addition, the MGNS detectors were on, with the goal to measure neutron and gammaray albedos of the upper atmosphere of the Earth, as well as background signal during the passage through the radiation belts near the phase of closest approach (see Sect. 2.1.3). The data from Earth flyby will help to properly interpret MGNS data at Mercury, and the effects of Mercury's magnetosphere over the MGNS measurements.

Finally, the geometry of the flyby provided favorable conditions for exploring spacecraft orbit determination of ingress and egress arcs. In fact, current orbit determination algorithms do not treat ingress and egress arcs with sufficient precision to reconcile the predicted and measured delta-v: this difference is often mentioned in the literature as the "Earth flyby anomaly" (Turyshev et al. 2009).

The joint measurements of MORE and ISA may reduce the uncertainty on the measured delta- $\mathrm{v}$ and, help to better understand the origin of this discrepancy. 
Table 2 Venus flybys dates and their main characteristics. No eclipse and no occultation occur in both cases. Electric propulsion will not be used close to first flyby (then, classified as N/A in the table)

\begin{tabular}{lll}
\hline & Venus flyby 1 & Venus flyby 2 \\
\hline Date & 15 October 2020 & 10 August 2021 \\
Time & $03: 58: 31$ UTC & $14: 00$ UTC \\
Closest Approach altitude (km) & 10721.6 & $\sim 550$ \\
Venus-Sun distance (AU) & 0.72 & 0.72 \\
Venus-Earth distance (AU) & 1.17 & 1.25 \\
Sun-Earth-MCS angle (deg) & 38 & 25 \\
Electric propulsion end before CA & N/A & $\mathrm{CA}-30 \mathrm{~d}$ \\
Electric propulsion start after CA & N/A & $\mathrm{CA}+7 \mathrm{~d}$ \\
\hline
\end{tabular}

\section{Venus Flybys}

After the Earth flyby, the cruise to Mercury includes two consecutive flybys at Venus to reduce the perihelion to nearly Mercury distance and place the spacecraft in the same orbital plane as Mercury. The flybys take place on October 15th, 2020 and August 10th, 2021, and their main characteristics are summarized in Table 2. Figure 15 shows the flyby's trajectories in the Venus Solar Orbital (VSO) frame where the $+X$-axis points towards the Sun (left), the $+Y$-axis against the Venus orbital velocity vector and the $+Z$-axis northward. The map in the background shows expected ion mass fluxes and green and cyan lines show average positions of the bow shock and ion composition boundary layer (after Martinecz et al. 2009). Figure 16 provides the flybys linear and angular distances of the MCS, during the ingress and egress phases.

The two flybys are quite different both in terms of altitude and trajectory. During the first flyby the MCS approaches Venus coming from the dayside evening flank, moving towards the tail of the induced magnetic field, and crossing both the bow shock and the ionopause, with the closest approach altitude being above $10000 \mathrm{~km}$. Conversely, during the second flyby the MCS will approach Venus down to about $550 \mathrm{~km}$ above Venus surface, and will come from the opposite direction, from night to dayside, but still passing close to the evening side (see Fig. 15).

In particular, the lower minimum altitude of the second Venus flyby will allow the MCS to fly over the sunlit part of Venus, providing Venus visibility to instruments with a field of view on $-Y$. The general attitude constraints, at a Sun distance of $0.7 \mathrm{AU}$, allow an offset of the Sun direction in the range of +27 to $-9.1^{\circ}$ in the spacecraft composite $+Y Z$ plane (see Table 9 in the Appendix). Present configurations of both Venus flybys are eclipse free, therefore no power constraints will be necessary in this case. In addition, no Earth occultation by Venus will occur in both cases though, depending on the selected attitude profile, possible blockage of the antennae coverage by the spacecraft may occur.

\subsection{Scientific Objectives}

At the beginning of the space travel era, Venus was one of the most visited planets by space probes. In the last two decades, only two missions were launched to orbit around Venus, though long lived and fully successful: the ESA mission Venus Express (2006-2014), and the JAXA-Akatsuki spacecraft (2015-active). Nevertheless, there are still many open issues related to this planet that require dedicated investigations, and regular monitoring. In addition, Venus is becoming increasingly important in the rapidly growing field of exoplanet science (Kane et al. 2019; Dong et al. 2020) because exo-Venus analogs may be common 

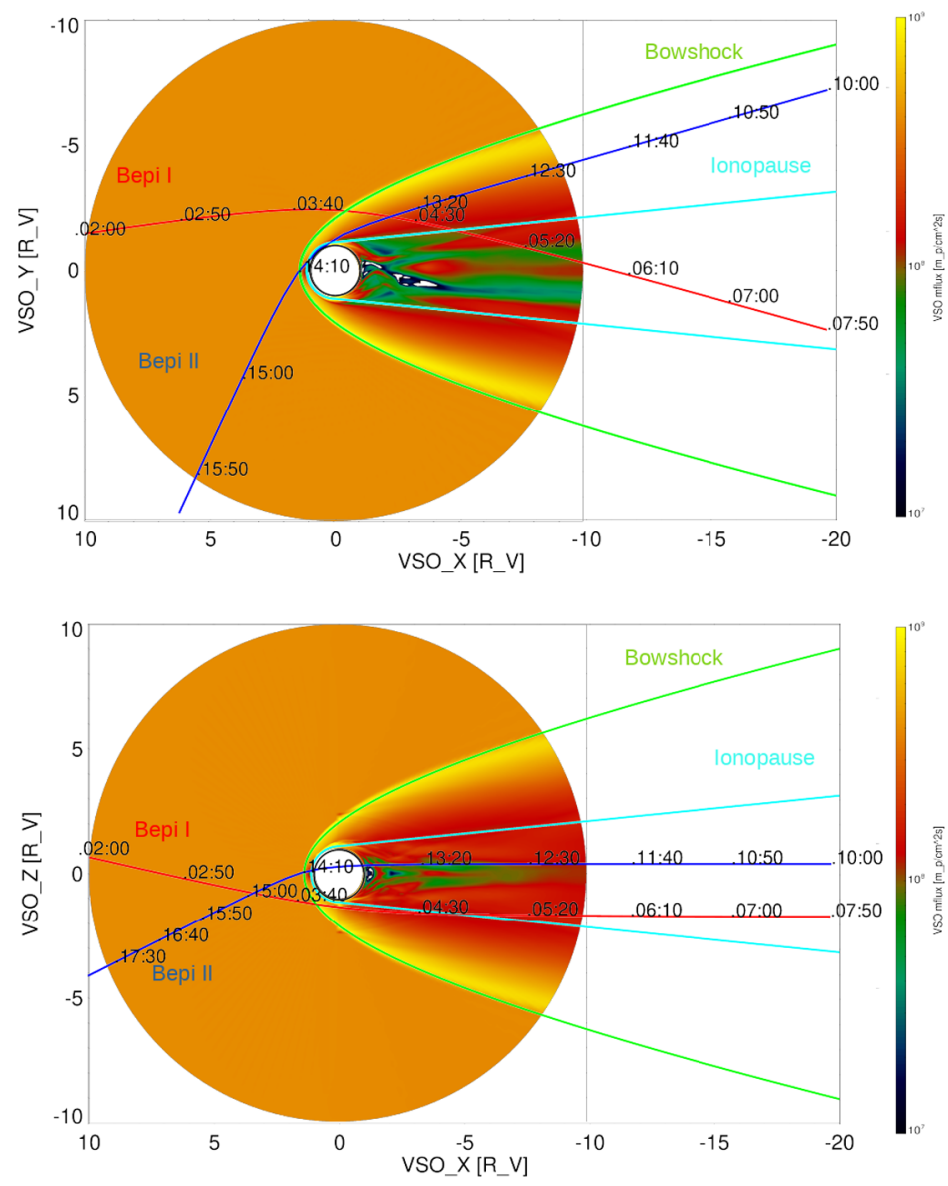

Fig. 15 The flyby trajectories of BepiColombo at Venus on October 15th, 2020 (Bepi I, red) and August 10th, 2021 (Bepi II, blue) projected onto the VSO $X-Y$ (top) and $X-Z$ (bottom) planes. Numbers plotted along the orbits give the expected universal time. Green and cyan lines show average positions of the bow shock and ion composition boundary after Martinecz et al. (2009) The color map in the background shows the expected ion mass fluxes in $\left[\mathrm{amu} /\left(\mathrm{cm}^{2} \mathrm{~s}\right)\right]$ from a 3D MHD model with spherical grids based on average solar wind and ionospheric parameters using the PLUTO code (Mignone et al. 2012)

types of planets and the near-future exoplanet detection and testing characterization methods at Venus is important to help future detections.

Hence, while waiting for a devoted mission to Venus, the frequent flybys of other spacecraft at Venus, necessary for the optimization of the orbits, are good opportunities to take additional data of the Venus environment (in terms of atmospheric measurements of composition, temperature, and wind vertical profiles, as well as of neutral and ionized particles, magnetic field, dust particles, etc.).

\subsubsection{Atmosphere}

Composition and Thermal Structure Key facts about the atmosphere of Venus were consolidated after the first exploratory phase carried out by Venera and Pioneer programs. 

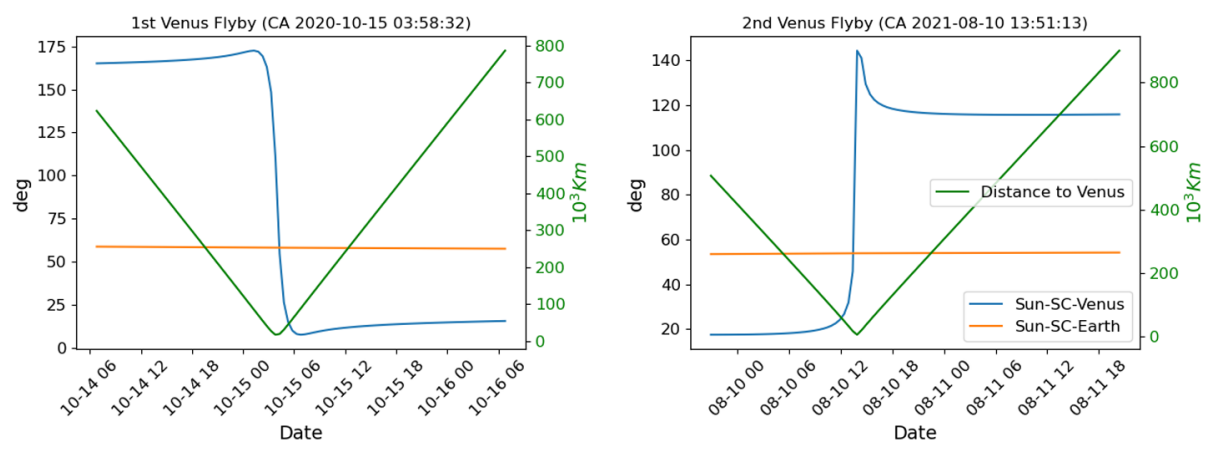

Fig. 16 First and second Venus flybys linear and angular distances of the spacecraft: to the planet (green), to Sun and Venus (blue), to Sun and Earth (orange)

Fig. 17 Example of air temperature profile in the Venus atmosphere, as derived from radio occultation experiment onboard Venus Express (from Limaye et al. 2018, after Tellmann et al. 2009). The tropopause is the approximate location of the main cloud decks

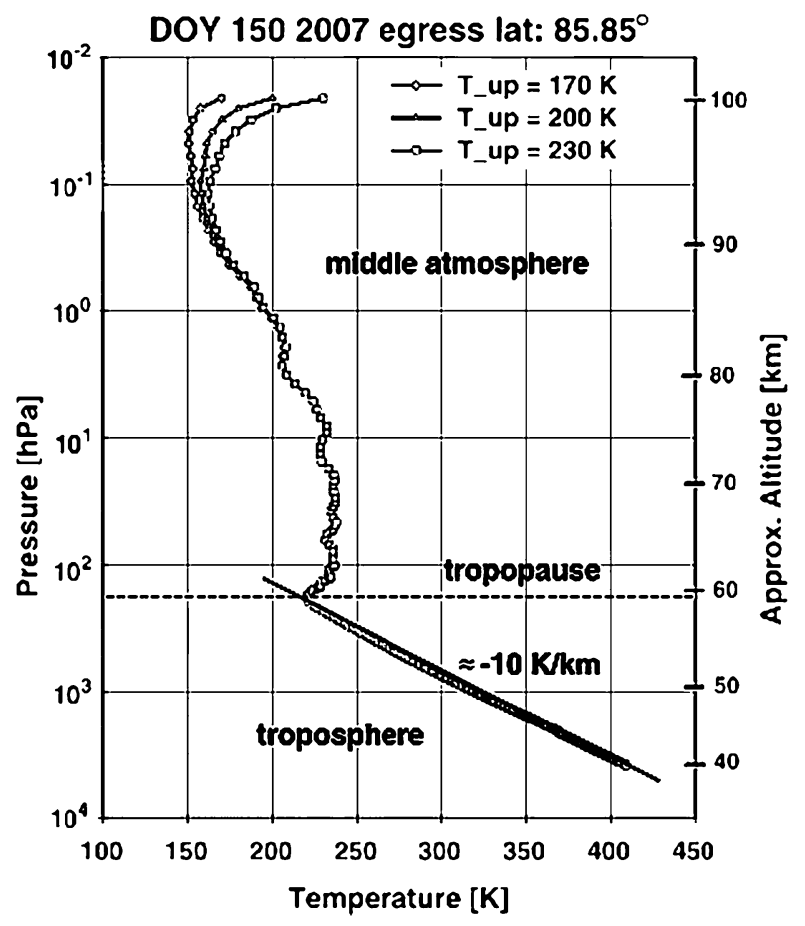

The thick atmosphere of Venus is $10^{-4}$ the mass of the planet, much higher than in the case of Earth and Mars. The surface temperature is $735 \mathrm{~K}$, and the pressure is 92 bars. The most abundant atmospheric gas is $\mathrm{CO}_{2}$ plus several percent $\mathrm{N}_{2}$ and other gases (notably, $\mathrm{SO}_{2}$ and $\mathrm{CO}$ ) as minor constituents. The water abundance is very low (between 1 and $15 \mathrm{ppm}$ at the cloud top, Cottini et al. 2015). The vertical structure of the Venus atmosphere is presented in Fig. 17: within the height range of $0-55 \mathrm{~km}$, the temperature lapse rate is nearly adiabatic. Between approximately 30 and $50 \mathrm{~km}$, the decrease of temperature along altitude is slower than expected by the adiabatic gradient (up to $4 \mathrm{~K} / \mathrm{km}$ ), resulting in the formation of a region very stable against convective overturning (Seiff et al. 1985). 
The entire planet is covered by layers of aerosols at heights between $49-70 \mathrm{~km}$. The occurrence of clouds allows us to track a number of dynamical phenomena. A main feature of atmospheric general circulation is the global wind super-rotation at intermediate latitudes, with velocities between $60-120 \mathrm{~m} / \mathrm{s}$ (Hueso et al. 2015). Upwelling of absorbing materials from deeper cloud regions associated with a Kelvin-like trapped wave was proposed to justify the $90^{\circ}$-tilted " $Y$ pattern" observed in UV images extending symmetrically on the two hemispheres up to $45^{\circ}$ (Peralta et al. 2015). Despite thick clouds, a small but non-negligible part of the solar flux penetrates to the surface and heats the atmosphere due to the greenhouse effect. The thermal structure of Venus atmosphere above the $55 \mathrm{~km}$ level was extensively studied by VIRTIS (Visible and Infrared Thermal Imaging Spectrometer) onboard Venus Express (see Limaye et al. 2017, 2018, and references therein), with measurements on the night-side, preferentially over the southern polar regions. VIRTIS data confirmed and provided further details about the mesospheric thermal fields already known from the data of the Venera 15 Fourier Spectrometer (Zasova et al. 1999).

At night, the temperature field exhibits a high degree of symmetry between the two hemispheres, as expected from the very small axial tilt. At levels around $55 \mathrm{~km}$, air temperature tends to decrease monotonically from the equator to the poles (from 290 to $240 \mathrm{~K}$ ), as expected by intense absorption of UV radiation by clouds at the subsolar points. At the $65 \mathrm{~km}$ level, dynamical effects begin to dominate, with two symmetric "cold collars" showing a minimum temperature of $220 \mathrm{~K}$ at $65 \mathrm{~S}$ and $65 \mathrm{~N}$. Above $70 \mathrm{~km}$ and at least up to $90 \mathrm{~km}$, equatorial regions are colder than polar regions, with monotonically increase at fixed altitude. This behaviour is consistent with a global scale Hadley circulation, where polar heating is caused by adiabatic compression of descending air. Mapping by VIRTIS unambiguously reveals patterns in local-time/latitude air temperature averages on the night side of the planet, tentatively associated with wave activities. Day-to-day variability was also observed, being particularly evident in the cold-collar region. On the dayside of Venus a few hundreds of high vertical resolution temperature profiles were obtained from radio-occultation measurements of the VeRA experiment (Tellmann et al. 2009). The overall thermal structure seems consistent to the night hemisphere, but the resulting coverage remains sparse.

Air temperatures at higher altitudes (between 90 and $110 \mathrm{~km}$ ) at all local times are still poorly constrained by available data, but suggest very strong variations. This variability is possibly related to the overall change in global circulation pattern (from Hadley to solar/antisolar) occurring at this altitude. Above $120 \mathrm{~km}$, the dayside atmosphere is dominated by absorption of UV radiation by molecules, with locations of peaks values of temperature strictly following the subsolar point. The solar/antisolar temperature gradient around $120 \mathrm{~km}$ was found to be around $35 \mathrm{~K}$.

Aerosols and Clouds The most striking feature of the Venusian atmosphere is the thick coverage of clouds that permanently preclude the visual observation of the surface, with a total opacity at visible and near-IR wavelengths that varies from 20 to 40 (for a review see Titov et al. 2018). Data from Pioneer probe nephelometer is the primary standard for inferring Venus' cloud characteristics and still the best available today (Knollenberg and Hunten 1980). Aerosol populations encountered by the nephelometer in its descent from the space towards the surface corresponded first to a population of sub-micron haze (so-called "mode 1" particles), with particle detection beginning at an altitude of $\sim 100 \mathrm{~km}$ and lower. A second, larger component (modal radius of $\sim 1.2 \mu \mathrm{m}$ ) is evident below $70 \mathrm{~km}$. This component ("mode 2") is the main constituent (in terms of mass) of the upper clouds of Venus, that is typically observed from space in visible and infrared. A local minimum in cloud opacity at about $57 \mathrm{~km}$ marks the transition at the middle/lower cloud deck, where larger particles 
$\left(r_{m}=3-5 \mu \mathrm{m}\right.$, "mode 3") are found. At $48 \mathrm{~km}$ of altitude a sharp decrease in aerosol opacity is observed, and below only much optically thinner diffuse haze and possibly discrete clouds of uncertain nature can be found (Grieger et al. 2003). In situ analysis (Hoffman et al. 1980), as well as remote IR (Zasova et al. 2007) and polarimetric measurements (Hansen and Hovenier 1974) identified a liquid mixture of sulfuric acid and water as the main constituent of haze and upper clouds: consistently, the clearing observed at the altitude of $48 \mathrm{~km}$ occurs where the temperature in the Venus environment allows the sulphuric acid to evaporate. Other constituents must be present as well: UV observations show high contrasting details, demonstrating the existence of a still unidentified UV absorber, strongly variable in space and time (Markiewicz et al. 2007); chlorine, phosphorus, and iron in the deeper clouds form still unidentified components as inferred by the measurements by instruments onboard the VEGA balloon (Andreichikov 1987).

The large suite of Venus Express instruments operating from thermal IR $(5 \mu \mathrm{m})$ to UV enabled a series of studies, mostly focused on upper clouds and hazes. Among the notable results, the decrease of upper cloud heights and scale heights poleward from about $50^{\circ}$ in longitude (Ignatiev et al. 2009; Haus et al. 2014), the detection of detached haze layers and larger particles ( $1 \mu \mathrm{m}$ above) above the cloud top at $75 \mathrm{~km}$ (Wilquet et al. 2012; Luginin et al. 2016), variations in size and refractive indices with local time and latitude (Shalygina et al. 2015; Wilson et al. 2008). More spare are the studies about deeper aerosols: Magurno et al. (2017) found an increase of "mode 3" particle size with latitude, that is qualitatively consistent with the increase of total opacity of deep clouds towards the pole reported by Cardesín Moinelo et al. (2008) from VIRTIS data.

The BepiColombo Flybys Near and during the time of flybys, Venus's day and night side atmospheres will be observed by MERTIS (radiometer and spectrometer) and PHEBUS spectrometer that will make complementary measurements, and will work together with the Akatsuki spacecraft presently orbiting around Venus, and with the Earth-based telescopes (see Sect. 5.2). MERTIS (Hiesinger et al. 2020, this journal) will allow sensing the atmosphere from the top to an altitude of about $60 \mathrm{~km}$ above the surface, just within the upper cloud deck. It will also be able to detect mid-infrared thermal emissions from the cloud top level at daytime, and from below the cloud top at nighttime. In particular, the infrared spectrometer TIR in the range 7-40 $\mu \mathrm{m}$ allows sounding the middle atmosphere of Venus in the altitude range 55-100 km and its cloud layer (specifically $\mathrm{CO}_{2}, \mathrm{SO}_{2}$, and $\mathrm{H}_{2} \mathrm{SO}_{4}$ have absorption bands in the 7-14 $\mu \mathrm{m}$ range covered by the spectral channel TIS of MERTIS). Up to now, only a very limited set of observations of Venus in the thermal infrared spectral range is available.

PHEBUS spectrometer (Quémerais et al. 2020), designed to observe the weak emissions of the Hermean exosphere, is not optimized to observe the bright dayside atmosphere of Venus with the FUV channel (145-315 nm), that may damage the detector. In addition, due to the power limit, only one detector (EUV at 55-155 nm, or FUV) can be switched on at a time during the flybys (but they can be used both, alternatively). However, both NUV channels (at 404 and $422 \mathrm{~nm}$ ) can be switched on at the same time. PHEBUS plans to perform two different observation modes during the Venus flyby closest approach periods: (a) star occultation at CA in FUV range at nightside (in both flybys), and (b) EUV airglow observations at high altitudes (during the second flyby). Stellar occultation is done in inertial mode and could be performed at EUV or at FUV if pointing at the nightside, to avoid scatter light from the Venusian atmosphere (Montmessin et al. 2011). It will allow deriving $\mathrm{CO}_{2}$ maps; while $\mathrm{O}_{3}$ and $\mathrm{SO}_{2}$ could be derived from the FUV channel.

MGNS neutron and gamma spectrometers (Mitrofanov et al., this journal) will also contribute to atmospheric investigations by detecting elemental composition in terms of $\mathrm{C}, \mathrm{O}$, 
$\mathrm{N}$, and $\mathrm{H}_{2} \mathrm{O}$, indicative for the complex processes of diffusion, vertical mixing and chemical processing occurring in the Venus atmosphere.

First Venus Flyby The spacecraft approached the planet from the solar direction, over the dayside. The closest approach occurred above the evening terminator of the planet (Fig. 15), and then the spacecraft moved away from the planet to the anti-solar direction, over the night side. During the approaching phase, MERTIS started to resolve Venus from a distance of 1.4 million km when Venus size was about the same as seem from Akatsuki spacecraft, and went on until 4 hours before CA. At the closest distance, MERTIS gained a spatial resolution of about $6 \mathrm{~km}$ at the cloud top, i.e. higher than all the currently existing datasets. The contemporary radiometric data obtained provided insights into temperature profiles both on the illuminated and the non-illuminated hemisphere. Between 7 and 4 hours before CA, MERTIS performed close-up dayside observations from late morning to late afternoon via noontime on Venus at low latitudes of the southern hemisphere. Due to the $100 \%$ cloud cover on the planet, most of thermal emissions are corresponding to temperatures at the upper cloud level atmosphere (60-70 km) (Zasova et al. 2007). The shorter wavelength edge of the $15-\mu \mathrm{m} \mathrm{CO} 2$ band covered by TIS/MERTIS will help to retrieve temperature profiles from the cloud tops to slightly above using the inversion methods of radiative transfer (Rodgers 2000). Simultaneous cloud top structures and $\mathrm{SO}_{2}$ gas abundances above the clouds can be estimated from the observed spectra over the 7-14 $\mu \mathrm{m}$ range of TIS/MERTIS. This spectral range observation from space will be the first measurement of this kind after Venera missions in 1980s (Zasova et al. 2007).

PHEBUS planned to perform star occultation in the night side of Venus, shortly after CA time, by observing perpendicular to the dawn and dusk limbs but unfortunately no night side observations were obtained. Stellar occultations have been performed in the past in a systematic way by SPICAV-UV onboard Venus Express at the nightside. Vertical profile of aerosols of the haze layer, $\mathrm{CO}_{2}$ density and temperature have been derived from stellar occultations between 80-140 km (Bertaux et al. 2007; Piccialli et al. 2015). An ozone layer has also been detected between $90-105 \mathrm{~km}$ from detailed inspection of these stellar observations at the strongest Hartley band around $250 \mathrm{~nm}$ (Montmessin et al. 2011). However, such an identification has been obtained only sporadically. Night side distribution of sulfur dioxide $\left(\mathrm{SO}_{2}\right)$ content at the same altitude range was retrieved from SPICAV-UV star occultations as well (Belyaev et al. 2017). Systematic SPICAV measurements in $\mathrm{SO}_{2}$ absorption bands at 190-220 nm and 270-290 nm provided a study of the annual variability for 2006-2014. The star occultation by PHEBUS, possessing a twice better spectral resolution, should have complemented such a long-term trend. Synergic observations of the Venusian atmosphere from MERTIS on the dayside, and from PHEBUS on the nightside, may help also in the study of the atmosphere from the chemical and dynamical point of view, for they should be able to observe how the chemistry changes before terminator, soon after, and shortly before entering again into light. Additionally, PHEBUS performed dayside disk integrated albedo in FUV when faraway from Venus (in late August-early September 2020) with the goal to monitor albedo variability over time; the visible channel was used too to measure global average $\mathrm{SO}_{2}$ content.

Second Venus Flyby For the second flyby at Venus, the spacecraft will approach the planet from its night side, and CA will occur again near the evening terminator, but slightly shifted towards the late afternoon side with respect to the first flyby (Fig. 15). Then the spacecraft will move away from the planet towards the morning direction. The minimum altitude will be now only about $550 \mathrm{~km}$. The close-up observations by MERTIS will be 
done from early night side to evening terminator at low latitudes, with a similar strategy as 1 st flyby. At the CA, spatial resolution at the cloud top will be about $360 \mathrm{~m}$ providing an unprecedented detailed look at the distribution of minor species and the cloud structure in the atmosphere of Venus. PHEBUS will observe the exosphere of Venus with the EUV channel. The Venusian exosphere is mainly composed of atomic $\mathrm{H}, \mathrm{He}$ and $\mathrm{O}$. Exospheric $\mathrm{H}$ and $\mathrm{O}$ are composed of a population at thermal equilibrium with the atmosphere and a suprathermal population. While the hot $\mathrm{H}$ population is observed systematically by UV spectrometers (Chaufray et al. 2012 and references therein), the hot O population has only been observed by Pioneer Venus Orbiter UV Spectrometer (Nagy et al. 1981) and by Venera 11 (Bertaux et al. 1981). Several emission lines could be observed, depending on the altitude of the line of sight of PHEBUS. The hydrogen Lyman- $\alpha$ brightness, should vary between $\sim 2$ to $\sim 30 \mathrm{kR}$ for a line of sight tangent altitude from 500 to $2000 \mathrm{~km}$ based on SPICAV/Venus Express observations (Chaufray et al. 2012; Chaufray and Bertaux 2015) and then it could be detected by PHEBUS at all altitude during the flyby; the Lyman- $\beta(\sim 300$ times weaker) could be detected from $1000 \mathrm{~km}$ altitude. Extrapolated from disk observations by Cassini/UVIS during the Venus flyby (Gérard et al. 2011a), the He brightness should be of only a few Rayleighs at $1000 \mathrm{~km}$, due to its fast vertical decrease (scale height $\sim 70 \mathrm{~km}$ ) and then difficult to detect; nonetheless, it will surely be worth to try. The $\mathrm{O}$ emission at 130.4 nm, based on Pioneer Venus Orbiter (PVO) observations (Nagy et al. 1981) should be of few hundreds Rayleighs at $1000 \mathrm{~km}$. It could be a new first opportunity, since PVO, to detect the hot oxygen on Venus, not detected with SPICAV/Venus Express. Unfortunately, the geometry of this second flyby should prevent PHEBUS to detect other lines (like CO, and C) in the EUV range (Gérard et al. 2011b), but EUV observations faraway from Venus could be done to observe them. Periodicities in the EUV dayglow (O II $83.4 \mathrm{~nm}$, O I 130.4 and $135.6 \mathrm{~nm}$ ) in the Venusian upper atmosphere, possibly associated to periodicities in oxygen or photoelectrons fluxes in the thermosphere due to planetary waves or gravity waves, have been detected by EXCEED aboard Hisaki (Masunaga et al. 2015) and will be investigated using consecutive observations by PHEBUS. Stellar occultations could be performed before or after the closest approach as done during the first flyby.

\subsubsection{Induced Magnetosphere and Plasma Environment}

Unlike the Earth and Mercury, Venus has no intrinsic dipole magnetic field and thus is a perfect prototype of the solar wind interactions with unmagnetized planets (e.g. Futaana et al. 2017). Without the shielding of an intrinsic dipole magnetic field, the impinging solar wind plasma can easily approach the planet and interact directly with the upper atmosphere. Figure 19 illustrates the major features of the solar wind interaction with Venus' upper atmosphere as deduced from the solar cycle maximum measurements obtained by Pioneer Venus Orbiter (PVO). The interaction leads to various regions and boundaries, such as the bow shock, magnetosheath region, induced magnetosphere and ionosphere on the upstream side, and a complex magnetotail in the downstream region.

Ionosphere On the dayside of Venus, the upper atmosphere is ionized by solar extreme ultraviolet radiation and solar wind particle impacts, leading to the formation of an ionosphere (Schunk and Nagy 2009), observed for the first time in 1967 by Mariner 5 (Kliore et al. 1967; Fjeldbo and Eshleman 1969). $\mathrm{CO}_{2}^{+}$is the primary ion created by photoionization, and it quickly transforms into $\mathrm{O}_{2}^{+}$and $\mathrm{O}^{+}$due to charge exchange reactions. As a result, $\mathrm{O}_{2}^{+}$is the major ion species at low altitudes while $\mathrm{O}^{+}$becomes most abundant at high altitudes. The peak density of $\mathrm{O}_{2}^{+}$at low solar zenith angles is observed at $130-150 \mathrm{~km}$ 


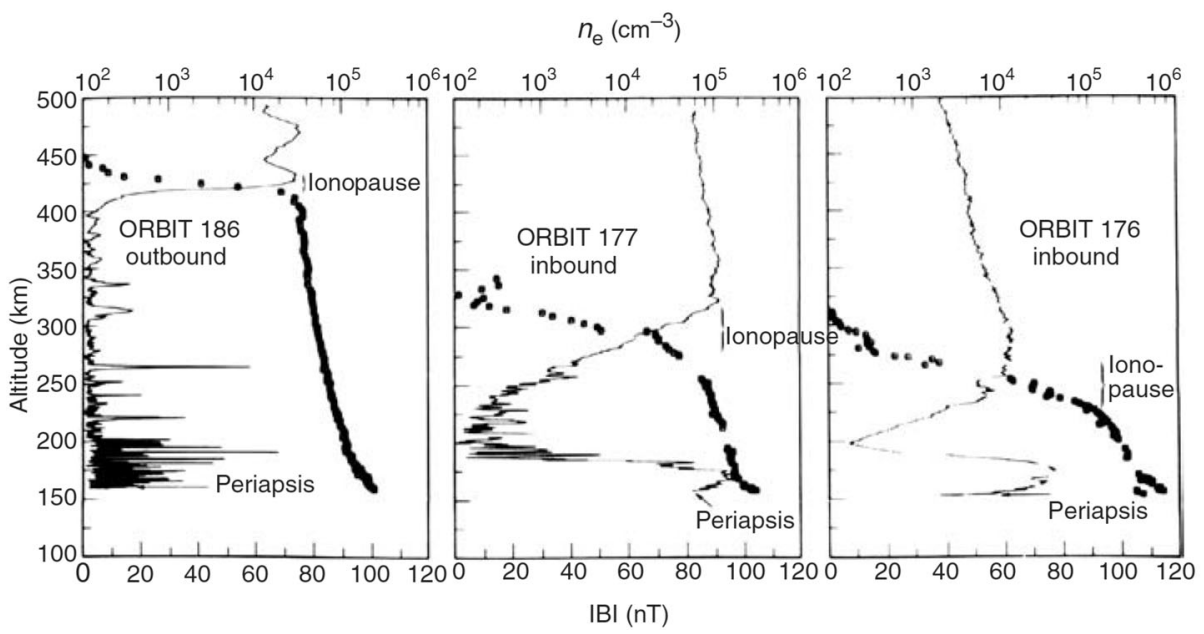

Fig. 18 PVO measured altitude variations of magnetic field strength (thin line) and electron density (circles) in the Venusian ionosphere for low to moderate solar wind dynamical pressure on orbit 186 (left), moderate to high solar wind dynamical pressure on orbit 177 (middle), and very high solar wind dynamical pressure on orbit 176 (right) (from Elphic et al. 1981)

altitude, and above $200 \mathrm{~km}$ for $\mathrm{O}^{+}$. Mariner 5 observations showed, surprisingly, a highly variable but significant ionosphere on the nightside of Venus, which was later quantified by observations from the PVO in 1978-1992 (Miller et al. 1980). More interestingly, PVO also observed ionospheric density holes on the Venusian nightside, possibly caused by the pressure balance between observed radial magnetic fields and ionospheric thermal pressure (Brace et al. 1982). Though Venus has no significant intrinsic magnetic field, at times of high solar wind dynamic pressure a significant $(\sim 100 \mathrm{nT})$ horizontal magnetic field is induced in the ionosphere (examples of both situations are shown in Fig. 18, Elphic et al. 1981). The interplanetary magnetic field (IMF) penetrates into the ionosphere, especially during solar cycle minimum, and it can significantly modify the ionospheric density profile. When the thermal pressure of the ionosphere is higher than the solar wind pressure (especially during the solar cycle maximum), the magnetic field drops sharply to nearly zero across the ionopause (Fig. 18, left panel). As a consequence, also the position of the ionopause may vary significantly from about 250 to $450 \mathrm{~km}$ altitude: the Venus Express data showed that the ionosphere is highly magnetized (about $95 \%$ of the time), but only $15 \%$ during solar cycle maximum (Zhang et al. 2008).

The Flow of Ions Across the Terminator Since ions are expected to recombine with the ambient electrons during the night of Venus (lasting 58 Earth days), a steady supply of ions from the dayside must exist to maintain the nightside ionosphere. It was already suggested by McElroy and Strobel (1969) that the transterminator ion flow can deliver this supply. Such a significant quantity of ions is indeed transported from the dayside to the nightside across the terminator of Venus and was first observed by the Orbiter Retarding Potential Analyzer (ORPA) onboard PVO using only a few orbits of observations (Knudsen et al. 1980). These first observations showed a chaotic but generally tailward motion of $\mathrm{O}^{+}$ions with velocities of $1-8 \mathrm{~km} / \mathrm{s}$. Above $200 \mathrm{~km}$ altitude $\mathrm{O}^{+}$is the dominant ion species at the terminator, while $\mathrm{O}^{+}$and $\mathrm{H}^{+}$ions contribute less than $10 \%$ to the total ion density (Miller et al. 1980). The analysis of these data from 3.5 years of PVO observations showed that the 


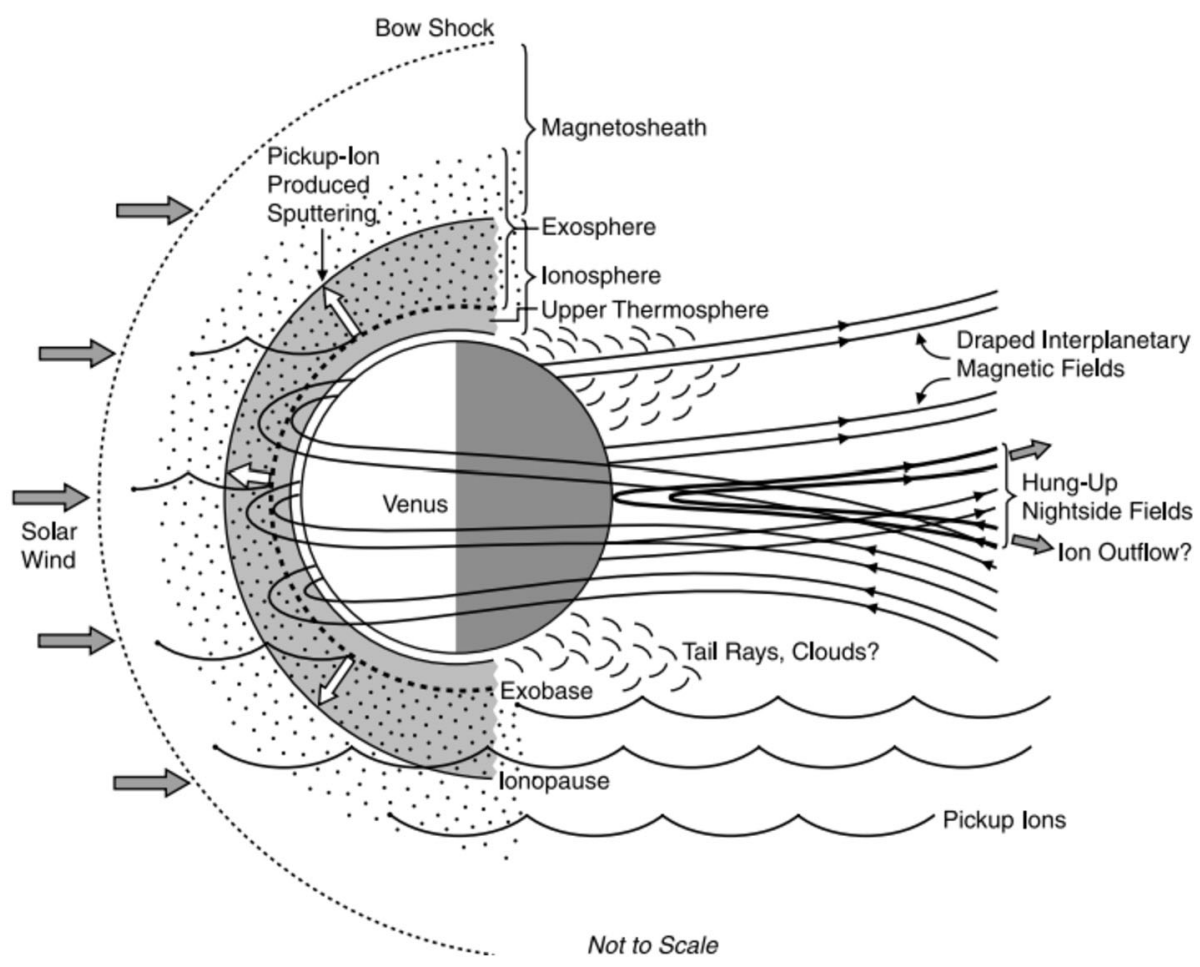

Fig. 19 Schematic diagram of the solar wind interaction with Venus induced magnetosphere and upper atmosphere (from Russell et al. 2007)

transterminator ion flow at Venus has a mean velocity of about $1-2 \mathrm{~km} / \mathrm{s}$ at $300 \mathrm{~km}$ altitude and increases to $4 \mathrm{~km} / \mathrm{s}$ at $600 \mathrm{~km}$ altitude (Knudsen et al. 1982; Knudsen and Miller 1992). Transient velocities up to $8 \mathrm{~km} / \mathrm{s}$ have been observed too. The mean transterminator ion flux observed $\left(2 \cdot 10^{9} \mathrm{~cm}^{-2} \mathrm{~s}^{-1}\right)$ is sufficient to maintain the nightside ionosphere of Venus. The observations of the PVO ORPA experiment summarized in Miller and Whitten (1991) showed that the velocity of ions on the dayside increases with solar zenith angle and that there is a dawn-dusk asymmetry in the flow-pattern for which at the time no explanation was found. More recently, Fox and Kasprzak (2007) reported a dawn-dusk asymmetry in the thermosphere that may be related to the asymmetry in the ionospheric flow. Also, Fox (2008) showed that new models of the dayside ion production at Venus are in agreement with the transterminator flux at Venus but cannot explain the loss rates of more than $10^{25}$ ions $/ \mathrm{s}$ as observed in the tail of Venus by the Venus Express ASPERA-4 instrument (Barabash et al. 2007a). It was suggested by Cravens et al. (1982) and Whitten et al. (1982) and shown by Miller and Knudsen (1987) that the transterminator flux at Venus is controlled by the terminator ionopause altitude and thus by the solar wind pressure. Comprehensive reviews of the interaction of the Venus atmosphere with the solar wind can be found in Dubinin et al. (2011) and Futaana et al. (2017).

The Ionotail As mentioned above, observations by the PVO ORPA instrument (Miller et al. 1980) showed that above $200 \mathrm{~km}$ of altitude $\mathrm{O}^{+}$is the dominant ion species at the terminator, while $\mathrm{O}^{+}$and $\mathrm{H}^{+}$ions contribute less than $10 \%$ to the total ion density. Different 

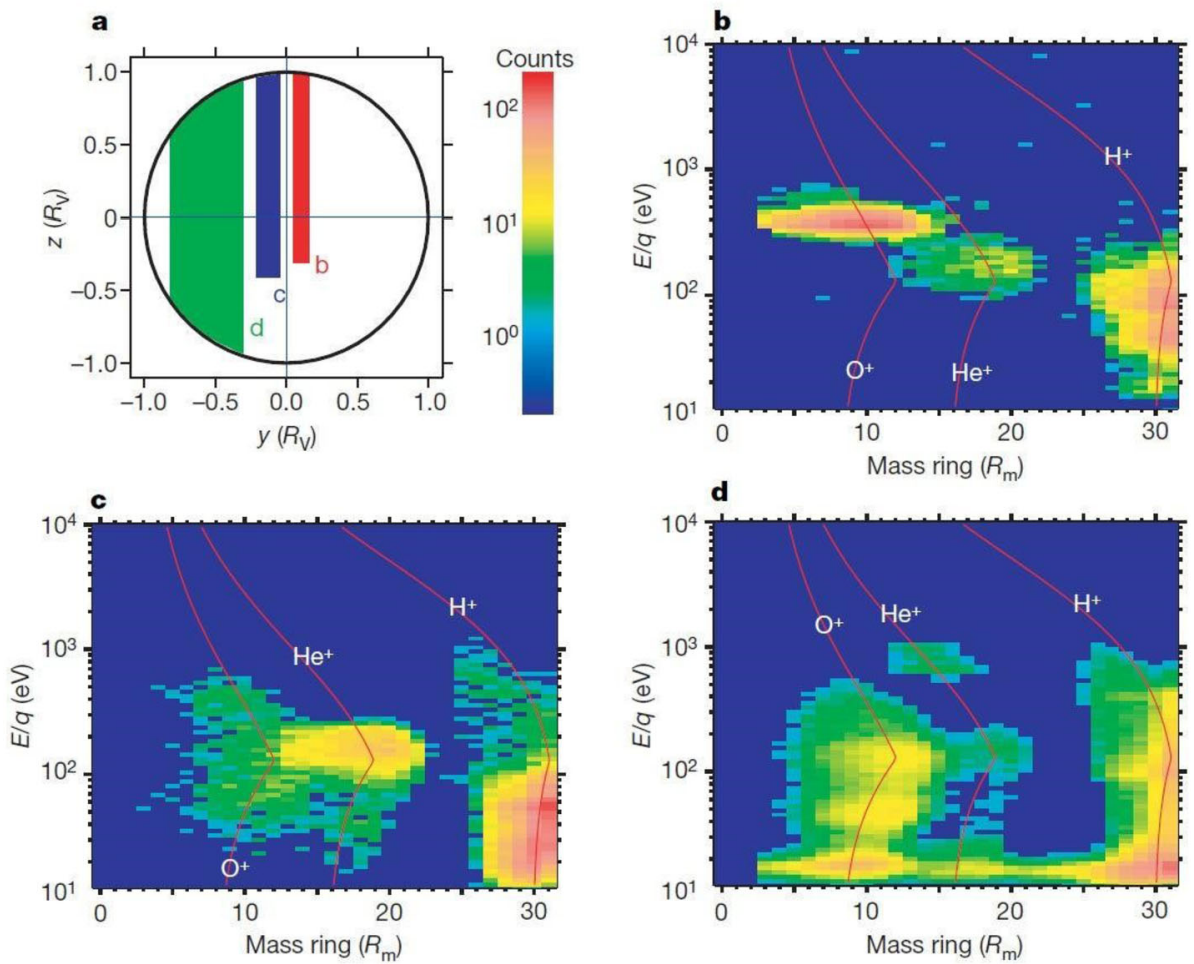

Fig. 20 Composition and energy distribution of the escaping plasma as observed by ASPERA-4 on VEX over 33 orbits during the period from 18 May to 30 December 2006: (a) position of the spatial regions (b), (c) and (d); (b) plasma sheet matrix for the $+Y$ lobe; (c) a plasma sheet matrix for the $-Y$ lobe and (d) a matrix for the IMB layer. Vertical axes are energy/charge $(E / q)$, the horizontal axes are the position of the ion impact on the detector (a sensor mass ring, $R_{m}$ ), and the color codes the counts that were accumulated over all directions and averaged over occurrences. The red lines represent constant mass lines (from Barabash et al. 2007a)

estimates based on observations by PVO at distances up to $10 R_{V}$ put the ion loss rate from Venus at about $10^{26} \mathrm{H}^{+}$ions/s and $1.4 \cdot 10^{25} \mathrm{O}^{+}$ions/s (Knudsen and Miller 1992). But ionospheric models (Fox 2008) suggest that depending on the strength of solar EUV also $\mathrm{C}^{+}, \mathrm{N}^{+}, \mathrm{He}^{+}, \mathrm{CO}^{+}, \mathrm{CO}_{2}^{+}$may be observed at altitudes above $400 \mathrm{~km}$. In a rare crossing of the Venus ionotail at the L1 point ( $750 R_{V}$ downstream) by the SOHO spacecraft in June 1996, Grünwaldt et al. (1997) identified $\mathrm{C}^{+}, \mathrm{N}^{+}$, and $\mathrm{He}^{+}$, while the SOHO/CELIAS instrument was not sensitive enough to detect heavier ions.

The MIMI CHEMS instrument onboard Cassini spacecraft, when passed Venus at a minimum altitude of $603 \mathrm{~km}$ on 24 June 1999, observed pick-up of $\mathrm{O}^{+}$and $\mathrm{C}^{+}$ions at pericenter (Krimigis et al. 1999) but no quantitative observations have been published. The energetic particle spectrometer EPS onboard MESSENGER spacecraft, during its flyby at Venus on 5 June 2007, did not discover significant fluxes. The Fast Imaging Plasma Spectrometer (FIPS) discovered pick-up ions of possible Venusian origin, but unfortunately no further analysis of these observations has been published. Hence, the most recent observations of the loss of ions from Venus come from the ASPERA-4 instrument (Barabash et al. 2007b) on Venus Express (VEX, 2006-2014). Barabash et al. (2007a) report observations of $\mathrm{O}^{+}$, $\mathrm{H}^{+}$and $\mathrm{He}^{+}$at flux ratios of $\mathrm{Q}\left(\mathrm{He}^{+}\right) / \mathrm{Q}\left(\mathrm{O}^{+}\right)=0.07$ and $\mathrm{Q}\left(\mathrm{H}^{+}\right) / \mathrm{Q}\left(\mathrm{O}^{+}\right)=1.9$ (see Fig. 20 

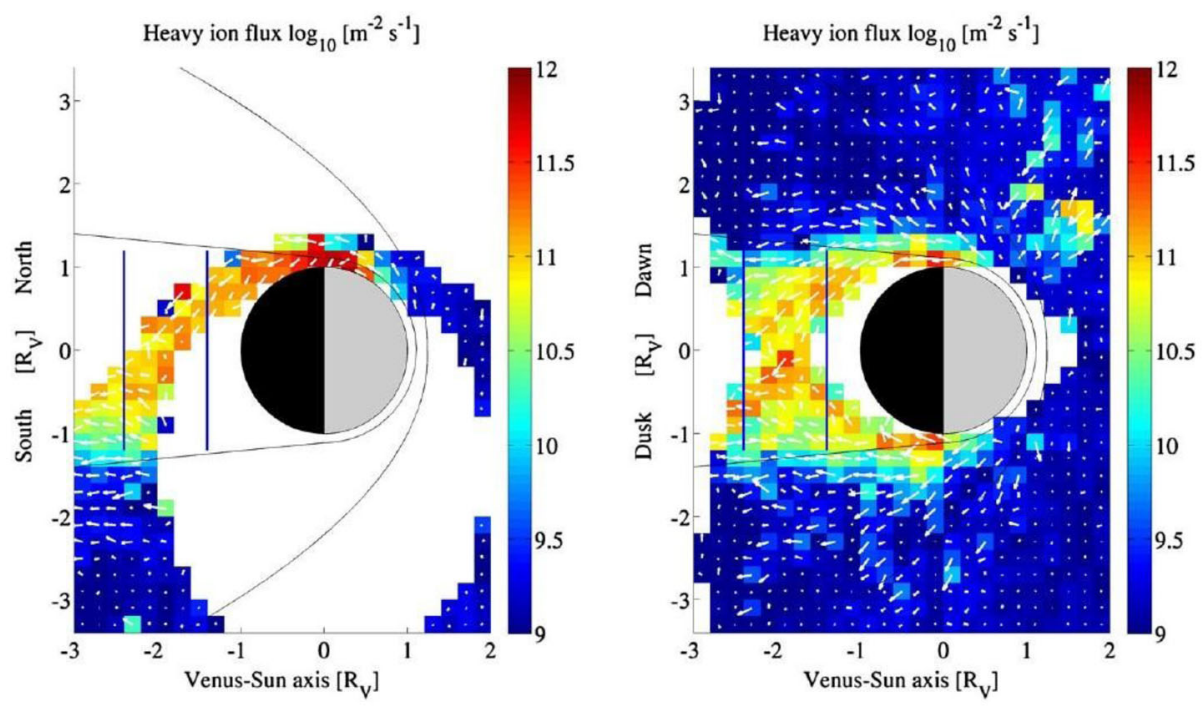

Fig. 21 Heavy ion flux intensity and direction around Venus in the VSO frame as observed by ASPERA-4 on VEX (years 2006-2012) in $X Z$ plane (left) and $X Y$ plane (right). Color scale is the logarithm of the total average omnidirectional flux in each bin in $\left[\mathrm{m}^{-2} \mathrm{~s}^{-1}\right]$. White arrows indicate the average direction of the flow, and the arrow length corresponds to net flux (from NordströM et al. 2013)

left side). The $\mathrm{Q}\left(\mathrm{H}^{+}\right) / \mathrm{Q}\left(\mathrm{O}^{+}\right)$was recently re-assessed by Persson et al. (2018), where they found a significant solar cycle dependence: in the solar minimum the ratio is 2.6, while the solar maximum it becomes 1.1. Nevertheless, the ratio of $\mathrm{Q}\left(\mathrm{H}^{+}\right) / \mathrm{Q}\left(\mathrm{O}^{+}\right)$of 2 on average is in agreement with the predictions of Lammer et al. (2006) that with any lost oxygen atom, two hydrogen atoms must be lost because they derive from water molecules. The total loss rates were later better quantified by Fedorov et al. (2011) to be $\mathrm{Q}\left(\mathrm{H}^{+}\right)=7.1 \cdot 10^{24} / \mathrm{s}$, $\mathrm{Q}\left(\mathrm{O}^{+}\right)=2.7 \cdot 10^{24} / \mathrm{s}$ and $\mathrm{Q}\left(\mathrm{He}^{+}\right)=7.9 \cdot 10^{22} / \mathrm{s}$ and updated by NordströM et al. (2013) to the values to $\mathrm{Q}\left(\mathrm{H}^{+}\right)=1.4 \cdot 10^{25} / \mathrm{s}$ and $\mathrm{Q}\left(\mathrm{O}^{+}\right)=5.2 \cdot 10^{24} / \mathrm{s}$. Dubinin et al. (2013) derived the density and velocity of the $\mathrm{O}^{+}$in Venus's tail, as shown in Fig. 21 (note that the density of the $\mathrm{O}^{+}$remains well below $1 \mathrm{~cm}^{-3}$ ). More details of the Venus Express observations are reviewed in Futaana et al. (2017). Unfortunately the ASPERA-4 IMA instrument was not designed to separate minor species like $\mathrm{C}^{+}$or $\mathrm{N}^{+}$from $\mathrm{O}^{+}$. Also the separation between $\mathrm{O}^{+}$, $\mathrm{O}_{2}^{+}, \mathrm{CO}_{2}^{+}$and heavier species is ambiguous, and therefore loss of ions heavier than $\mathrm{O}^{+}$has not been reported so far. Recently ASPERA-4 measurements in the Venusian tail revealed an unexpected reverse flow, namely, a bulk plasma flow towards Venus. The amount of the reverse flow is significant, which influences the net outflow flux from the Venusian ionosphere to space (Kollmann et al. 2016; Persson et al. 2018). The reverse flow is frequently observed in the solar maximum period, and because of that the net outflow flux is reduced in the solar maximum to $2.0 \cdot 10^{24} \mathrm{~cm}^{-2} \mathrm{~s}^{-1}$ (compared to $2.9 \cdot 10^{24} \mathrm{~cm}^{-2} \mathrm{~s}^{-1}$ in the solar minimum). Interestingly, there are frequently cases where the proton and the oxygen have different bulk flow directions. The mechanism that produces the reverse flow is not yet identified, while several mechanisms can be considered, for example, the magnetic reconnection (Zhang et al. 2012) or manifestation of single particle motions.

High-Energy Particle Shadowing by an Induced Magnetosphere One question that can potentially be studied with BepiColombo at Venus is magnetic shadowing of high- 
energy particles caused by the induced magnetosphere. This effect was studied at Mars by McKenna-Lawlor et al. (2012), who found shadowing to be effective up to MeV energies by comparing global hybrid simulations to data recorded by the SLED instrument aboard Phobos-2 (McKenna-Lawlor et al. 1990). Different parts of the induced magnetosphere are expected to contribute differently to particle shadowing. For energetic particles with speeds much larger than the bulk speeds of the flow, the most important agents providing shadowing are the enhanced magnetic field and the planet itself. We, therefore, expect the greatest effects from the magnetosheath, the magnetic pileup boundary and the planet's shadow, depending on the energy, particle species and conditions in the solar wind. Besides the energy range and the orbit, also the directional resolution and coverage of the instrument observing the flux will be important. BepiColombo/SIXS observes the electron (60-3000 keV) and proton (1-30 MeV) flux in five orthogonal directions, of which three remain unobstructed in cruise configuration. With a single spacecraft flying by the planet, the study has to be backed up by a global simulation of the planetary plasma environment to provide context and a prediction to be verified by observations. In addition to an instrument capable of measuring magnetic shadowing effects, high fluxes of energetic particles will be necessary for successful studies of magnetic shadowing. Solar activity during the flybys is not expected to be at maximum, but the second flyby will most likely occur already well into the rising phase of Solar Cycle 25, increasing the chances of observing high fluxes of solar energetic particles during the flyby.

Solar Wind Charge Exchange Induced X-Rays Interpretation of in situ space plasma measurements is complicated by mixed spatial and temporal variations and orbital coverage. Potential remote sensing techniques of planetary plasma environments addressing these issues include the Solar Wind Charge eXchange (SWCX) induced X-rays. The planetary SWCX X-rays are generated when multiple charged solar wind heavy ions are highly excited in charge transfer reactions with exospheric neutral species and transition to lower energy states emitting soft X-rays occurs (Dennerl 2010). The main source of X-rays at Venus is the fluorescent scattering of the solar X-rays in the atmosphere, but the SWCX induced X-rays can be distinguished from the total flux during favorable low solar X-ray conditions typical near solar minimum. The Venus SWCX X-rays are very confined to the limb making it challenging to distinguish them from the fluorescent scattering, compared to the more extended exosphere of Mars and around comets (Gunell et al. 2007). The X-Ray Spectrometer (XRS) observed a decrease in the X-ray count rate as Venus blocked the background sky during MESSENGER's Venus flyby on 5 June 2007, but no emission related to the planetary environment was seen, which was explained by the instrument's low-energy cutoff at $\sim 1 \mathrm{keV}$ (McNutt et al. 2008). SIXS-X may experience the same challenge due to its spectral range of $1-20 \mathrm{keV}$, but the detection probability is also influenced by the solar $\mathrm{X}$-ray irradiance as well as the flyby geometry. Other ion sensors, as SERENA MIPA and PICAM, and plasma sensors from Mio may significantly contribute.

First Venus Flyby During the first Venus flyby, BepiColombo was embedded in the solar wind magnetoplasma upstream of the bow shock. Therefore, various aspects of solar wind turbulence can be studied depending on the available data. They include the scaling properties of the magnetic energy power spectra and their comparison to known features at $1 \mathrm{AU}$ (see e.g. Bruno et al. 2009; Narita et al. 2011; Sahraoui et al. 2013), the nature of high frequency turbulence and possible wave-particle interactions processes (Sahraoui et al. 2009, 2010), and the nature of the waves (e.g., cyclotron wave) in the upstream pickup ions (or foreshock) region (Delva et al. 2015). From Venus Express data it is expected that when the 
spacecraft moves towards the bow shock the magnetic field strength will increase as well as the wave activity, as shown by Guicking et al. (2010). Downstream of the bow shock, two different situations can be met depending on the orientation of the interplanetary magnetic field with respect to the shock normal: a quasi-perpendicular or a quasi-parallel bow shock. In the case of the quasi-perpendicular bow shock it is expected that downstream ion temperature anisotropy will develop, which can trigger various ion-scale instabilities such as mirror modes (Volwerk et al. 2008a,b, 2016; Bader et al. 2019). These waves have been observed beyond Venus's terminator and they can either be generated locally or are transported by the plasma flow in the magnetosheath (Sahraoui et al. 2003). In case of a quasi-parallel bow shock various ultra-low frequency waves can be generated in the foreshock region and propagate or be transported downstream (Fränz et al. 2017). The spacecraft crossed the bow shock around the time of closest approach and will enter the ionotail about $40 \mathrm{~min}$ later at a distance of $5 R_{V}$. This offers the opportunity to study the magnetotail dynamics. The magnetotail of Venus is prone to similar dynamics as the Earth's magnetotail, where magnetic reconnection plays an important role in magnetic flux and plasma particle transport (see e.g. Volwerk et al. 2009, 2010; Zhang et al. 2012; Huang et al. 2018). Therefore, it can be expected to see plasmoids travelling tailward during this flyby as also shown by the MHD model with a heavy ion flux of about $10^{8}\left[\mathrm{amu} /\left(\mathrm{cm}^{2} \mathrm{~s}\right)\right]$. These relatively high heavy ion fluxes should have allowed PICAM to measure the magnetospheric ions during the first flyby. Also, the MSA ion spectrometer on Mio may measure low count rates of heavy ions from its restricted field of view within the MOSIF shield. This may have allowed for the first time to measure the escape of minor ions like $\mathrm{C}^{+}$or $\mathrm{N}^{+}$. The magnetic reconnection studies can be completed by identifying the nature of the high frequency waves (e.g., whistlers) that are responsible for the processes of wave-particle interaction near the reconnection site (ion and electron diffusion regions), as generally done in similar studies in the Earth's magnetosphere (Huang et al. 2016). Such identification can be achieved by measuring high frequency magnetic fluctuations, which is possible thanks to the Search-Coil (SC) magnetometers (Low-Frequency Search Coils LF-SC and Dual-Band Search Coil DB-SC) of the Mio/PWI (Plasma Wave Investigation) instrument (Kasaba et al. 2020). In particular, the DB-SC instrument can measure the Z-component of the magnetic field fluctuations up to $640 \mathrm{kHz}$, which should allow covering all the local characteristic frequencies of the plasma (e.g., plasma frequency, electron cyclotron frequency). This type of studies can actually be achieved across all the regions (shock, magnetosheath and ionotail) explored by BepiColombo. So far, no such high frequency wave measurements exist in the Venusian environment. MPO-MAG data are fundamental to such investigations.

The outcome of such studies will be further strengthened by any additional plasma data (ions and electrons) that would come from the Mio/MPPE instrument. In addition to observations of magnetic reconnection and heavy ion fluxes during the first Venus flyby, it is also interesting to further study the Venusian magnetotail current sheet flapping by the magnetometer, which has been previously observed by Venus Express (Rong et al. 2015). Moreover, the Venusian magnetotail topology (i.e., open, closed or draped field lines) can be investigated by using MPPE electron data and MAG data in conjunction with MHD model results as done at Mars (Luhmann et al. 2015).

Second Venus Flyby The CA closer distance and geometry of the second flyby will bring BepiColombo much closer to the planet and it will cross the bow shock far behind Venus's terminator, where the question about its nature, a shock- or a wave-like structure, can be addressed.

The situation for a quasi-parallel bow shock is quite different. Luhmann (1995), using PVO data studied selected orbits which probed the subsolar point of the magnetosheath and 
found that the waves were mainly linearly polarized and transverse, with no evidence for regularly occurring slow mode like waves structures standing in the flow of the magnetosheath. The second flyby configuration is also very favorable to investigate the nature of plasma turbulence (incompressible vs. compressible) and the spatial evolution of its properties (e.g., scaling laws, intermittency, energy dissipation rate), from the shock to the terminator, as done in the Saturnian (e.g. Hadid et al. 2015), Jovian (Tao et al. 2015), Earth (Huang et al. 2017), Martian (Ruhunusiri et al. 2017) and Hermean systems (Huang et al. 2020). A recent similar study was carried out using Venus Express data in the Venusian magnetosheath (Xiao et al. 2018). However, the potential availability of data from the magnetometers and Mio/PWI (LF-SC and DB-SC), and plasma data from the Mio/MPPE instrument, should allow us to address this problem in a more complete way and over a broader range of MHD and kinetic scales/frequencies. The spacecraft will probably enter the ionosphere during closest approach at an altitude of $\sim 550 \mathrm{~km}$ where BepiColombo is inside the magnetic pileup boundary (MPB). If either PICAM or MSA will have the spacecraft ram vector in their field of view, the instruments would encounter high fluxes of planetary heavy ions. MAG will also observe the horizontal magnetic field lines as IMF penetrates into relatively low altitudes. As shown in Fig. 21 stronger heavy ion fluxes can sometimes not only be observed in the tail region of the planet but also upstream, picked-up and accelerated by the induced electric field. This creates the so-called "ion plume", discussed in, e.g., Dubinin et al. (2011), frequently observed also at Mars by NASA's MAVEN mission (e.g., Brain et al. 2015; Curry et al. 2014).

The second BepiColombo Venus flyby which will scan the flanks of the Venus-Solar wind interaction region. As can be seen in the same figure, total heavy ion fluxes can reach up to $10^{11} \mathrm{~cm}^{-2} \mathrm{~s}^{-1}=10^{7} \mathrm{~cm}^{-2} \mathrm{~s}^{-1}$ at distances below 2 Venus radii. In the magnetosheath there is a high chance to observe planetary ions picked-up by the solar wind flow. Thus, even if the spacecraft will not cross the ionotail during this flyby the chances to observe the planetary ion composition are high. From the point of view of the foreseen level of solar activity, the magnetic shadowing of energetic particles are more likely to be successful during the second flyby. The rapid crossing of the inner region of the induced magnetosphere improves the chances to relate the observed temporal variations with the magnetospheric structures rather than with variations in the ambient magnetic conditions, which can be assessed within minutes of traversing the inner parts of the system. It is likely that solar activity and X-ray irradiance is on average higher during the second flyby, favoring the detection of the SWCX induced X-rays in a geometry looking away from the Sun towards the limb of Venus.

In conclusion, the most significant plasma observations at Venus would be the detection of minor ion species since this was not possible with the limited mass resolution of previous ion spectrometers crossing the Venus plasma environment. Considering that the single-anode geometry factor of Mio/MSA instrument is about $10^{-5} \mathrm{~cm}^{2} \mathrm{sr}$, we expect up to 100 heavy ions per second to be observed by MSA on the second Venus flyby at pericenter distance of about $1.2 R_{V}$. On the first flyby pericenter distance of BepiColombo was $\sim 16770 \mathrm{~km}$ (less than $2 R_{V}$ ). At this distance expected heavy ion fluxes are only about $10^{9.5} \mathrm{~m}^{-2} \mathrm{~s}^{-1}=3.1 \cdot 10^{5} \mathrm{~cm}^{-2} \mathrm{~s}^{-1}$ such that the MSA heavy ion count rate may drop to a few counts per second.

However, during the long crossing in the ionotail mass fluxes are higher and count rates could increase when the spacecraft $-Z$-axis would point more towards Venus. MSA field of view will be restricted to about 5 anodes looking outward from the MOSIF shell. Ideal observing direction will be parallel to the electric field induced by the solar wind, that is perpendicular and towards the Sun-Venus axis. This look direction will also not suffer from saturation by solar wind ions. The expected maximum velocities of pickup ions are twice the 
solar wind velocity. For heavy ions this means an energy of more than $10 \mathrm{keV}$, but the distribution may vary over a broad energy range well separated from solar wind. The PICAM instrument on MPO is optimized to observe ions with energies less than the solar wind energy but with higher fluxes; hence, PICAM is well suited to analyze the ion composition both in the ionotail and during the ionosphere passage in the second flyby. With the high mass resolution of MSA and PICAM, the instruments may deliver the first quantified observation of minor ion escape from Venus as $\mathrm{C}^{+}, \mathrm{N}^{+}, \mathrm{CO}^{+}$or $\mathrm{CO}^{2+}$. Another interesting observation would be the plasma turbulence (at MHD and kinetic scales) and the identification of different plasma wave modes in the different regions of the Venus plasma environment. This can be done primarily using the data from the MPO magnetometer (MAG). Moreover, thanks to the Mio/PWI AC magnetometers (LF-SC and DB-SC), it will be possible for the first time to measure high frequency turbulence waves in the Venusian system (if their amplitudes are large enough to exceed the noise level of the interferences under the limited configuration during the cruise phase). These studies will be further consolidated by the plasma data from MPPE and PICAM.

\subsection{Coordinated Observations}

The Venus atmosphere observation opportunities of BepiColombo (Sect. 5.1.1) will become more comprehensive thanks to the coordinated observation plan with the JAXA's Venus orbiter Akatsuki, and with Earth-based telescopes. These three independent platforms will provide different viewing geometries, so that day and night sides of Venus will be captured simultaneously. Previously reported phenomena, such as thermal tides (Kouyama et al. 2019) and local time dependent $\mathrm{SO}_{2}$ gas distributions (Encrenaz et al. 2019; Marcq et al. 2020), will be better understood in terms of spatial and temporal variabilities.

BepiColombo's Venus atmosphere observations will be performed by the UV spectrometer PHEBUS, and the mid-IR radiometer and spectrometer MERTIS. The coordinated observations at the overlapped UV wavelength will allow cross validation of measured UV data (Lee et al. 2019), between PHEBUS and a UV Imager onboard Akatsuki (UVI, Yamazaki et al. 2018). That at the overlapped mid-IR wavelength will allow a comparison between close-up spectral features of the cloud top level atmosphere obtained by MERTIS and global thermal structure acquired by the Longwave Infrared Camera onboard Akatsuki (LIR, Taguchi et al. 2007). A similar wavelength will be used also from Earth-based coordinated observations, allowing detection of specific targets in the Venus atmosphere. For example, UV to visible range wind vectors derived from Akatsuki and ground-based observations can be compared with thermal winds derived from the temperature map of MERTIS (Peralta et al. 2017; Machado et al. 2017); $\mathrm{SO}_{2}$ abundance measurements can be supported with UV and IR observations from Akatsuki and the ground-based observations too (Jessup et al. 2015; Encrenaz et al. 2019). When short time scale features of Venus will be captured during approach and flybys of BepiColombo, Akatsuki will continuously monitor Venus, and ground-based telescope will expand the spatial and spectral coverages of the observations. These planned comparisons will contribute to a fundamental understanding of Venusian clouds, chemistry, dynamics, radiative energy balance (Sánchez-Lavega et al. 2017; Limaye et al. 2018; Titov et al. 2018; Marcq et al. 2020), and climate and decadal scale albedo trends (Lee et al. 2019).

First Venus Flyby Akatsuki has been operated since December 2015 (Nakamura et al. 2016), and its first extended operation phase is currently scheduled until March 2021. The Akatsuki UVI and LIR cameras are planned to perform Venus observations in coordination with PHEBUS and MERTIS, respectively. 


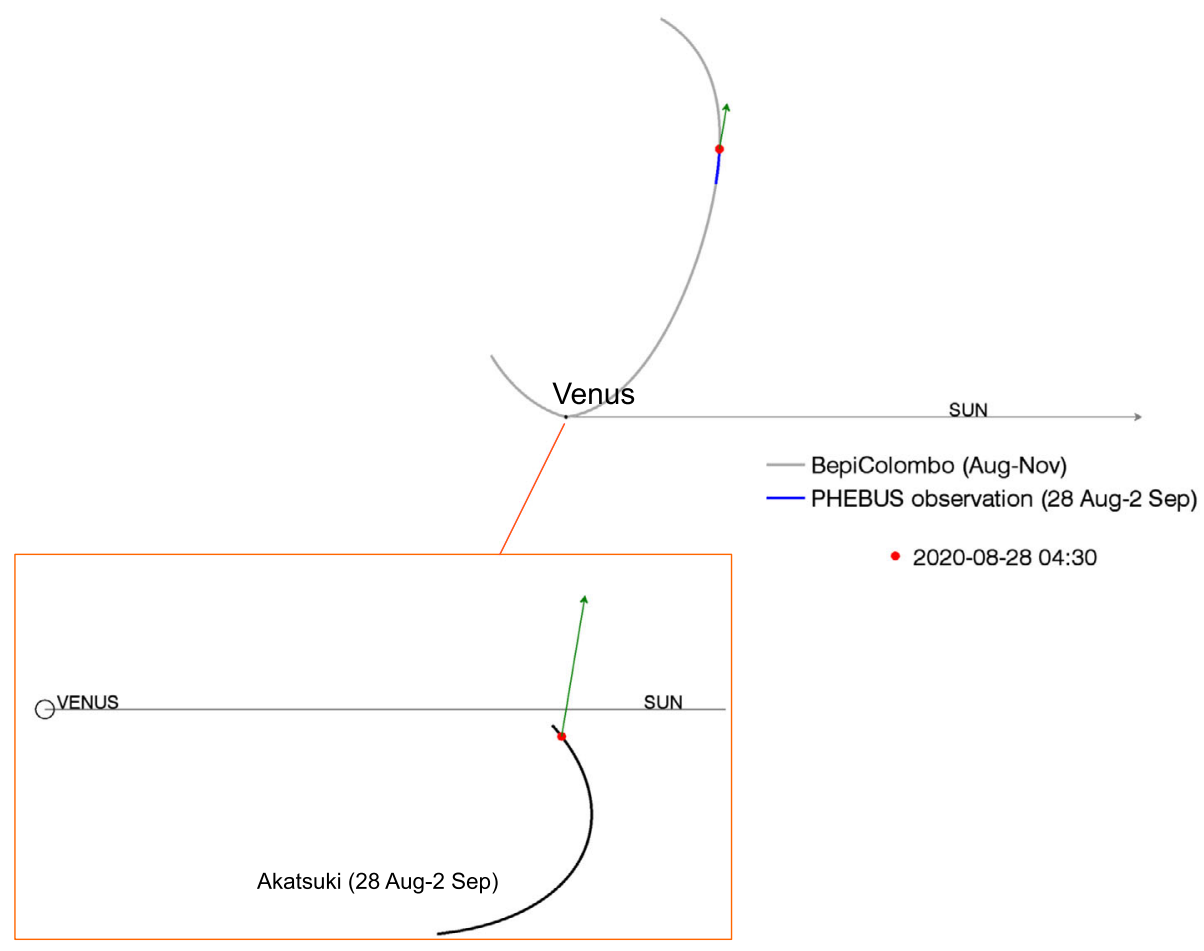

Fig. 22 Relative locations of the Sun, Venus, BepiColombo (blue), and Akatsuki (black) on 28 Aug-2 Sep 2020. The $+X$ is assigned to the Sun. The grey curve is the trajectory of BepiColombo from August to November 2020. Red dots are the locations of BepiColombo and Akatsuki on 28 August 2020. The green arrows indicate the direction towards the Earth
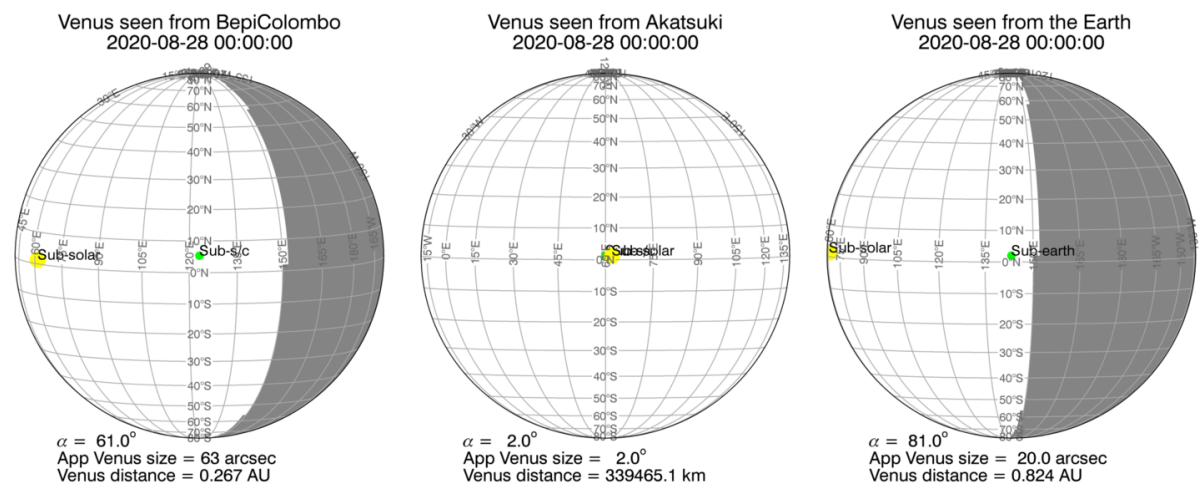

Fig. 23 Venus as seen from BepiColombo (left), Akatsuki (center), and Earth (right) on 28 August 2020. The size of Venus is not scaled, but the apparent sizes in degree or arcsec are written in the bottom. Solar phase angle $(\alpha$, degree) and the distance to the center of Venus are also indicated

UVI has two filters centered at 283 and $365 \mathrm{~nm}$ (Yamazaki et al. 2018): it monitored the Venus dayside, when PHEBUS performed dayside faraway observation on 28 Aug-2 Sep (see Figs. 22 and 23, and Sect. 5.1.1). The dayside was captured at three different solar phase 
Fig. 24 Relative locations of the Sun, Venus, BepiColombo (blue), and Akatsuki (black) on 10-20 October 2020. The $+X$ is assigned to the Sun. Red dots are the locations of BepiColombo and Akatsuki at the time of the 1st Venus flyby CA (03:58 UT 15 October 2020). Green arrow indicates the direction of the Earth

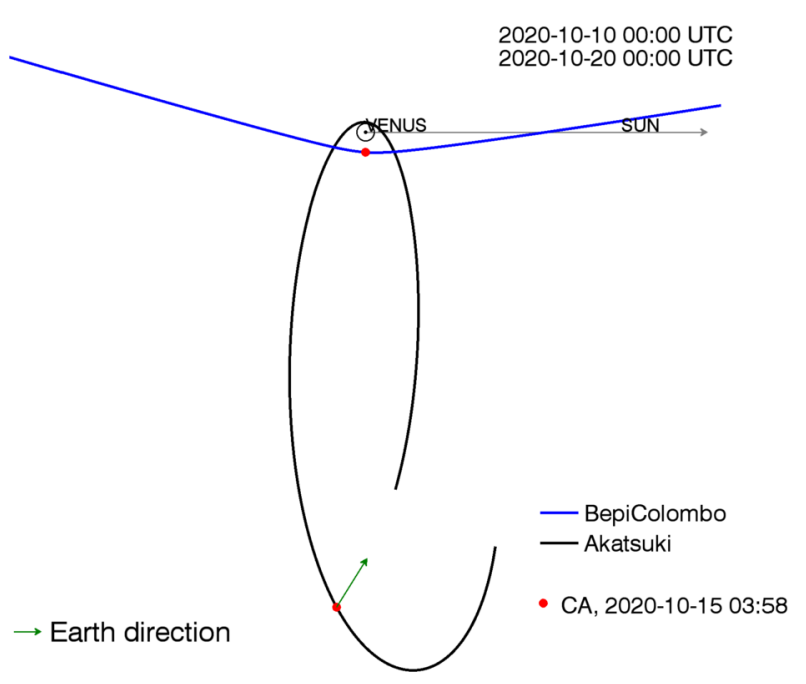

angles, including the one from the Earth (Fig. 23) on 20 Aug-3 Sep: this fact allowed a wide range of solar phase angles to be observed at the same time (for the first time ever). The coordinated faraway disk-integrated albedo observations performed are important to study the long-term variations of Venus's UV albedo (Lee et al. 2019) and to validate the observed radiance. These coordinated observations will be also used to retrieve the absorption spectrum of the "unknown absorber" in the Venusian clouds in the EUV-FUV range, by mean of the simultaneous $365 \mathrm{~nm}$ imaging of UVI, which reveals the most pronounced absorption of the "unknown absorber" (Pérez-Hoyos et al. 2018; Marcq et al. 2020). In addition, these faraway broad wavelength observations will extend the spectral coverage of Venus analysis as an exoplanet (Lee et al. 2020), which is a valuable reference to characterize Venus-like cloudy exoplanets.

LIR's filter is centered at $10 \mu \mathrm{m}(8-12 \mu \mathrm{m}$ bandwidth) (Taguchi et al. 2007). Recent discovery of planetary scale waves on the cloud level induced by the surface mountains were discovered (Fukuhara et al. 2017). Clear local time dependence of the waves had been reported (Kouyama et al. 2017), and the interaction between the surface and atmosphere implies a possibility of slowing down of solid body rotation rate (Navarro et al. 2018). At the time of BepiColombo's CA, LIR was monitoring the entire globe near the apocenter of Akatsuki's orbit (Figs. 24 and 25). Therefore, global scale thermal structures at the cloud top across the evening terminator could be obtained by LIR. Contemporary MERTIS's close-up observations over the same location can provide insights of the planet's atmosphere from its spectral analysis.

During the first flyby closest approach, Earth-based facilities have imaged the Venus' morning side, which was partially overlapped with MERTIS observations (late morning to noon area, see Fig. 25), but not by Akatsuki. Therefore, Earth-based observations can extend local time coverage to understand instantaneous global thermal tide, in addition to its long-term average (Kouyama et al. 2019). Also, $\mathrm{SO}_{2}$ gas abundances can be compared between the global measurements from the Earth and the close-up measurement from MERTIS (Encrenaz et al. 2019). While a cloud-tracking method using UVI images can provide wind vectors over the afternoon side, Earth-based imaging and Doppler shift measurements can provide those over the morning side (Sánchez-Lavega et al. 2016; Machado et al. 2017). 

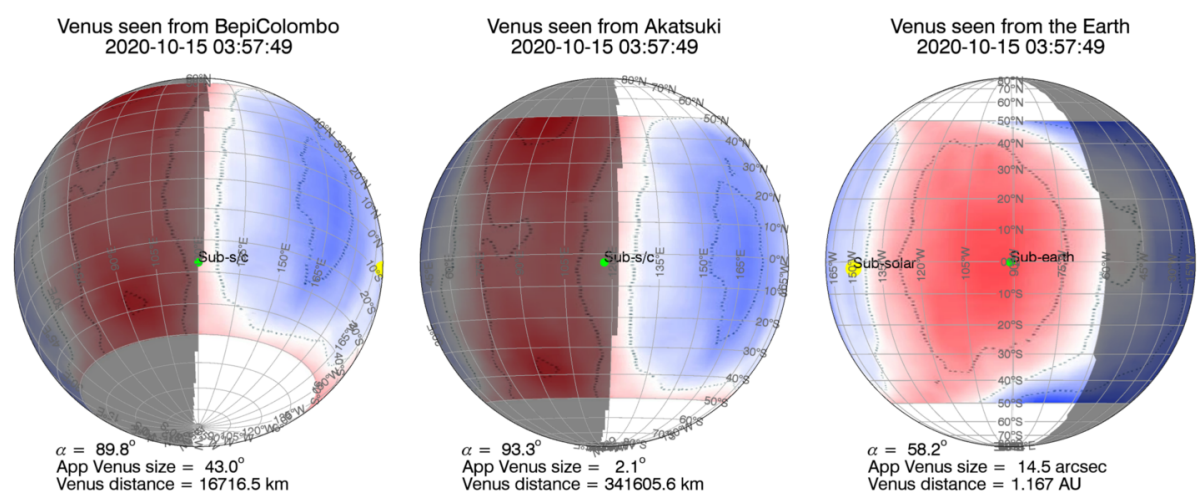

Fig. 25 Venus as seen from BepiColombo (left), Akatsuki (center), and Earth (right) at the time of the 1st Venus flyby CA (03:58 UT 15 October 2020). The size of Venus is not scaled, but the apparent sizes in degree or arcsec are written at the bottom. Solar phase angle ( $\alpha$, degree) and the distance to the center of Venus are also indicated. The background Venus image is LIR's observed mean thermal tide, taken from Kouyama et al. (2019)

Many professional astronomers, and even amateur Venus observers, contributed to this effort (Garate-Lopez et al. 2020).

Second Venus Flyby If the Akatsuki mission extension will be determined, the coordinated observations with BepiColombo will be performed again during the 2nd flyby. In August 2021 flyby, BepiColombo and Earth-based observations, on the other hand, will observe the same area of Venus. This is a condition favourable to compare different observational target over the same area simultaneously, allowing comparison for different altitudes within the atmosphere using different wavelengths.

In addition to coordinated observations with Akatsuki, the ESA Solar Orbiter spacecraft will cross Venus during the same days, and have its own flyby at Venus on August 9th, 1 day before BepiColombo.

Solar Orbiter is an ESA spacecraft dedicated to the study of the Sun and of the inner heliosphere by mean of remote and in situ instrumentation. Solar Orbiter in situ instruments are planned to be operative during Venus flybys, and will contribute to coordinated observations by investigating the variability of magnetic field and ionized environment, by mean of measurements with the following instrumentation: Energetic Particle Detector (EPD), Radio and Plasma Waves (RPW), Solar Wind plasma Analyser (SWA) and the magnetometer (MAG). These instruments will coordinate with the BepiColombo sensors measuring electromagnetic field, plasma waves, and ions and electron populations at different energies (see Sect. 5.1.2 for details). Hence, during the second flyby, not only the atmosphere may be studied with more details thanks to the much lower altitude of BepiColombo at CA, but also the Venus' ionized environment and the planetary interactions with the solar wind can be better investigated, thanks to the very close (in time) flyby of a spacecraft devoted to the study of the inner heliosphere.

\section{Mercury Flybys}

Before entering into orbit around Mercury, BepiColombo will perform six flybys at Mercury that will allow progressive modifications to the interplanetary trajectory. The flybys will take place in 2021, 2022, 2023, 2024 (twice), and at the beginning of 2025. 
Table 3 Mercury flybys dates and their main characteristics. No eclipse will occur during Flybys 4 and 5; no occultation during Flybys 1 and 5 (then, classified as N/A in the table)

\begin{tabular}{|c|c|c|c|c|c|c|}
\hline & \multicolumn{6}{|c|}{ Mercury flybys } \\
\hline & $\# 1$ & $\# 2$ & \#3 & $\# 4$ & \#5 & \#6 \\
\hline Date & 1 Oct 2021 & 23 Jun 2022 & 20 Jun 2023 & 5 Sep 2024 & 2 Dec 2024 & 9 Jan 2025 \\
\hline $\begin{array}{l}\text { Closest Approach } \\
{[\mathrm{km}]}\end{array}$ & 200 & 200 & 200 & 200 & 40000 & 393 \\
\hline $\begin{array}{l}\text { Electric propulsion } \\
\text { end }\end{array}$ & $C A-38 d$ & $\mathrm{CA}-39 \mathrm{~d}$ & $\mathrm{CA}-44 \mathrm{~d}$ & $\mathrm{CA}-85 \mathrm{~d}$ & $\mathrm{CA}-38 \mathrm{~d}$ & $\mathrm{CA}-75 \mathrm{~d}$ \\
\hline $\begin{array}{l}\text { Electric propulsion } \\
\text { start }\end{array}$ & $\mathrm{CA}+7 \mathrm{~d}$ & $\mathrm{CA}+7 \mathrm{~d}$ & $\mathrm{CA}+51 \mathrm{~d}$ & $\mathrm{CA}+7 \mathrm{~d}$ & $\mathrm{CA}+45 \mathrm{~d}$ & $\mathrm{CA}+8 \mathrm{~d}$ \\
\hline Sun distance $[\mathrm{AU}]$ & 0.38 & 0.38 & 0.33 & 0.32 & 0.32 & 0.45 \\
\hline $\begin{array}{l}\text { True Anomaly } \\
\text { Angle [deg] }\end{array}$ & 260 & 263 & 312 & 331 & 331 & 150 \\
\hline $\begin{array}{l}\text { Eclipse duration } \\
\text { [mn] }\end{array}$ & 13 & 18 & 15 & N/A & N/A & 24 \\
\hline $\begin{array}{l}\text { Occultation } \\
\text { duration [mn] }\end{array}$ & N/A & 12 & 15 & 20 & N/A & 9 \\
\hline $\begin{array}{l}\text { Sun-Earth-MCS } \\
\text { angle [deg] }\end{array}$ & 15 & 22 & 13 & 18 & 9 & 18 \\
\hline $\begin{array}{l}\text { Earth distance } \\
{[\mathrm{AU}]}\end{array}$ & 0.69 & 0.96 & 1.22 & 0.94 & 0.70 & 1.27 \\
\hline
\end{tabular}

Table 3 provides a summary of the Mercury flybys main characteristics and Fig. 26 the spacecraft angular distances from the Sun, Mercury, and the Earth in the 48 hours around CA. Regarding attitude constraints, at a Sun distance $<0.7 \mathrm{AU}$, it is possible to offset the Sun direction in the spacecraft composite $+Y Z$ plan, in the range of $+14^{\circ}$ to $-9.1^{\circ}$ (see Table 9 in the Appendix). Eclipses will occur during Flyby 1, 2, 4 and 6, and the closest approaches will take place in the middle of eclipse and last 13, 18, 15 and 24 minutes, respectively. In eclipse, the link between MTM and MPO is cut; this is the reason why each module is provided on its own battery. The MPO battery will be charged to $100 \%$ for the Mercury flybys with eclipses. Earth occultations will occur in the Flyby 2, 3, 4, and 6 and will last 12,15, 20, and 9 minutes, respectively.

Figure 27 (upper panel) shows the flybys geometries in the Mercury Solar Magnetic (MSM) frame, $X_{M S M}-\rho_{X}$ plane where $\rho_{X}$ is:

$$
\rho_{X}=\sqrt{Y_{M S M}^{2}+Z_{M S M}^{2}}
$$

All close-in flybys (except for Flyby 4) have pericenters at the nightside of the planet. Flybys 1,2 and 3 are roughly equatorial. Flybys 4 and 6 are polar. Figure 27 (bottom right) shows the flyby positions as seen from the Sun ( $Y-Z$ plane, MSM frame). Figure 27 (bottom left) shows BepiColombo Mercury flyby trajectories in the Mercury Solar Orbital (MSO) frame, $Z-\rho_{Z}$ plane, compared to the MESSENGER flybys (dashed lines) and during its orbital mission phase (grey area).

All Mercury flybys (perhaps with the exception of Flyby 5) will be close enough to the planet to allow to take a wide range of novel and relevant measurements of the Hermean magnetosphere, exosphere, surface and interior. 

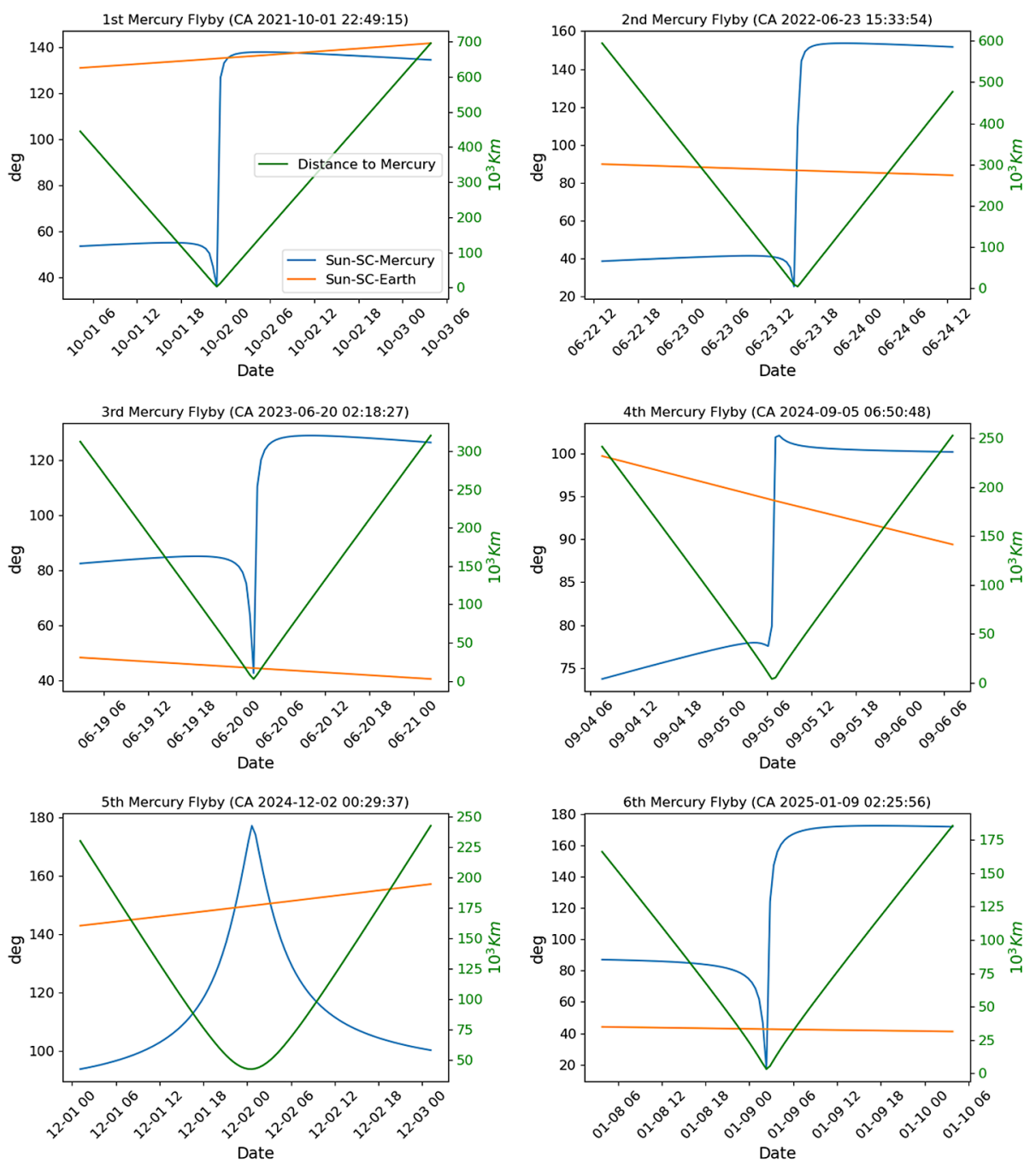

Fig. 26 BepiColombo linear and angular distances during the six Mercury flybys: to the planet (green), to Sun and Mercury (blue), to Sun and Earth (orange)

\subsection{Scientific Objectives}

Full scientific objectives of Mercury investigations will be addressed in the nominal phase of BepiColombo, starting in 2026, when the two spacecraft will be orbiting around the planet in its final configuration. Despite the limitations in the operative instruments and performances, the 6 flybys will surely help to provide first BepiColombo data of the Hermean environment already five years in advance. In the following, some highlights of achievable scientific objectives are described.

\subsubsection{Magnetosphere and Plasma Environment}

Mercury is a natural laboratory for the comparative study of magnetospheric dynamics (Slavin et al. 2019). Mercury's internal magnetic field (190 nT $R_{M}{ }^{3}$ ) is much weaker than 

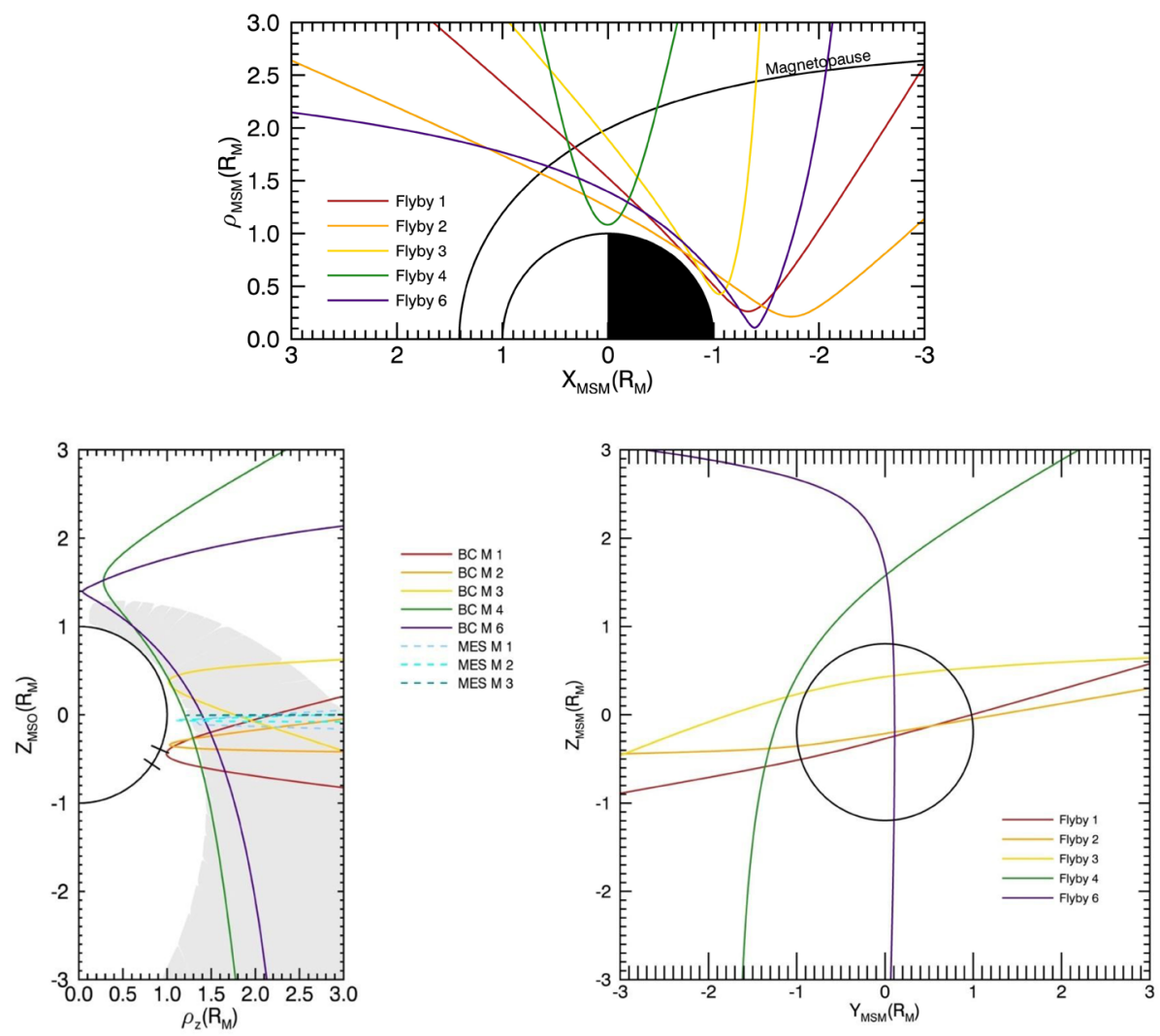

Fig. 27 Top: the flyby trajectories of BepiColombo at Mercury projected onto the MSM frame, $X-\rho(x)$ plane. Black line is both for the planet profile and the magnetopause average position (Korth et al. 2017). Bottom right: BepiColombo Mercury flybys as seen from the Sun ( $Y-Z$ plane, MSM frame). Bottom left: BepiColombo Mercury flyby trajectories in the MSO frame, $Z-\rho(z)$ plane, compared to the MESSENGER flybys (dashed lines). The light grey area indicates the satellite positions of MESSENGER in its orbital mission phase. The two dashes on the planetary profile mark the area studied by Winslow et al. (2014) using proton reflectometry data

the Earth's (30400 nT $R_{M}{ }^{3}$ ) (Anderson et al. 2012). Despite the weakness of the internal magnetic field of Mercury, the interaction with the solar wind leads to the formation of the bow shock and magnetopause boundaries as at the Earth. At Mercury heliocentric distance the solar wind density is 10 times higher than at the Earth, and the IMF intensity is about 10 times higher, while its velocity remains the same. As a consequence, the solar wind dynamic pressure at Mercury is on the average $\sim 10$ times larger. The high solar wind dynamic pressure, IMF and radiation environments at Mercury may mimic what exoplanets experience in close-in habitable zones of M dwarfs (e.g., Dong et al. 2018a; Airapetian et al. 2020), thus the investigation of solar wind interaction with Mercury may offer fresh insights concerning the exoplanetary habitability too. Meanwhile, the extreme solar wind conditions result in substantial differences between the Hermean and the Terrestrial magnetospheres interactions with the solar wind. The Hermean shock is much weaker, as the solar wind features lower Mach number and plasma $\beta$ (the ratio between plasma pressure and magnetic pressure) at Mercury than at Earth. Consequently, the stream of ions back- 


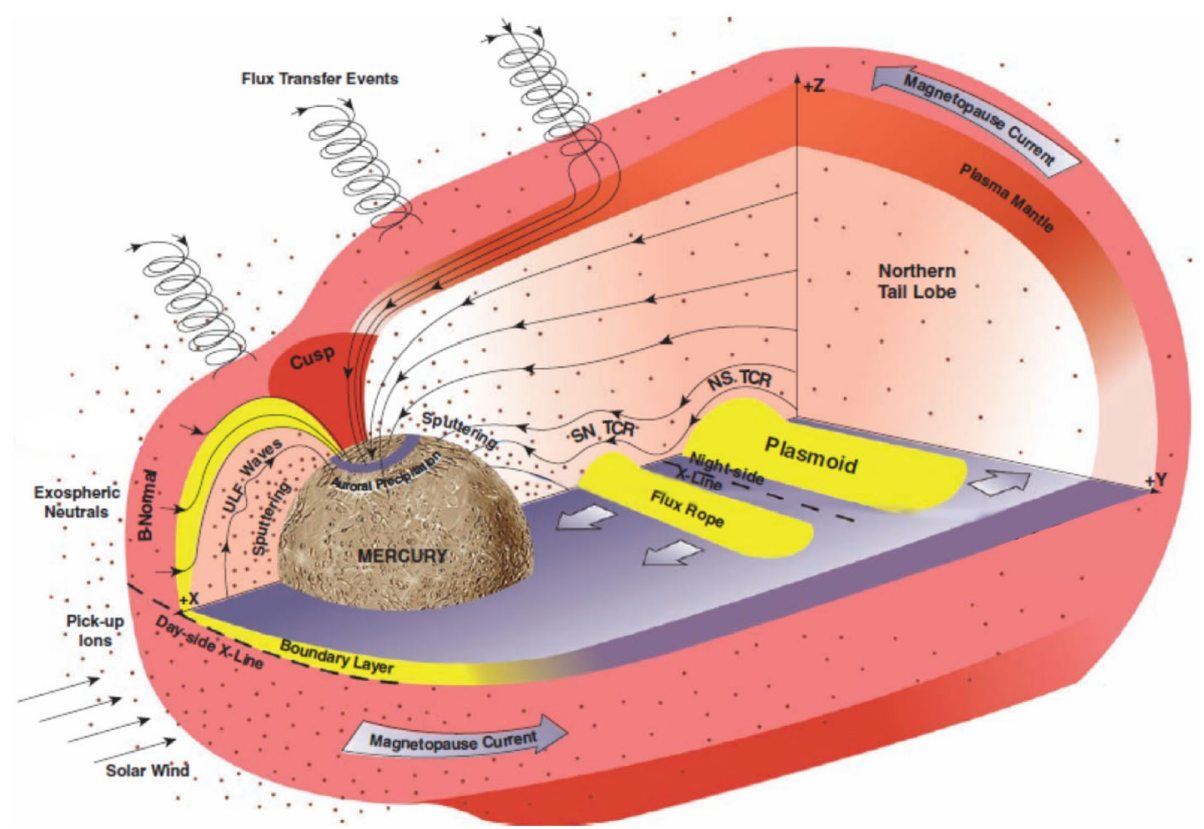

Fig. 28 Sketch of Mercury magnetosphere in active conditions, as seen from MESSENGER during its second flyby on 6 October 2008, showing the possible magnetospheric phenomena that could be observed during BepiColombo flybys investigations (adapted from Slavin et al. 2009)

streaming into the incoming solar wind is weaker and the foreshock volume is smaller and structures have less time to evolve and steepen before they are carried to the shock (Le et al. 2013). The foreshock is more often located upstream of the subsolar shock, though the Parker spiral IMF angle is typically small (about 20 ${ }^{\circ}$ (e.g. Slavin and Holzer 1981). Low $\beta$ conditions are enhanced due to the extended Plasma Depletion Layer (PDL) that is commonly present upstream of the Hermean magnetopause (Gershman et al. 2013) for almost all IMF orientations. PDL contributes more significantly to the high reconnection rate at Mercury in comparison to Earth, leading to rapid successions of flux transfer events (FTEs) at the magnetopause (Slavin et al. 2012b; Imber et al. 2014; Imber and Slavin 2017; Zhong et al. 2020b), magnetotail flux ropes and plasmoids (Slavin et al. 2009, 2012a; DiBraccio et al. 2015; Smith et al. 2017; Zhong et al. 2019), and dipolarizations or reconnection fronts (Sundberg et al. 2012; Zhong et al. 2018), as observed during the second Mercury flyby by MESSENGER (see Fig. 28).

Mercury Flybys Investigations Despite the recent MESSENGER observations, a full understanding of the reconnection and particles acceleration mechanisms at Mercury is still not achieved. In this sense, BepiColombo double spacecraft mission will greatly improve our understanding of the dynamics of the small magnetosphere and of plasma environment of Mercury, providing - for the first time - simultaneous multi-spacecraft observations during the nominal mission phase (Milillo et al. 2020, this journal). Nevertheless, each one of the six Mercury flybys, though with BepiColombo spacecraft in stacked configuration, will gather cross-sectional measurements of the environment, by crossing the foreshock, the bow shock, the magnetosheath, magnetopause boundaries and different magnetospheric regions. 
Most of these flybys will cross the low-latitudes magnetospheric flanks that are less covered by the polar orbits of the two BepiColombo spacecraft (see Fig. 27).

Several scientific issues have been identified during BepiColombo Mercury flybys. The instruments that can be operated for the investigations of these regions are:

- MPO-MAG (fully operative) providing magnetic field at high time resolution,

- MGF and PWI providing magnetic field (at less time resolution since the booms are not deployed),

- the sensors of SERENA and of MPPE that will provide ion and electron full energy but partial angular distributions (due to their position in the stacked configuration),

- SIXS-P and BERM that will detect the high energy protons and electrons,

- and SIXS-X providing X-rays (see Table 1).

As shown in Fig. 27, during each of the Mercury flybys (except for Flyby 5), the spacecraft will cross the bowshock providing a good opportunity to measure the ion waves at the foreshock (with PWI experiment and the magnetometers MAG and MGF). Also the close-in flybys will be an opportunity to investigate the magnetopause reconnection and the dynamics and evolution of FTEs (with the measurements of magnetic field (MAG and MGF), electrons and ions (MPPE and SERENA). During the magnetospheric crossing SIXS-X and SIXS-P together with the BERM radiation monitor will provide information of possible solar events, like Solar Energetic Particles or Flares. These measurements, coupled to magnetic field and particle data, will help our understanding of Mercury's dynamic magnetosphere in relation to the Sun activity.

As already noticed, all close-in flybys (except for Flyby 4) have pericenters at the nightside of the planet, and Flybys 1, 2, and 3 are nearly equatorial. As a consequence, Flybys 1, 2 , and 3 can be used to study dawn-to-dusk asymmetries in the tail, down-tail evolution of plasma sheet, lobes, and the nightside magnetotail radius from direct encounters (e.g. Slavin et al. 2010). These flybys are also uniquely designed for the study of the low-latitude boundaries, including the equatorial diamagnetic depressions expected on the dawn side (Müller et al. 2012), nightside reconnection (including x-line location (e.g. Slavin et al. 2009), reconnection dynamics (Zhong et al. 2018, 2020a), potentially substorm loading-unloading phases (e.g. Imber and Slavin 2017), and Kelvin-Helmholtz vortices at the magnetopause. Such measurements had been challenging from high-latitude MESSENGER orbits and will be challenging also for BepiColombo orbits during the nominal mission.

Flybys 4 and 6 are nearly polar. During Flyby 4, when approaching the Hermean geographical northern pole, quasi-steady "Region 1" field-aligned currents is expected (Anderson et al. 2014): these currents flow along magnetic field lines from the magnetopause region at dawn, down to the planet and emerge again on the dusk side flowing again towards the magnetopause. A big open issue is whether these currents are closed in between, either by the planet itself and/or by the magnetosphere. This depends on the available electrical conductivity, which has not been yet fully constrained.

Flyby 4 could also cross the northern cusp (e.g. He et al. 2017; Zhong et al. 2015) which provides an opening window for magnetosheath plasma to the magnetosphere.

Flyby 6 has the closest approach in the nightside high latitudes, and it could be used to study a magnetic Z-profile of the Hermean magnetotail to be compared with the average profile presently known from MESSENGER data (e.g. Rong et al. 2018). On the contrary, "Region 2" field-aligned current systems associated with substorm current wedges have not been observed so far with MESSENGER data (e.g. Glassmeier 2000; Poh et al. 2017).

Finally, the BepiColombo Flybys 4 and 6 could provide low-altitude measurements of magnetic field, electrons, and ions in the nightside, giving a chance to detect these signatures for the first time ever. 


\subsubsection{Intrinsic and Crustal Magnetic Field}

The planet's weak internal global magnetic field (Ness et al. 1975), with an intensity of about only $1 \%$ of that of the Earth was discovered by the NASA mission Mariner 10 in the 1970s. The MESSENGER spacecraft, during its orbit around the planet in the years 2011-2015, characterized the magnetic field widely (Anderson et al. 2011), but not entirely. Due to MESSENGER's eccentric orbit, only data from the northern hemisphere close to the planet are available. The lack of information in overall the southern hemisphere makes the task of modeling the internal magnetic field very difficult and leads to correlations between the Gauss coefficients describing the magnetic field multipoles (see discussions in Iess et al. 2021 and in Heyner et al. 2021, this journal). The exact determination of the internal magnetic field is further complicated at Mercury, because the reconnection and induction change has very significant impacts on the amount of magnetic flux above the surface of Mercury (Jia et al. 2019; Dong et al. 2019). As a result Mercury's dipolar magnetic moment varies by up to at least $25 \%$ during solar wind transients (especially CMEs) (Slavin et al. 2019). Very low altitude magnetic field measurements obtainable, especially during the flybys, will allow a better determination of the dipole moment. The models of the Hermean core magnetic field have been proposed when studying the MESSENGER magnetometer data can be summarized as:

- a global core magnetic field model using the magnetic equator crossing positions as an initial constraint (Anderson et al. 2012);

- a regional model using a discrete regional method (Oliveira et al. 2015);

- a regional model using a continuous regional method (Thébault et al. 2018).

Two common features emerge in all models: the strong axisymmetry of the core field, and the location of the magnetic equator in the northern hemisphere. Only rough constraints could be established for the secular variation between Mariner 10 flybys and the MESSENGER orbital phase (Philpott et al. 2014) leaving the possibility that the field could not change within the 40 years that separated the 2 missions.

For latitudes between $35^{\circ} \mathrm{N}$ and $75^{\circ} \mathrm{N}$, present day models of the Hermean crustal magnetic field were calculated by using the MESSENGER low altitude campaign data (Hood 2016; Hood et al. 2018). It was revealed that magnetic anomalies are distributed heterogeneously over the planet's surface, where the strongest anomaly ( $\sim 14 \mathrm{nT}$ at $40 \mathrm{~km}$ altitude) is related with the Caloris basin.

Mercury Flybys Investigations In 2021, six years after the end of MESSENGER's orbital mission, the first BepiColombo flybys to Mercury will allow us to look for hints of secular variation in the dynamo generated field (if they exist) and to confirm the asymmetry in the magnetic field. This would further constrain the type of dynamo operating in the Hermean core. Figure 27 (bottom left) displays the trajectories of the six BepiColombo flybys compared to the three MESSENGER flybys in the $Z-\rho(z)$ plane in the MSO frame.

The MESSENGER positions (Fig. 27, light grey area) from its orbital mission phase are shown for comparison. It can be noticed that, at the pericenters of Flybys 1 and 2, BepiColombo will move close to the planet in a region not previously explored by MESSENGER and will be able to sample the Hermean magnetic field in the southern hemisphere. Hence, the Hermean internal magnetic dynamo models established with MESSENGER magnetic field data (e.g. Wicht and Heyner 2014) can be critically tested. Also, these new measurements can be compared to the study by Winslow et al. (2014), who used the proton reflectometry method to assess the surface magnetic field at the southern hemisphere for the first time. 

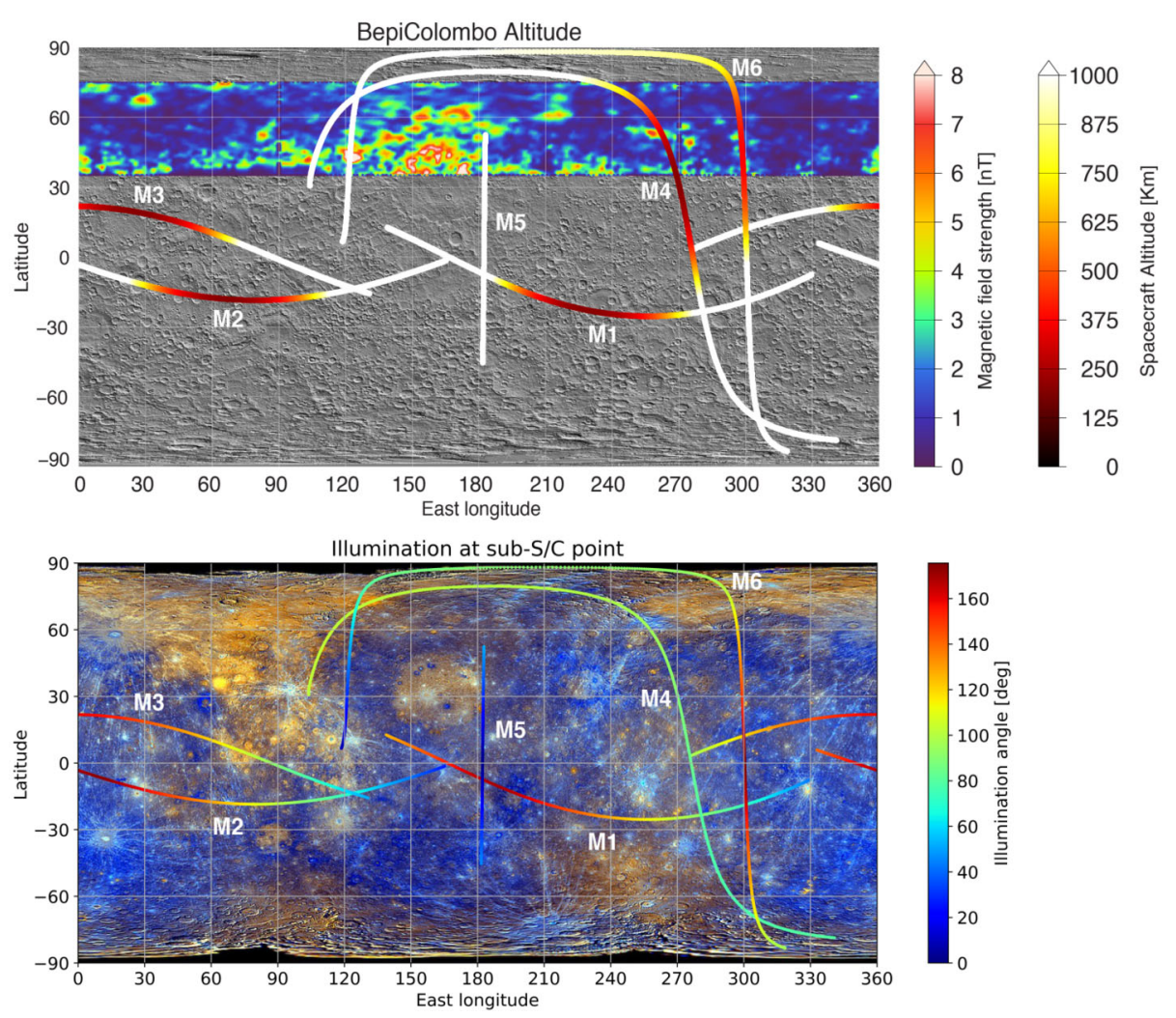

Fig. 29 BepiColombo Mercury Flybys (here named M1 to M6) footprints on the Hermean surface. (top) BepiColombo sub-spacecraft altitude (coloured tracks), overlapping the topography (grey scale). The coloured map represents the crustal magnetic field strength measured by the MESSENGER magnetometer at $40 \mathrm{~km}$ altitude over the northern mid-latitudes (Hood et al. 2018). (bottom) BepiColombo sub-spacecraft illumination angle (coloured tracks) superimposed on the MDIS enhanced colour global mosaic. Illumination angles below $90^{\circ}$ correspond to the dayside, and the closest approach of each flyby (below $1000 \mathrm{~km}$ ) will mostly occur on the nightside. The maximum altitude represented here is $60000 \mathrm{~km}$. The topography and enhanced colour global mosaics is gathered from USGS

More challenging but still possible, will be the measurement of crustal magnetic fields during the flybys, in the case of stronger anomalies than the ones measured in the northern hemisphere by MESSENGER. Figure 29 displays the projected ground track of the BepiColombo flybys on top of a topographic surface map. The crustal magnetic field at $40 \mathrm{~km}$ altitude above the ground is depicted as a color overlay in a northern mid-latitude band. In particular, BepiColombo flybys 4 and 6 will pass over the crustal magnetic field regions mapped by MESSENGER; measurements during these flybys could be used to test the crustal magnetization analysis by Oliveira et al. (2019), because these flybys have appropriate pericenters altitudes. Magnetic field data from the remaining flybys could constrain crustal fields at regions not probed so far by MESSENGER, years before the beginning of the orbital science operations in 2026. Hence, detailed sampling of the planetary magnetic field by MPO-MAG, with sampling $\geq 1 \mathrm{~Hz}$, should be planned to give crucial information to constrain both core field and crustal field models, and thereby shed more light on the dynamo processes operating in the deep interior. 


\subsubsection{Other Science Goals}

Exosphere Also the study of the exosphere will benefit of the possible investigation during the flybys. The flybys geometries will allow detection and precise measurements of the exospheric components even at altitudes well below the final orbit of MPO during nominal phase (200 km to be compared to minimum MPO cruise altitude of $\sim 450 \mathrm{~km}$ ). Hence, it will be possible to derive vertical density profiles of different exospheric species already detected ( $\mathrm{Na}, \mathrm{Ca}, \mathrm{K}, \mathrm{Mg}, \mathrm{H}, \mathrm{He}, \mathrm{Fe}, \mathrm{Al}, \mathrm{Mn}$ ) but also to detect, for the first time, new species not yet discovered. The equatorial flybys (1,2 and 3) can be used to investigate the dawn-dusk asymmetries, while polar ones (4 and 6) to monitor North-South asymmetries. The elongated trajectories of the flybys can allow scanning the elongated exospheric tail at distances up to few tens of Mercury radii and provide information on the acceleration processes, escape rates, and dynamics occurring in the tail. Integrated dayside and nightside exosphere emission and simultaneous imaging of different regions of the exosphere (as high latitude and equatorial, for example), can be additional measurements that will be worth performing during flyby.

All these measurements can be performed by PHEBUS (for details, see Quémerais et al. 2020). The environment sensors onboard Mio and MPO (primarily, MPPE and SERENA) may provide additional information on the ionized populations counterpart (at different energies) along the flybys trajectory paths, and investigate the link with the intrinsic magnetosphere and the interplanetary magnetic field to build a comprehensive view of the Mercury environment.

Dust The dust monitor MDM, if operating and with the reduced field of view in favourable configuration, can make measurements of the micrometeoroid population of the region crossed by the MCS during flybys, and investigate the spatial variability of the dust along the Mercury orbit (Kobayashi et al. 2020). Note that true anomaly angles of Mercury during flybys will be ranging from $\sim 150^{\circ}$ to $\sim 330^{\circ}$ (see Table 3 ), providing a wide range of heliocentric position to be investigated.

Surface Surface measurements will also benefit of the flyby campaign of observations. They will primarily come from MERTIS and MGNS.

MERTIS measurements will provide a first imaging of the Mercury global surface composition in the 7-14 $\mu \mathrm{m}$ spectral range, a region not covered by previous missions. In particular, feldspars can be spectrally identified and characterized, by means of several diagnostic spectral features (i.e. the Christiansen frequency, the Reststrahlen bands, and the transparency feature). In the thermal infrared range at wavelengths longer than $7 \mu \mathrm{m}$, spectral signatures in silicates result from characteristic fundamental $\mathrm{Si}-\mathrm{O}$ vibrations. Therefore $\mathrm{FeO}$ - and $\mathrm{TiO}_{2}$-free silicates (e.g., feldspars, Fe-free pyroxenes and Fe-free olivines), which are almost undetectable in the visible-NIR region, can be identified already during the flybys observation. Finally, MERTIS will implement tests for the proper instrumental set-up and calibration to be used during the nominal mission.

The MGNS measurements will make relevant tests for proper observation of the surface composition and mapping when in nominal phase around the planet. The major objectives for these measurements are for calibration purposes. The flux of the planetary emission is expected to increase with increasing solid angle of the planet (with the maximum value at pericenter), while the background of gamma-rays and neutrons of the spacecraft due to bombardment by Galactic Cosmic Rays will correspondingly decrease. By analyzing opposite variations of gamma-ray nuclear lines of different elements (like $\mathrm{Si}, \mathrm{Al}, \mathrm{Mg}$, etc.), it will 
allow discriminating between lines emitted by the planetary surface and lines emitted by the metallic structure of the spacecraft itself. The MGNS data of flybys will also allow making the direct comparison of the local background data (in terms of gamma rays and neutrons) for two quite different configurations of the spacecraft - the stacked configuration MCS and MPO - while they are at the same positions above the planet. In particular, also the spacecraft crossing of the Hermean magnetosphere boundaries with its high density of ionized particles may contribute to the counts of the MGNS detectors; hence, it should be known in advance before nominal mapping of Mercury surface to remove it (but it will be important information for the magnetospheric studies). Finally, the first proper data of the Hermean surface composition may also be obtained during the flybys (Mitrofanov et al. 2021, this journal).

\subsection{Coordinated Observations}

As for the case of Venus, coordinated Earth-based observations of Mercury during flybys (and then during the nominal mission) will be a valid support for in-situ measurements for exospheric and magnetospheric science. Many discoveries and studies of the exosphere of Mercury during the last three decades were done by means of Earth-based observations. They have also proven that the exosphere is very responsive to the surrounding environment, and in particular to the solar activity, even directly or through the mediation of the magnetosphere.

Coordinated observations of the exosphere are possible from the ground when Mercury has ample solar elongation angles, typically $>20^{\circ}$. Such efforts can be optimally timed utilizing solar telescopes to measure bright sodium emission at 5890 and $5896 \AA$ occurring during the day from the exosphere. Sodium exosphere (discovered from the Earth in 1985, Potter and Morgan 1985) shows distinctive features, still not fully understood. In particular, the sodium exospheric shows typical 2-peaks intensity at high latitudes, and a highly variable dynamics over time scales ranging from minutes (Massetti et al. 2017) up to the planetary orbit time (Cassidy et al. 2015).

Historically, the solar telescope McMath-Pierce performed a long series of Na exospheric observations starting from the ' 80 s, evidencing the two peak intensity pattern for the first time, as well as dawn-dusk asymmetry.

More recently, the solar telescope THEMIS has proven to be a particularly valuable facility for performing daily observations of the exosphere above the disk of Mercury for many hours per day, allowing studies on morphology and variability, as connected to the Interplanetary Magnetic Field, as well as on Solar activity and intrinsic magnetosphere (e.g. Leblanc et al. 2008, 2009; Mangano et al. 2013, 2015; Massetti et al. 2017; Orsini et al. 2018; Milillo et al. 2020). In addition, THEMIS works perfectly also for elongations down to $10^{\circ}$ and it has recently installed an adaptive optics system that will greatly improve the spatial resolution of the Na exospheric maps produced. First Mercury observations are scheduled in early 2021. On the contrary, the McMath-Pierce Solar telescope has been now decommissioned (e.g. Potter and Killen 2008; Potter and Morgan 1990). It's role for future Mercury observations will be taken by the new generation solar telescope DKIST at Hawaii (the first call for proposals opened in summer 2020). DKIST, with the ViSP spectrograph, offers a promising follow-on: this next-generation telescope will initially have a $0.5^{\circ}-25^{\circ}$ exclusion zone in solar separation angles until safe operations with off-axis sunlight are characterized.

Observations of the $\mathrm{Na}$ tail are also of great interest: they show distinct, time variable north-south asymmetries within a few planetary radii and offer a several hour history of $\mathrm{Na}$ atmospheric escape rates at greater distances. These measurements are best performed 
Table 4 Earth-based observation parameters (elongation, illuminated disk fraction, phase angle) at the days of the Mercury flybys, and best suited observatories for daylight and twilight observations within 1 hour of CA. Suitable telescopes are: in Hawaii (Mauna Kea) Keck in evening twilight, CFHT in twilight; in Hawaii (Haleakala): DKIST in daylight and AEOS in twilight; in New Mexico: Dunn Solar Telescope in Daylight, Apache Point 3.5 in Twilight; in Arizona: Perkins in evening twilight, Discovery Channel Telescope in twilight; at the Canaries: THEMIS, GREGOR, and VTT telescopes in Daylight

\begin{tabular}{llllll}
\hline Flyby date & TAA & Elongation & Illuminated disk & Daylight obs. site & Twilight obs. site \\
\hline $2021-10-01$ & $260^{\circ}$ & $16^{\circ}$ & $12 \%$ & Hawaii & Hawaii \\
$2022-06-23$ & $263^{\circ}$ & $21^{\circ}$ & $52 \%$ & Canaries, New Mexico & Arizona \\
$2023-06-20$ & $312^{\circ}$ & $13^{\circ}$ & $86 \%$ & Hawaii & - \\
$2024-09-05$ & $331^{\circ}$ & $18^{\circ}$ & $47 \%$ & Canaries & - \\
$2024-12-02$ & $331^{\circ}$ & $9^{\circ}$ & $5 \%$ & Hawaii & Arizona \\
$2025-01-09$ & $150^{\circ}$ & $18^{\circ}$ & $86 \%$ & - & - \\
\hline
\end{tabular}

within short $\sim 30$ min viewing windows in twilight, which severely limits concurrent timings for coordinated mission support. $\mathrm{Na}$ tail observations can be made with traditional spectrographs within a few tens of planetary radii, or coronagraphic imaging at greater distances (e.g. Baumgardner et al. 2008; Schmidt et al. 2010). Observations of Mercury's potassium tail have been informally reported at conference proceedings and exhibit similar spatial structure to the $\sim 60$ times brighter $\mathrm{Na}$ tail. All other species in the exosphere tail require twilight observations at high spectral resolution. These must be measured off the bright planetary disk which scatters tens of MR/Å in solar continuum. The HIRES instrument on the Keck 1 telescope can observe calcium $4227 \AA$ emissions of a few kiloRayleighs (Bida et al. 2000; Killen et al. 2005). HIRES has also made tentative detections of iron and aluminum emissions of order tens of Rayleighs (Bida and Killen 2017). Comparison with MESSENGER UVVS data suggested that $\mathrm{Ca}^{+}$and Mn measurements may be within reach of HIRES as both at comparable brightness levels in the far blue (Vervack et al. 2016). All the more recently discovered faint species remain poorly characterized, being only studied so far as their initial detection.

Table 4 lists the dates of Mercury flybys together with the main Mercury parameters (as seen from the Earth), and potential optimal sites of Earth-based observations. Unfortunately during flybys, MCS configuration does not allow observations with SERENA neutral sensors (ELENA and STROFIO) perfectly suited for exospheric investigation; on the contrary, PHEBUS spectrometer will be operative. In addition, ion sensors will be able to operate (SERENA/MIPA and PICAM; SIXS, MPPE/MIA, and MPPE/MSA) and may give important information. Also MPPE/ENA may give additional important information, though partially shielded, of the energetic neutral atoms around Mercury, signature of high energy processes occurring in the interactions between the solar wind ions and the exospheric neutrals. Hence, Earth-based observations of the neutral components of $\mathrm{Na}$, for example, will be a fundamental complement to the measurements of in-situ ion component of the exosphere and magnetic field, for a first BepiColombo coordinated detection of the particle environment of Mercury.

According to Doppler shift observed at the Earth due to relative velocities of Mercury and the Earth, optimal observation of the $\mathrm{Na}$ exospheric emission could be performed in the Flybys 2 and 4. Flyby 2 (23 June 2022) is of particular interest. In fact, it is the one that will have wider coverage from the Earth, with daylight observations possible from the Canaries and New Mexico, and twilight ones from Arizona, with a 50\% of the disk illuminated, and highest elongation (and dawn terminator in view) (see Table 4). In addition, at 
Fig. 30 Mercury disk during Flyby 2 on 23 June 2022 as observed from BepiColombo (left) and from Earth (right) at three different times: 15:00 (top), 15:22 (center, time of CA) and 16:00 UTC (bottom). At the bottom of each planetary disk, first number is the apparent size of Mercury (in degrees or arcsecs) and $\alpha$ is the solar phase angle (in degrees)
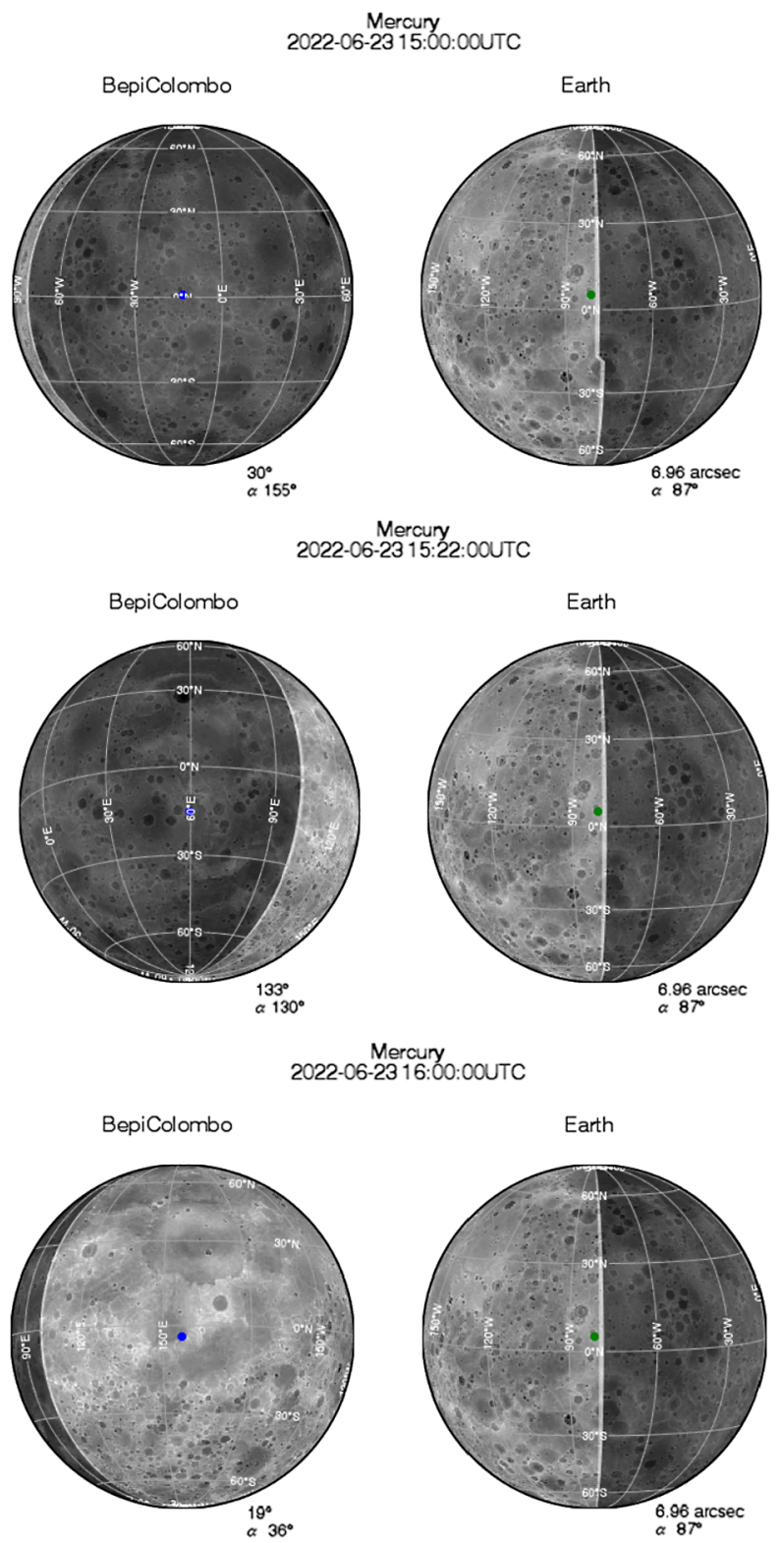

the Flyby 2 time, MCS is expected to approach Mercury from the night side and cross over the same portion of the surface viewed from the Earth shortly after. This means that, after in-situ measurements of the tail portion of the exosphere, direct comparison of global view images of the exosphere above the disk from the Earth with local in-situ measurements from PHEBUS can be performed. Figure 30 shows the illuminated portions of the disk and the expected longitudes as imaged by BepiColombo (left) and Earth-based telescopes (right) at three different times (before, during and after $\mathrm{CA}$ ). The occurrence of both daylight and twilight observing site will allow coverage of both the tail and the disk exosphere, along the whole flyby. 


\section{Summary}

BepiColombo, the ESA/JAXA cornerstone mission to Mercury launched on 20 October 2018 , is an ambitious project for the study of such a mysterious planet, still full of open questions (even after the recent MESSENGER mission). Though BepiColombo will begin its nominal mission in early 2026 with its two spacecraft finally orbiting in two different orbits around Mercury, it will be able to provide interesting and valuable measurements also during its long cruise (7.2 years) into the inner Solar System, even in its packed configuration that obstructs part of the scientific payload. The almost continuous measurements of MAG, ISA, MGNS and BERM onboard MPO from the launch up to now, as well as the overall data obtained during the recent flybys at the Earth and at Venus, clearly proved the scientific value as well as the feasibility of dedicated measurements also during cruise, and in planetary environments significantly different from the one for which the instrumentation has been designed.

Cruise science is divided into three main strands, related to the "deep space" cruise science (far from the planets), to the 2 Venus flybys, and to the 6 flybys of Mercury.

BepiColombo cruise science is very promising, thanks to the fact that a big part of the available instruments can provide good measurements also for interplanetary investigations.

In fact, BepiColombo is provided with two magnetometers, one of which (MPO-MAG) has the boom fully deployed and can work all the time allowed by ESA constraints on thrust arcs or other operations. In addition, electron and ion detectors (SERENA, SIXS, BERM, Mio/MPP), as well as neutron and gamma-ray spectrometers by MGNS (at different energy ranges) can be operative too. Finally, coordination with other spacecrafts and satellites presently operating in other regions of the inner Solar System may give additional vantage points of observations, and may provide multi-point analysis of different interplanetary medium features. In Table 5, potential science cases during the cruise phase are summarized.

Venus flyby investigations are the other key-point of the interplanetary cruise science, for Venus will be approached twice, on 15 October 2020, and on 10 August 2021, with two interesting flyby trajectories. In both cases, the evening terminator region will be approached at CA time, once by coming from the dayside and at high altitude (about $10700 \mathrm{~km}$ ), and a second time by arriving from the nightside, at a much lower altitude (down to about $550 \mathrm{~km}$ ). The two flyby configurations can allow valuable investigations of many different regions and phenomena of the interplanetary-planetary induced magnetosphere, plus of the Venus' atmospheric composition and thermal profiles. In the following, Tables 6, 7, and 8 show a summary of the potential science cases to be investigated by the BepiColombo instrumentation during the flybys at Venus (divided by scientific goal: atmosphere, magnetosphere and mixed/of support to the previous two).

Finally, Mercury itself will be the last (and primary) focus of the interplanetary investigations of BepiColombo. The main target of the BepiColombo mission will be approached for the first time already in October 2021, when the first of six flybys will occur to allow progressive modifications to the interplanetary cruise trajectory to reach the final orbit around Mercury in December 2025. Hence, the six flybys will allow the first in-situ measurements of the different instruments of BepiColombo four years in advance before its orbit insertion. In addition, the different geometry of the Mercury flybys trajectories (and the low altitude of CA of many of them down to $200 \mathrm{~km}$ ) will allow investigations of regions that have never been investigated by previous missions, and first BepiColombo measurements of magnetosphere, exosphere, surface and interior will be addressed. 


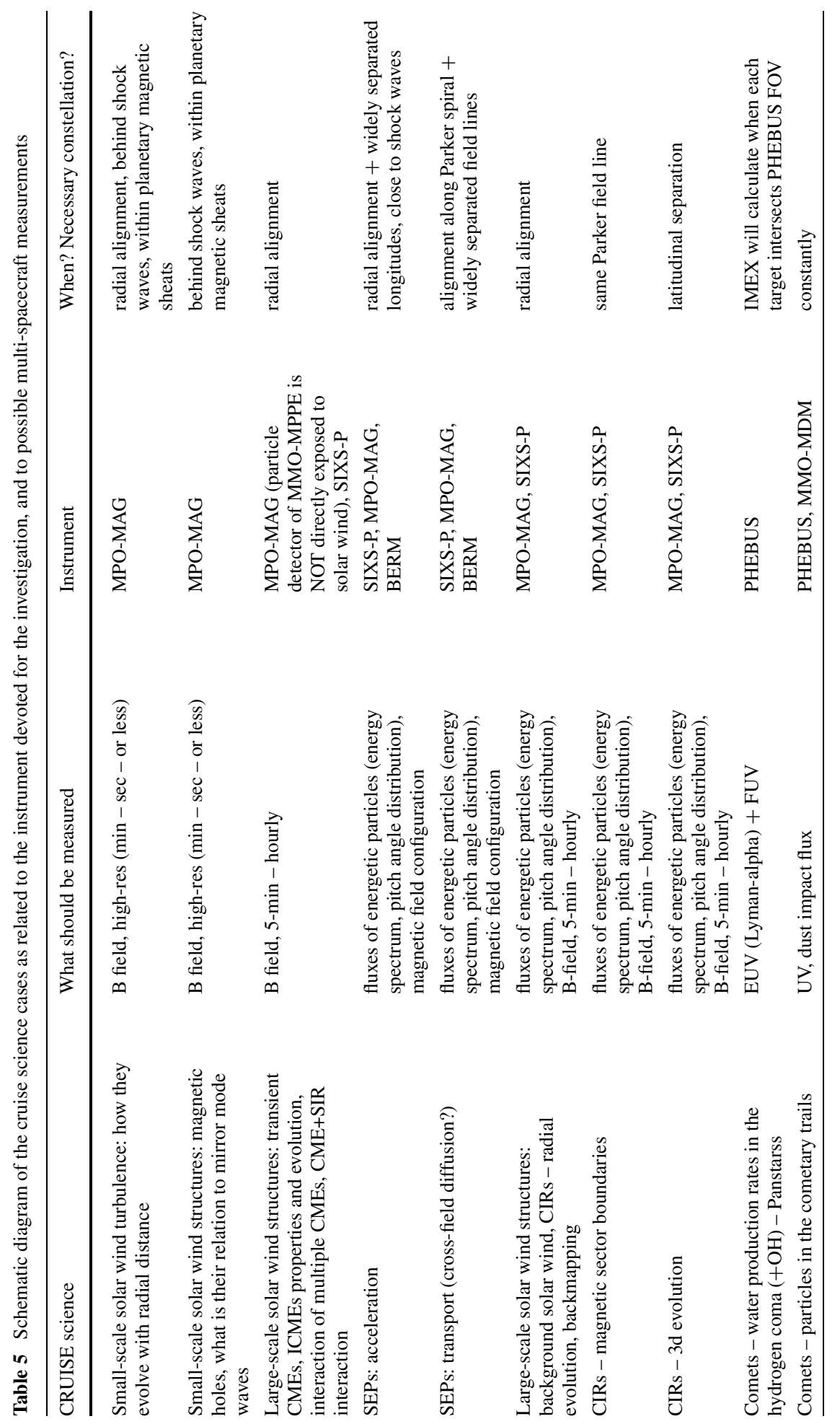




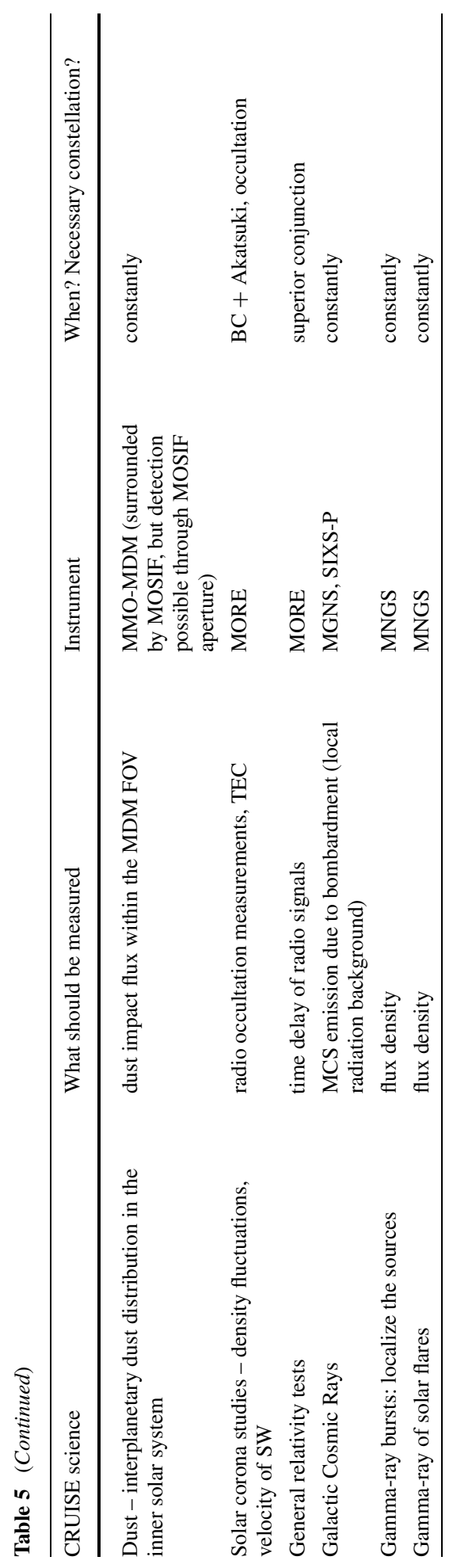




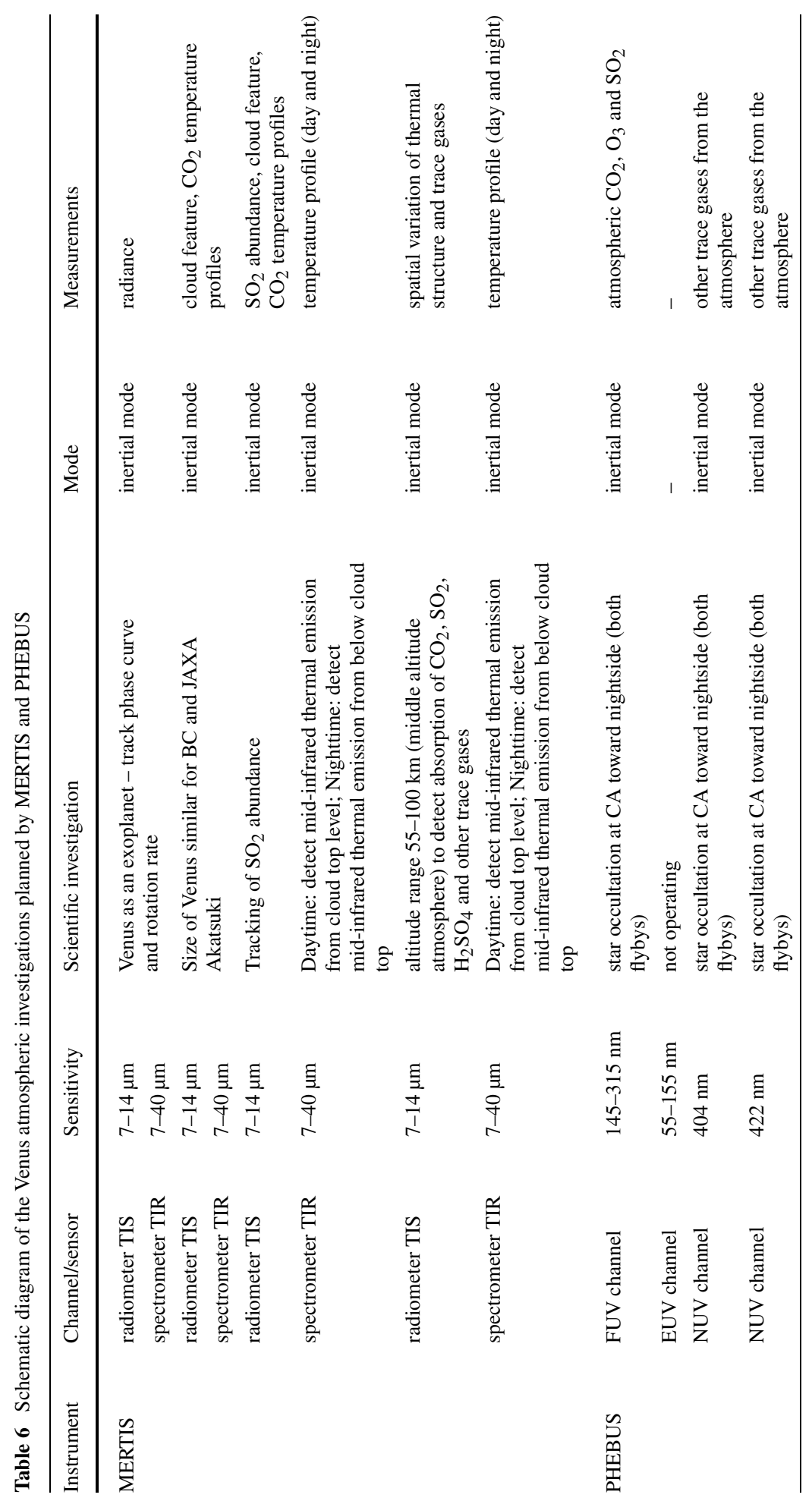




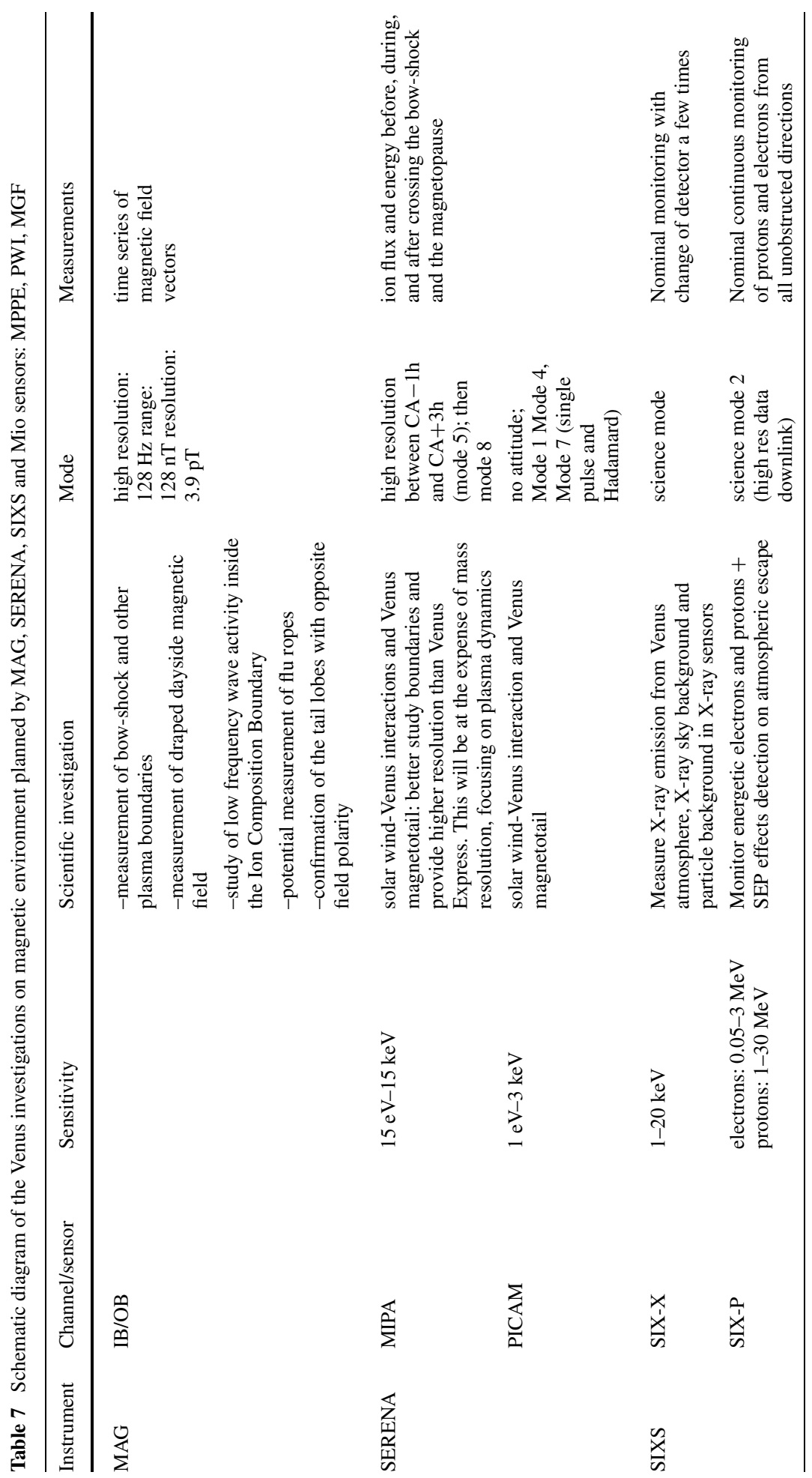




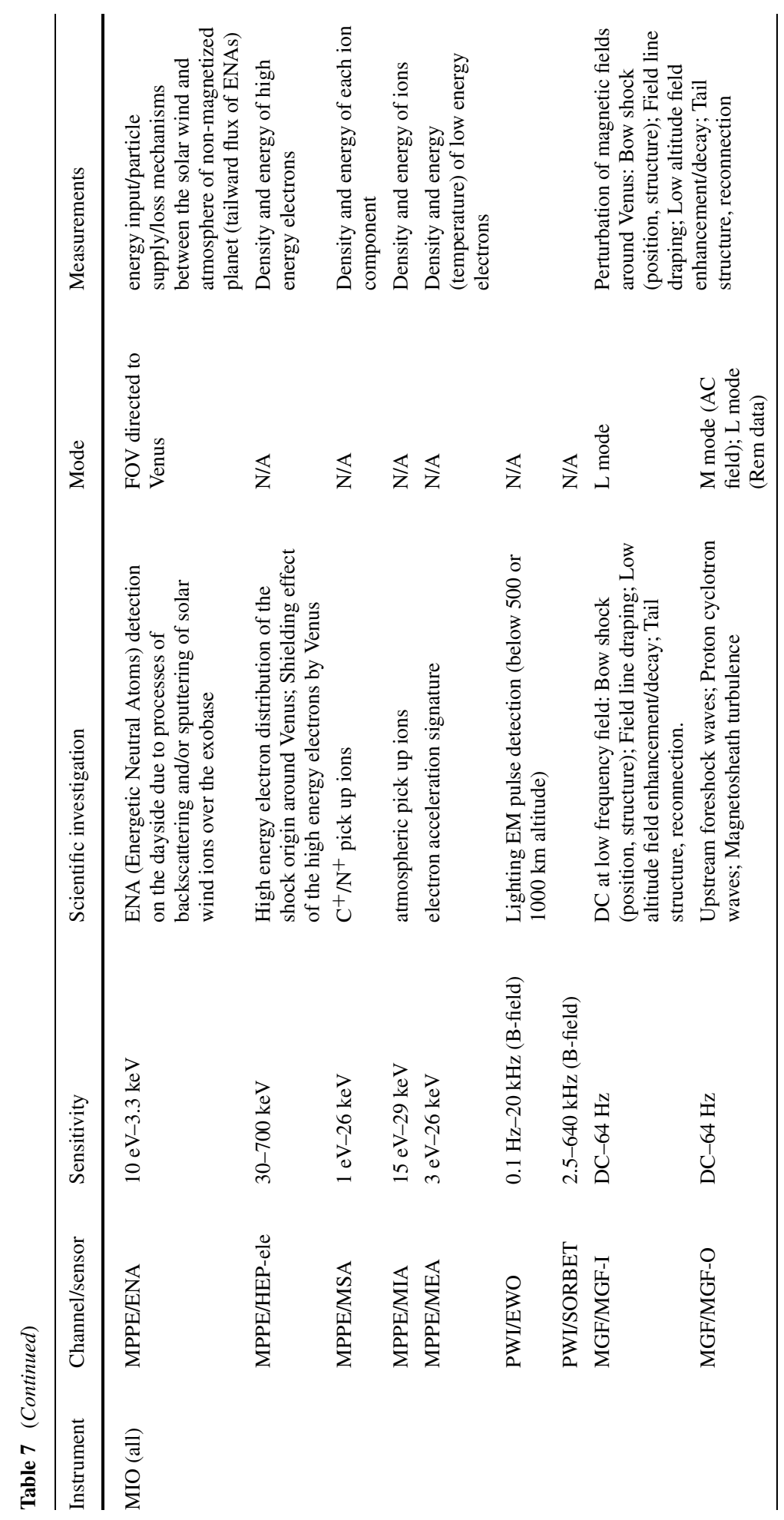




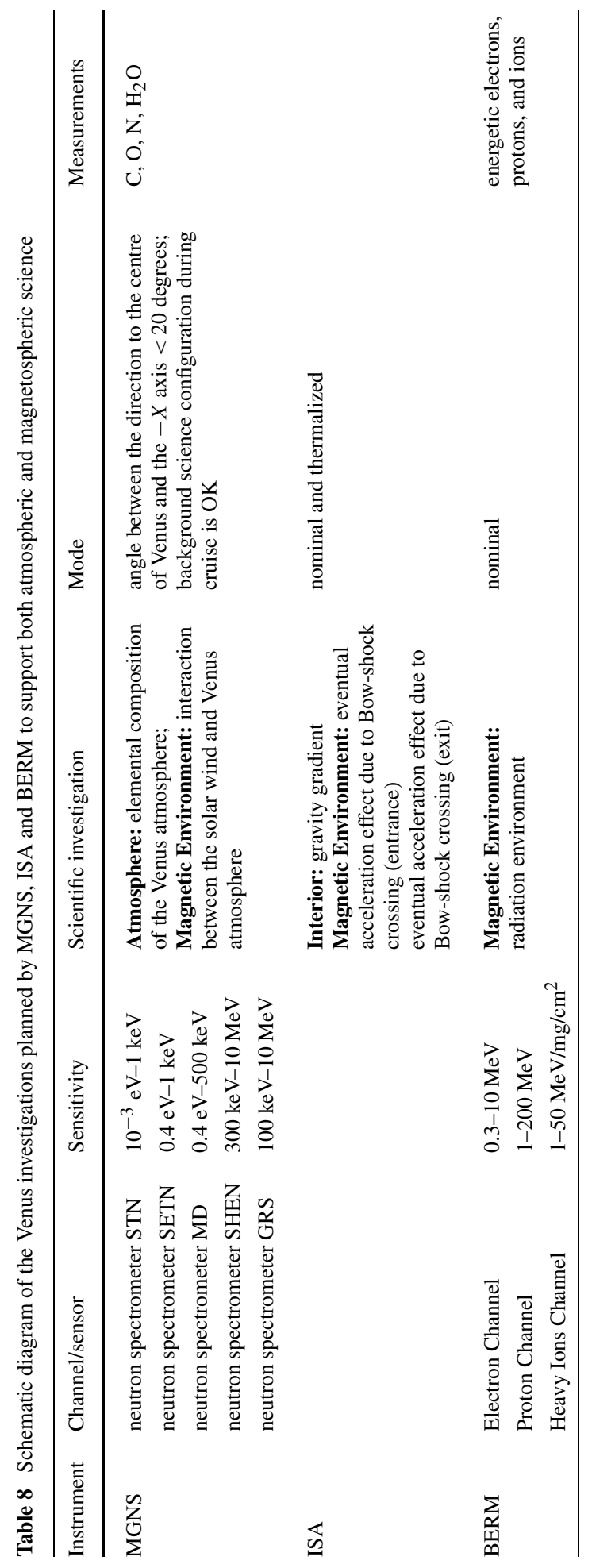




\section{Conclusions}

In conclusion, in the present paper we collected a wide range of possible science cases that the BepiColombo mission will approach in the present years during its cruise phase towards Mercury. Science cases include mainly four different environments: the Earth, the planet Venus and planet Mercury during the flybys, and the interplanetary environment during cruise (far from planets). The science goals will be addressed by mean of strong synergy among the onboard instrumentation, but also in coordination with other spacecraft presently operating in the inner Solar System, and with Earth-based telescopes observations.

Acknowledgements The authors would like to thank the two anonymous referees who kindly reviewed the first version of this manuscript and provided valuable suggestions and comments to improve its quality.

A special thanks to Prof. Dario Del Moro (University of Rome Tor Vergata) for fruitful suggestions and support on space weather modeling section, and on editing.

In addition:

A. Madár and M. Dósa were funded by the National Research, Development and Innovation Office NKFIH, grant FK128548.

Y.J. Lee has received funding from EU Horizon 2020 MSCA-IF No. 841432.

J.S. Oliveira is funded by the ESA Research Fellowship program in Space Science.

C. Dong was supported by NASA under Proposal Grants 80NSSC19K0161 and 80NSSC20K1416.

C. Schmidt acknowledges support from NASA programs 80NSSC19K0790, 80NSSC18K0857 and 80NSSC21K0051.

The MGNS instrument team in Russia is funded by Ministry of Science and Higher Education of the Russian Federation, grant AAAA-A18-118012290370-6.

The IMEX Dust Streams in Space model was developed under ESA funding (contract 4000106316/12/ NL/AF-IMEX).

Funding Note Open Access funding provided by Istituto Nazionale di Astrofisica within the CRUI-CARE Agreement.

Publisher's Note Springer Nature remains neutral with regard to jurisdictional claims in published maps and institutional affiliations.

Open Access This article is licensed under a Creative Commons Attribution 4.0 International License, which permits use, sharing, adaptation, distribution and reproduction in any medium or format, as long as you give appropriate credit to the original author(s) and the source, provide a link to the Creative Commons licence, and indicate if changes were made. The images or other third party material in this article are included in the article's Creative Commons licence, unless indicated otherwise in a credit line to the material. If material is not included in the article's Creative Commons licence and your intended use is not permitted by statutory regulation or exceeds the permitted use, you will need to obtain permission directly from the copyright holder. To view a copy of this licence, visit http://creativecommons.org/licenses/by/4.0/.

\section{Appendix}

Table 9 Sun offset allowed range in $Y$ direction during cruise. SAA is the Sun Avoidance Angle; SEP here is for Solar Electric Propulsion system; (*) when not operational

\begin{tabular}{lll}
\hline SAA details & SAA & Constraints \\
\hline minimum value & $-9.1^{\circ}$ & MOSIF cut-off plane at $-18^{\circ}$ \\
maximum at $0.8 \mathrm{AU}$ & $+47^{\circ}$ & SEP illumination up to $50^{\circ}\left(^{*}\right)$ \\
maximum between 0.7 & $+27^{\circ}$ & SEP illumination up to $30^{\circ}(*)$ \\
and $0.8 \mathrm{AU}$ & & SEP illumination up to $17^{\circ}(*)$ \\
maximum at $<0.7 \mathrm{AU}$ & $+14^{\circ}$ & S
\end{tabular}


Table 10 List of programmed solar Electric Propulsion operational periods along BepiColombo cruise. From BC-ESC-RP-0550_BC_CReMA document, Issue 5.4, June 2018

\begin{tabular}{|c|c|c|c|}
\hline Nr. & Duration (days) & Start time & End time \\
\hline EP1 & 66 & $16-12-2018$ & 19-02-2019 \\
\hline EP2 & 60 & 22-09-2019 & 20-11-2019 \\
\hline EP3 & 5 & $12-02-2020$ & $16-02-2020$ \\
\hline EP4 & 17 & $18-06-2021$ & 04-07-2021 \\
\hline EP5 & 8 & $18-08-2021$ & $25-08-2021$ \\
\hline EP6 & 5 & 09-10-2021 & $13-10-2021$ \\
\hline EP7 & 6 & 06-12-2021 & $11-12-2021$ \\
\hline EP8 & 22 & 05-02-2022 & 26-02-2022 \\
\hline EP9 & 6 & $30-06-2022$ & $05-07-2022$ \\
\hline EP10 & 5 & $21-08-2022$ & $25-08-2022$ \\
\hline EP11 & 34 & 01-12-2022 & 03-01-2023 \\
\hline EP12 & 46 & $23-03-2023$ & $07-05-2023$ \\
\hline EP13 & 34 & $12-08-2023$ & 14-09-2023 \\
\hline EP14 & 16 & $18-12-2023$ & $02-01-2024$ \\
\hline EP15 & 29 & 21-01-2024 & $18-02-2024$ \\
\hline EP16 & 48 & $25-04-2024$ & $11-06-2024$ \\
\hline EP17 & 12 & $12-09-2024$ & 23-09-2024 \\
\hline EP18 & 11 & $14-10-2024$ & 24-10-2024 \\
\hline EP19 & 29 & 19-01-2025 & $16-02-2025$ \\
\hline EP20 & 35 & 26-02-2025 & 01-04-2025 \\
\hline EP21 & 70 & 24-04-2025 & $02-07-2025$ \\
\hline EP22 & 44 & $15-08-2025$ & 27-09-2025 \\
\hline
\end{tabular}

\section{References}

V. Airapetian, R. Barnes, O. Cohen, G. Collinson, W. Danchi, C. Dong, A. Del Genio, K. France, K. GarciaSage, A. Glocer et al., Impact of space weather on climate and habitability of terrestrial-type exoplanets. Int. J. Astrobiol. 19(2), 136-194 (2020)

T. Alberti, M. Laurenza, G. Consolini, A. Milillo, M.F. Marcucci, V. Carbone, S.D. Bale, On the scaling properties of magnetic field fluctuations through the inner heliosphere. ArXiv preprint (2020). arXiv:2006.03285

N. Altobelli, E. Grün, M. Landgraf, A new look into the Helios dust experiment data: presence of interstellar dust inside the Earth's orbit. Astron. Astrophys. 448(1), 243-252 (2006)

B.J. Anderson, M.H. Acuña, D.A. Lohr, J. Scheifele, A. Raval, H. Korth, J.A. Slavin, The Magnetometer Instrument on MESSENGER. Space Sci. Rev. 131(1-4), 417-450 (2007). https://doi.org/10.1007/ s11214-007-9246-7

B.J. Anderson, C.L. Johnson, H. Korth, M.E. Purucker, R.M. Winslow, J.A. Slavin, S.C. Solomon, R.L. McNutt, J.M. Raines, T.H. Zurbuchen, The global magnetic field of Mercury from MESSENGER orbital observations. Science 333(6051), 1859 (2011). https://doi.org/10.1126/science.1211001

B.J. Anderson, C.L. Johnson, H. Korth, R.M. Winslow, J.E. Borovsky, M.E. Purucker, J.A. Slavin, S.C. Solomon, M.T. Zuber, J. McNutt, L. Ralph, Low-degree structure in Mercury's planetary magnetic field. J. Geophys. Res., Planets 117, E00L12 (2012). https://doi.org/10.1029/2012JE004159

B.J. Anderson, C.L. Johnson, H. Korth, J.A. Slavin, R.M. Winslow, R.J. Phillips, R.L. McNutt, S.C. Solomon, Steady-state field-aligned currents at Mercury. Geophys. Res. Lett. 41(21), 7444-7452 (2014)

B. Andreichikov, Chemical composition and structure of Venus clouds from results of X-ray radiometric experiments made with the Vega 1 and Vega 2 automatic interplanetary stations. Kosm. Issled. 25, 737743 (1987)

C.N. Arge, V.J. Pizzo, Improvement in the prediction of solar wind conditions using near-real time solar magnetic field updates. J. Geophys. Res. 105(A5), 10465-10480 (2000). https://doi.org/10.1029/ 1999JA000262 
A. Bader, G. Stenberg Wieser, M. André, M. Wieser, Y. Futaana, M. Persson, H. Nilsson, T.L. Zhang, Proton temperature anisotropies in the plasma environment of Venus. J. Geophys. Res. Space Phys. 124(5), 3312-3330 (2019). https://doi.org/10.1029/2019JA026619

S. Barabash, A. Fedorov, J.J. Sauvaud, R. Lundin, C.T. Russell, Y. Futaana, T.L. Zhang, H. Andersson, K. Brinkfeldt, A. Grigoriev, M. Holmström, M. Yamauchi, K. Asamura, W. Baumjohann, H. Lammer, A.J. Coates, D.O. Kataria, D.R. Linder, C.C. Curtis, K.C. Hsieh, B.R. Sandel, M. Grande, H. Gunell, H.E.J. Koskinen, E. Kallio, P. Riihelä, T. Säles, W. Schmidt, J. Kozyra, N. Krupp, M. Fränz, J. Woch, J. Luhmann, S. McKenna-Lawlor, C. Mazelle, J.J. Thocaven, S. Orsini, R. Cerulli-Irelli, M. Mura, M. Milillo, M. Maggi, E. Roelof, P. Brandt, K. Szego, J.D. Winningham, R.A. Frahm, J. Scherrer, J.R. Sharber, P. Wurz, P. Bochsler, The loss of ions from Venus through the plasma wake. Nature 450(7170), 650-653 (2007a). https://doi.org/10.1038/nature06434

S. Barabash, J.A. Sauvaud, H. Gunell, H. Andersson, A. Grigoriev, K. Brinkfeldt, M. Holmström, R. Lundin, M. Yamauchi, K. Asamura, W. Baumjohann, T.L. Zhang, A.J. Coates, D.R. Linder, D.O. Kataria, C.C. Curtis, K.C. Hsieh, B.R. Sandel, A. Fedorov, C. Mazelle, J.J. Thocaven, M. Grande, H.E.J. Koskinen, E. Kallio, T. Säles, P. Riihela, J. Kozyra, N. Krupp, J. Woch, J. Luhmann, S. McKenna-Lawlor, S. Orsini, R. Cerulli-Irelli, M. Mura, M. Milillo, M. Maggi, E. Roelof, P. Brandt, C.T. Russell, K. Szego, J.D. Winningham, R.A. Frahm, J. Scherrer, J.R. Sharber, P. Wurz, P. Bochsler, The Analyser of Space Plasmas and Energetic Atoms (ASPERA-4) for the Venus Express mission. Planet. Space Sci. 55(12), 1772-1792 (2007b). https://doi.org/10.1016/j.pss.2007.01.014

J. Baumgardner, J. Wilson, M. Mendillo, Imaging the sources and full extent of the sodium tail of the planet Mercury. Geophys. Res. Lett. 35(3), L03201 (2008). https://doi.org/10.1029/2007GL032337

W. Baumjohann, A. Matsuoka, Y. Narita, W. Magnes, D. Heyner, K.H. Glassmeier, R. Nakamura, D. Fischer, F. Plaschke, M. Volwerk et al., The BepiColombo-Mio magnetometer en route to Mercury. Space Sci. Rev. 216(8), 1-33 (2020)

D.A. Belyaev, D.G. Evdokimova, F. Montmessin, J.L. Bertaux, O.I. Korablev, A.A. Fedorova, E. Marcq, L. Soret, M.S. Luginin, Night side distribution of $\mathrm{SO}_{2}$ content in Venus' upper mesosphere. Icarus 294, 58-71 (2017). https://doi.org/10.1016/j.icarus.2017.05.002

J.L. Bertaux, J.E. Blamont, V.M. Lepine, V.G. Kurt, N.N. Romanova, A.S. Smirnov, Venera 11 and Venera 12 observations of e.u.v. emissions from the upper atmosphere of Venus. Planet. Space Sci. 29(2), 149-166 (1981). https://doi.org/10.1016/0032-0633(81)90029-5

J.L. Bertaux, E. Kyrölä, E. Quémerais, R. Pellinen, R. Lallement, W. Schmidt, M. Berthe, E. Dimarellis, J.P. Goutail, C. Taulemesse et al., Swan: a study of Solar Wind Anisotropies on SOHO with Lyman alpha sky mapping, in The SOHO Mission (Springer, Berlin, 1995), pp. 403-439

J.L. Bertaux, D. Nevejans, O. Korablev, E. Villard, E. Quémerais, E. Neefs, F. Montmessin, F. Leblanc, J.P. Dubois, E. Dimarellis, A. Hauchecorne, F. Lefèvre, P. Rannou, J.Y. Chaufray, M. Cabane, G. Cernogora, G. Souchon, F. Semelin, A. Reberac, E. Van Ransbeek, S. Berkenbosch, R. Clairquin, C. Muller, F. Forget, F. Hourdin, O. Talagrand, A. Rodin, A. Fedorova, A. Stepanov, I. Vinogradov, A. Kiselev, Y. Kalinnikov, G. Durry, B. el Sand, A. Stern, J.C. Gérard, SPICAV on Venus Express: three spectrometers to study the global structure and composition of the Venus atmosphere. Planet. Space Sci. 55(12), 16731700 (2007). https://doi.org/10.1016/j.pss.2007.01.016

B. Bertotti, G. Comoretto, L. Iess, Doppler tracking of spacecraft with multi-frequency links. Astron. Astrophys. 269, 608-616 (1993)

B. Bertotti, L. Iess, P. Tortora, A test of general relativity using radio links with the Cassini spacecraft. Nature 425(6956), 374-376 (2003)

T.A. Bida, R.M. Killen, Observations of the minor species Al and Fe in Mercury's exosphere. Icarus 289, 227-238 (2017). https://doi.org/10.1016/j.icarus.2016.10.019

T.A. Bida, R.M. Killen, T.H. Morgan, Discovery of calcium in Mercury's atmosphere. Nature 404(6774), 159-161 (2000). https://doi.org/10.1038/35004521

D. Bohm, E.P. Gross, Theory of plasma oscillations. B. Excitation and damping of oscillations. Phys. Rev. 75(12), 1864 (1949)

V. Bothmer, R. Schwenn, The structure and origin of magnetic clouds in the solar wind. Ann. Geophys. 16(1), 1-24 (1998). https://doi.org/10.1007/s00585-997-0001-x

L.H. Brace, R.F. Theis, H.G. Mayr, S.A. Curtis, J.G. Luhmann, Holes in the nightside ionosphere of Venus. J. Geophys. Res. 87(A1), 199-211 (1982). https://doi.org/10.1029/JA087iA01p00199

D.A. Brain, J. McFadden, J.S. Halekas, J. Connerney, S.W. Bougher, S. Curry, C. Dong, Y. Dong, F. Eparvier, $X$. Fang et al., The spatial distribution of planetary ion fluxes near Mars observed by MAVEN. Geophys. Res. Lett. 42(21), 9142-9148 (2015)

R. Bruno, V. Carbone, Turbulence in the Solar Wind (Springer, Berlin, 2016)

R. Bruno, V. Carbone, Z. Vörös, R. D’Amicis, B. Bavassano, M.B. Cattaneo, A. Mura, A. Milillo, S. Orsini, P. Veltri, L. Sorriso-Valvo, T. Zhang, H. Biernat, H. Rucker, W. Baumjohann, D. Jankovičová, P. Kovács, Coordinated study on solar wind turbulence during the Venus-Express, ACE and Ulysses 
alignment of August 2007. Earth Moon Planets 104(1-4), 101-104 (2009). https://doi.org/10.1007/ s11038-008-9272-9

L.F. Burlaga, Magnetic clouds and force-free fields with constant alpha. J. Geophys. Res. 93(A7), 7217-7224 (1988). https://doi.org/10.1029/JA093iA07p07217

L.F. Burlaga, K.W. Ogilvie, D.H. Fairfield, Microscale fluctuations in the interplanetary magnetic field. Astrophys. J. Lett. 155, L171 (1969). https://doi.org/10.1086/180329

H.V. Cane, I.G. Richardson, Interplanetary coronal mass ejections in the near-Earth solar wind during 19962002. J. Geophys. Res. Space Phys. 108(A4), 1156 (2003). https://doi.org/10.1029/2002JA009817

A. Cardesín Moinelo, G. Piccioni, A. Migliorini, P. Drossart, Global mapping of Venus' atmosphere using accumulated projections of VIRTIS Venus Express observations, in AGUFM 2008:P22A-04 (2008)

T.A. Cassidy, A.W. Merkel, M.H. Burger, M. Sarantos, R.M. Killen, W.E. McClintock, R.J. Vervack Jr., Mercury's seasonal sodium exosphere: MESSENGER orbital observations. Icarus 248, 547-559 (2015)

J.Y. Chaufray, J.L. Bertaux, UV cometary observations by SPICAV on Venus Express, in European Planetary Science Congress, EPSC2015-88 (2015)

J.Y. Chaufray, J.L. Bertaux, E. Quémerais, E. Villard, F. Leblanc, Hydrogen density in the dayside Venusian exosphere derived from Lyman- $\alpha$ observations by SPICAV on Venus Express. Icarus 217(2), 767-778 (2012). https://doi.org/10.1016/j.icarus.2011.09.027

C. Chen, S. Bale, J. Bonnell, D. Borovikov, T. Bowen, D. Burgess, A. Case, B. Chandran, T.D. de Wit, K. Goetz et al., The evolution and role of solar wind turbulence in the inner heliosphere. Astrophys. J. Suppl. Ser. 246(2), 53 (2020)

R.N. Clark, Detection of adsorbed water and hydroxyl on the Moon. Science 326(5952), 562 (2009). https:// doi.org/10.1126/science. 1178105

M.R. Combi, T. Mäkinen, J.L. Bertaux, E. Quémerais, S. Ferron, M. Avery, C. Wright, Water production activity of nine long-period comets from SOHO/SWAN observations of hydrogen Lyman-alpha: 20132016. Icarus 300, 33-46 (2018)

V. Cottini, N.I. Ignatiev, G. Piccioni, P. Drossart, Water vapor near Venus cloud tops from VIRTIS-H/Venus Express observations 2006-2011. Planet. Space Sci. 113, 219-225 (2015). https://doi.org/10.1016/ j.pss.2015.03.012

T.E. Cravens, L.H. Brace, H.A. Taylor, C.T. Russell, W.L. Knudsen, K.L. Miller, A. Barnes, J.D. Mihalov, F.L. Scarf, S.J. Quenon, A.F. Nagy, Disappearing ionospheres on the nightside of Venus. Icarus 51(2), 271-282 (1982). https://doi.org/10.1016/0019-1035(82)90083-5

J.L. Culhane, L.K. Harra, A.M. James, K. Al-Janabi, L.J. Bradley, R.A. Chaudry, K. Rees, J.A. Tandy, P. Thomas, M.C.R. Whillock, B. Winter, G.A. Doschek, C.M. Korendyke, C.M. Brown, S. Myers, J. Mariska, J. Seely, J. Lang, B.J. Kent, B.M. Shaughnessy, P.R. Young, G.M. Simnett, C.M. Castelli, S. Mahmoud, H. Mapson-Menard, B.J. Probyn, R.J. Thomas, J. Davila, K. Dere, D. Windt, J. Shea, R. Hagood, R. Moye, H. Hara, T. Watanabe, K. Matsuzaki, T. Kosugi, V. Hansteen, O. Wikstol, The EUV imaging spectrometer for Hinode. Sol. Phys. 243(1), 19-61 (2007). https://doi.org/10.1007/ s01007-007-0293-1

S. Curry, M. Liemohn, X. Fang, Y. Ma, J. Slavin, J. Espley, S. Bougher, C. Dong, Test particle comparison of heavy atomic and molecular ion distributions at Mars. J. Geophys. Res. Space Phys. 119(3), 2328-2344 (2014)

S. Dasso, M.S. Nakwacki, P. Démoulin, C.H. Mandrini, Progressive transformation of a flux rope to an ICME. Comparative analysis using the direct and fitted expansion methods. Sol. Phys. 244(1-2), 115137 (2007). https://doi.org/10.1007/s11207-007-9034-2. 0706.2889

M. Delva, C. Bertucci, M. Volwerk, R. Lundin, C. Mazelle, N. Romanelli, Upstream proton cyclotron waves at Venus near solar maximum. J. Geophys. Res. Space Phys. 120(1), 344-354 (2015). https://doi.org/ 10.1002/2014JA020318

K. Dennerl, Charge transfer reactions. Space Sci. Rev. 157(1-4), 57-91 (2010). https://doi.org/10.1007/ s11214-010-9720-5

M. Desai, J. Giacalone, Large gradual solar energetic particle events. Living Rev. Sol. Phys. 13(1), 3 (2016). https://doi.org/10.1007/s41116-016-0002-5

G.A. DiBraccio, J.A. Slavin, S.M. Imber, D.J. Gershman, J.M. Raines, C.M. Jackman, S.A. Boardsen, B.J. Anderson, H. Korth, T.H. Zurbuchen, R.L. McNutt, S.C. Solomon, MESSENGER observations of flux ropes in Mercury's magnetotail. Planet. Space Sci. 115, 77-89 (2015). https://doi.org/10.1016/ j.pss.2014.12.016

C. Dong, M. Jin, M. Lingam, V.S. Airapetian, Y. Ma, B. van der Holst, Atmospheric escape from the TRAPPIST-1 planets and implications for habitability. Proc. Natl. Acad. Sci. 115(2), 260-265 (2018a)

C. Dong, L. Wang, Y.M. Huang, L. Comisso, A. Bhattacharjee, Role of the plasmoid instability in magnetohydrodynamic turbulence. Phys. Rev. Lett. 121(16), 165101 (2018b) 
C. Dong, L. Wang, A. Hakim, A. Bhattacharjee, J.A. Slavin, G.A. DiBraccio, K. Germaschewski, Global ten-moment multifluid simulations of the solar wind interaction with Mercury: from the planetary conducting core to the dynamic magnetosphere. Geophys. Res. Lett. 46(21), 11584-11596 (2019)

C. Dong, M. Jin, M. Lingam, Atmospheric escape from TOI-700 d: Venus versus Earth analogs. Astrophys. J. Lett. 896(2), L24 (2020)

M. Dósa, G. Erdős, Long-term longitudinal recurrences of the open magnetic flux density in the heliosphere. Astrophys. J. 838(2), 104 (2017)

N. Dresing, R. Gómez-Herrero, B. Heber, A. Klassen, O. Malandraki, W. Dröge, Y. Kartavykh, Statistical survey of widely spread out solar electron events observed with STEREO and ACE with special attention to anisotropies. Astron. Astrophys. 567, A27 (2014). https://doi.org/10.1051/0004-6361/201423789

M.R. Drinkwater, R. Haagmans, D. Muzi, A. Popescu, R. Floberghagen, M. Kern, M. Fehringer, The GOCE gravity mission: ESA's first core Earth explorer, in Proceedings of the 3rd International GOCE User Workshop. Citeseer (2006), pp. 6-8

E. Dubinin, M. Fraenz, A. Fedorov, R. Lundin, N. Edberg, F. Duru, O. Vaisberg, Ion energization and escape on Mars and Venus. Space Sci. Rev. 162(1-4), 173-211 (2011). https://doi.org/10.1007/ s11214-011-9831-7

E. Dubinin, M. Fraenz, T.L. Zhang, J. Woch, Y. Wei, A. Fedorov, S. Barabash, R. Lundin, Plasma in the near Venus tail: Venus Express observations. J. Geophys. Res. Space Phys. 118(12), 7624-7634 (2013). https://doi.org/10.1002/2013JA019164

M. Dumbović, J. Ćalogović, B. Vršnak, M. Temmer, M.L. Mays, A. Veronig, I. Piantschitsch, The DragBased Ensemble Model (DBEM) for coronal mass ejection propagation. Astrophys. J. 854(2), 180 (2018)

R.C. Elphic, C.T. Russell, J.G. Luhmann, F.L. Scarf, L.H. Brace, The Venus ionopause current sheet: thickness length scale and controlling factors. J. Geophys. Res. 86(A13), 11430-11438 (1981). https:// doi.org/10.1029/JA086iA13p11430

T. Encrenaz, T.K. Greathouse, E. Marcq, H. Sagawa, T. Widemann, B. Bézard, T. Fouchet, F. Lefèvre, S. Lebonnois, S.K. Atreya, Y.J. Lee, R. Giles, S. Watanabe, HDO and $\mathrm{SO}_{2}$ thermal mapping on Venus. IV. Statistical analysis of the $\mathrm{SO}_{2}$ plumes. Astron. Astrophys. 623, A70 (2019). https://doi.org/ 10.1051/0004-6361/201833511

L.G. Evans, P.N. Peplowski, E.A. Rhodes, J.O. Goldsten, R.D. Starr, S.C. Solomon, The MESSENGER Gamma-Ray Spectrometer: calibration and operations. Icarus 288, 186-200 (2017)

W. Exner, D. Heyner, L. Liuzzo, U. Motschmann, D. Shiota, K. Kusano, T. Shibayama, Coronal mass ejection hits Mercury: A.I.K.E.F. hybrid-code results compared to MESSENGER data. Planet. Space Sci. 153, 89-99 (2018)

C.J. Eyles, R.A. Harrison, C.J. Davis, N.R. Waltham, B.M. Shaughnessy, H.C.A. Mapson-Menard, D. Bewsher, S.R. Crothers, J.A. Davies, G.M. Simnett, R.A. Howard, J.D. Moses, J.S. Newmark, D.G. Socker, J.P. Halain, J.M. Defise, E. Mazy, P. Rochus, The heliospheric imagers onboard the STEREO mission. Sol. Phys. 254(2), 387-445 (2009). https://doi.org/10.1007/s11207-008-9299-0

A. Fedorov, S. Barabash, J.A. Sauvaud, Y. Futaana, T.L. Zhang, R. Lundin, C. Ferrier, Measurements of the ion escape rates from Venus for solar minimum. J. Geophys. Res. Space Phys. 116(A7), A07220 (2011). https://doi.org/10.1029/2011JA016427

G. Fjeldbo, V.R. Eshleman, Atmosphere of Venus as studied with the Mariner 5 dual radio-frequency occultation experiment. Radio Sci. 4(10), 879-897 (1969). https://doi.org/10.1029/RS004i010p00879

J.L. Fox, Morphology of the dayside ionosphere of Venus: implications for ion outflows. J. Geophys. Res., Planets 113(E11), E11001 (2008). https://doi.org/10.1029/2008JE003182

J.L. Fox, W.T. Kasprzak, Near-terminator Venus ionosphere: evidence for a dawn/dusk asymmetry in the thermosphere. J. Geophys. Res., Planets 112(E9), E09008 (2007). https://doi.org/10.1029/2007JE002899

M. Fränz, E. Echer, A. Marques de Souza, E. Dubinin, T.L. Zhang, Ultra low frequency waves at Venus: observations by the Venus Express spacecraft. Planet. Space Sci. 146, 55-65 (2017). https://doi.org/ 10.1016/j.pss.2017.08.011

K. Fujiki, M. Kojima, M. Tokumaru, T. Ohmi, A. Yokobe, K. Hayashi, D.J. McComas, H.A. Elliott, How did the solar wind structure change around the solar maximum? From interplanetary scintillation observation. Ann. Geophys. 21(6), 1257-1261 (2003). https://doi.org/10.5194/angeo-21-1257-2003

T. Fukuhara, M. Futaguchi, G.L. Hashimoto, T. Horinouchi, T. Imamura, N. Iwagaimi, T. Kouyama, S.Y. Murakami, M. Nakamura, K. Ogohara, M. Sato, T.M. Sato, M. Suzuki, M. Taguchi, S. Takagi, M. Ueno, S. Watanabe, M. Yamada, A. Yamazaki, Large stationary gravity wave in the atmosphere of Venus. Nat. Geosci. 10(2), 85-88 (2017). https://doi.org/10.1038/ngeo2873

Y. Futaana, G. Stenberg Wieser, S. Barabash, J.G. Luhmann, Solar wind interaction and impact on the Venus atmosphere. Space Sci. Rev. 212(3-4), 1453-1509 (2017). https://doi.org/10.1007/s11214-017-0362-8

I. Garate-Lopez, R. Hueso, Y.J. Lee, V. Mangano, K.L. Jessup, J. Peralta, A. Sanchez-Lavega, J. Zender, J. Benkhoff, G. Murakami, M. Scherf, Amateur ground-based support of the first BepiColombo 
flyby of Venus, in Europlanet Science Congress 2020, Online, vol. 14 (2020). https://doi.org/10.5194/ epsc2020-1060

J.C. Gérard, J. Gustin, B. Hubert, G.R. Gladstone, L.W. Esposito, Measurements of the helium $584 \AA$ airglow during the Cassini flyby of Venus. Planet. Space Sci. 59(13), 1524-1528 (2011a). https:// doi.org/10.1016/j.pss.2011.06.018

J.C. Gérard, B. Hubert, J. Gustin, V.I. Shematovich, D. Bisikalo, G.R. Gladstone, L.W. Esposito, EUV spectroscopy of the Venus dayglow with UVIS on Cassini. Icarus 211(1), 70-80 (2011b). https:// doi.org/10.1016/j.icarus.2010.09.020

D.J. Gershman, J.A. Slavin, J.M. Raines, T.H. Zurbuchen, B.J. Anderson, H. Korth, D.N. Baker, S.C. Solomon, Magnetic flux pileup and plasma depletion in Mercury's subsolar magnetosheath. J. Geophys. Res. Space Phys. 118(11), 7181-7199 (2013). https://doi.org/10.1002/2013JA019244

K.H. Glassmeier, Currents in Mercury's Magnetosphere. Washington DC American Geophysical Union Geophysical Monograph Series, vol. 118 (2000), p. 371. https://doi.org/10.1029/GM118p0371

K.H. Glassmeier, H.U. Auster, D. Heyner, K. Okrafka, C. Carr, G. Berghofer, B.J. Anderson, A. Balogh, W. Baumjohann, P. Cargill, U. Christensen, M. Delva, M. Dougherty, K.H. Fornaçon, T.S. Horbury, E.A. Lucek, W. Magnes, M. Mandea, A. Matsuoka, M. Matsushima, U. Motschmann, R. Nakamura, Y. Narita, H. O’Brien, I. Richter, K. Schwingenschuh, H. Shibuya, J.A. Slavin, C. Sotin, B. Stoll, H. Tsunakawa, S. Vennerstrom, J. Vogt, T. Zhang, The fluxgate magnetometer of the BepiColombo Mercury Planetary Orbiter. Planet. Space Sci. 58(1-2), 287-299 (2010). https://doi.org/10.1016/ j.pss.2008.06.018

S.W. Good, R.J. Forsyth, Interplanetary coronal mass ejections observed by MESSENGER and Venus Express. Sol. Phys. 291(1), 239-263 (2016). https://doi.org/10.1007/s11207-015-0828-3. 1511.07749

B. Grieger, N. Ignatiev, D. Titov, Light scattering in Venus' atmosphere: a reanalysis of Venera 14 spectrophotometer data, in EAEJA, 9112 (2003)

E. Grün, N. Pailer, H. Fechtig, J. Kissel, Orbital and physical characteristics of micrometeoroids in the inner solar system as observed by Helios 1. Planet. Space Sci. 28(3), 333-349 (1980)

H. Grünwaldt, M. Neugebauer, M. Hilchenbach, P. Bochsler, D. Hovestadt, A. Bürgi, F.M. Ipavich, K.U. Reiche, W.I. Axford, H. Balsiger, A.B. Galvin, J. Geiss, F. Gliem, G. Gloeckler, K.C. Hsieh, R. Kallenbach, B. Klecker, S. Livi, M.A. Lee, G.G. Managadze, E. Marsch, E. Möbius, M. Scholer, M.I. Verigin, B. Wilken, P. Wurz, Venus tail ray observation near Earth. Geophys. Res. Lett. 24(10), 1163-1166 (1997). https://doi.org/10.1029/97GL01159

L. Guicking, K.H. Glassmeier, H.U. Auster, M. Delva, U. Motschmann, Y. Narita, T.L. Zhang, Low-frequency magnetic field fluctuations in Venus' solar wind interaction region: Venus Express observations. Ann. Geophys. 28(4), 951-967 (2010). https://doi.org/10.5194/angeo-28-951-2010

H. Gunell, E. Kallio, R. Jarvinen, P. Janhunen, M. Holmström, K. Dennerl, Simulations of solar wind charge exchange X-ray emissions at Venus. Geophys. Res. Lett. 34(3), L03107 (2007). https://doi.org/ 10.1029/2006GL028602

L.Z. Hadid, F. Sahraoui, K.H. Kiyani, A. Retinò, R. Modolo, P. Canu, A. Masters, M.K. Dougherty, Nature of the MHD and kinetic scale turbulence in the magnetosheath of Saturn: Cassini observations. Astrophys. J. Lett. 813(2), L29 (2015). https://doi.org/10.1088/2041-8205/813/2/L29. 1611.08245

J.E. Hansen, J. Hovenier, Interpretation of the polarization of Venus. J. Atmos. Sci. 31(4), 1137-1160 (1974)

A. Hasegawa, Drift mirror instability of the magnetosphere. Phys. Fluids 12, 2642-2650 (1969). https://doi.org/10.1063/1.1692407

A. Hasegawa, B.T. Tsurutani, Mirror mode expansion in planetary magnetosheaths: Bohm-like diffusion. Phys. Rev. Lett. 107(24), 245005 (2011). https://doi.org/10.1103/PhysRevLett.107.245005

R. Haus, D. Kappel, G. Arnold, Atmospheric thermal structure and cloud features in the southern hemisphere of Venus as retrieved from VIRTIS/VEX radiation measurements. Icarus 232, 232-248 (2014). https://doi.org/10.1016/j.icarus.2014.01.020

M. He, J. Vogt, D. Heyner, J. Zhong, Solar wind controls on Mercury's magnetospheric cusp. J. Geophys. Res. Space Phys. 122(6), 6150-6164 (2017). https://doi.org/10.1002/2016JA023687

J. He, B. Cui, L. Yang, C. Hou, L. Zhang, W.H. Ip, Y. Jia, C. Dong, D. Duan, Q. Zong et al., Encounter of Parker Solar Probe and a comet-like object during their perihelia: model predictions and measurements. ArXiv preprint (2020). arXiv:2012.00005

Heyner et al. (2021). This journal (for MAG instrument)

H. Hiesinger, J. Helbert, G. Alemanno, K. Bauch, M. D’Amore, A. Maturilli, A. Morlok, M. Reitze, C. Stangarone, A. Stojic et al., Studying the composition and mineralogy of the Hermean surface with the Mercury Radiometer and Thermal Infrared Spectrometer (MERTIS) for the BepiColombo mission: an update. Space Sci. Rev. 216(6), 1-37 (2020)

J. Hoffman, V. Oyama, U. Von Zahn, Measurements of the Venus lower atmosphere composition: a comparison of results. J. Geophys. Res. Space Phys. 85(A13), 7871-7881 (1980) 
L.L. Hood, Magnetic anomalies concentrated near and within Mercury's impact basins: early mapping and interpretation. J. Geophys. Res., Planets 121(6), 1016-1025 (2016). https://doi.org/10.1002/ 2016JE005048

L.L. Hood, J.S. Oliveira, V. Galluzzi, D.A. Rothery, Investigating sources of Mercury's crustal magnetic field: further mapping of MESSENGER magnetometer data. J. Geophys. Res., Planets 123(10), 2647-2666 (2018). https://doi.org/10.1029/2018JE005683

R. Howard, A. Vourlidas, R. Colaninno, C. Korendyke, S. Plunkett, M. Carter, D. Wang, N. Rich, S. Lynch, A. Thurn, D. Socker, A. Thernisien, D. Chua, The Solar Orbiter Heliospheric Imager (SoLoHI). Astron. Astrophys. 642, A13 (2019). https://doi.org/10.1051/0004-6361/201935202

S.Y. Huang, H.S. Fu, Z.G. Yuan, A. Vaivads, Y.V. Khotyaintsev, A. Retino, M. Zhou, D.B. Graham, K. Fujimoto, F. Sahraoui, X.H. Deng, B. Ni, Y. Pang, S. Fu, D.D. Wang, X. Zhou, Two types of whistler waves in the Hall reconnection region. J. Geophys. Res. Space Phys. 121(7), 6639-6646 (2016). https://doi.org/ 10.1002/2016JA022650

S.Y. Huang, L.Z. Hadid, F. Sahraoui, Z.G. Yuan, X.H. Deng, On the existence of the Kolmogorov inertial range in the terrestrial magnetosheath turbulence. Astrophys. J. Lett. 836(1), L10 (2017). https://doi.org/ 10.3847/2041-8213/836/1/L10. 1611.00199

S.Y. Huang, K. Jiang, Z.G. Yuan, F. Sahraoui, L.H. He, M. Zhou, H.S. Fu, X.H. Deng, J.S. He, D. Cao, X.D. Yu, D.D. Wang, J.L. Burch, C.J. Pollock, R.B. Torbert, Observations of the electron jet generated by secondary reconnection in the terrestrial magnetotail. Astrophys. J. 862(2), 144 (2018). https://doi.org/ $10.3847 / 1538-4357 / \mathrm{aacd} 4 \mathrm{c}$

S.Y. Huang, Q.Y. Wang, F. Sahraoui, Z.G. Yuan, Y.J. Liu, X.H. Deng, W.J. Sun, K. Jiang, S.B. Xu, X.D. Yu, Y.Y. Wei, J. Zhang, Analysis of turbulence properties in the Mercury plasma environment using MESSENGER observations. Astrophys. J. 891(2), 159 (2020). https://doi.org/10.3847/1538-4357/ab7349

R. Hueso, J. Peralta, I. Garate-Lopez, T.V. Bandos, A. Sánchez-Lavega, Six years of Venus winds at the upper cloud level from UV, visible and near infrared observations from VIRTIS on Venus Express. Planet. Space Sci. 113, 78-99 (2015). https://doi.org/10.1016/j.pss.2014.12.010

J. Huovelin, R. Vainio, E. Kilpua, A. Lehtolainen, S. Korpela, E. Esko, K. Muinonen, E. Bunce, A. Martindale, M. Grande et al., Solar Intensity X-ray and Particle Spectrometer SIXS: instrument design and first results. Space Sci. Rev. 216(5), 1-42 (2020)

K. Hurley, V. Pal'Shin, R. Aptekar, S. Golenetskii, D. Frederiks, E. Mazets, D. Svinkin, M. Briggs, V. Connaughton, C. Meegan et al., The interplanetary network supplement to the Fermi GBM catalog of cosmic gamma-ray bursts. Astrophys. J. Suppl. Ser. 207(2), 39 (2013)

V. Iafolla, S. Nozzoli, Italian Spring Accelerometer (ISA) a high sensitive accelerometer for "BepiColombo" ESA cornerstone. Planet. Space Sci. 49(14-15), 1609-1617 (2001)

V. Iafolla, D.M. Lucchesi, S. Nozzoli, F. Santoli, ISA accelerometer onboard the Mercury Planetary Orbiter: error budget. Celest. Mech. Dyn. Astron. 97(3), 165-187 (2007). https://doi.org/10.1007/ s10569-006-9059-0

V. Iafolla, E. Fiorenza, C. Lefevre, A. Morbidini, S. Nozzoli, R. Peron, M. Persichini, A. Reale, F. Santoli, Italian Spring Accelerometer (ISA): a fundamental support to BepiColombo radio science experiments. Planet. Space Sci. 58(1-2), 300-308 (2010)

V. Iafolla, E. Fiorenza, C. Lefevre, D.M. Lucchesi, M. Lucente, C. Magnafico, R. Peron, F. Santoli, The BepiColombo ISA accelerometer: ready for launch, in 2016 IEEE Metrology for Aerospace (MetroAeroSpace). IEEE (2016), pp. 538-544

Iess et al. (2021). This journal (for MORE experiment)

N.I. Ignatiev, D.V. Titov, G. Piccioni, P. Drossart, W.J. Markiewicz, V. Cottini, T. Roatsch, M. Almeida, N. Manoel, Altimetry of the Venus cloud tops from the Venus Express observations. J. Geophys. Res., Planets 114(E5), E00B43 (2009). https://doi.org/10.1029/2008JE003320

S.M. Imber, J.A. Slavin, MESSENGER observations of magnetotail loading and unloading: implications for substorms at Mercury. J. Geophys. Res. Space Phys. 122(11), 11,402-11,412 (2017). https://doi.org/ 10.1002/2017JA024332

S.M. Imber, J.A. Slavin, S.A. Boardsen, B.J. Anderson, H. Korth, R.L. McNutt, S.C. Solomon, MESSENGER observations of large dayside flux transfer events: do they drive Mercury's substorm cycle? J. Geophys. Res. Space Phys. 119(7), 5613-5623 (2014). https://doi.org/10.1002/2014JA019884

K. Iwai, D. Shiota, M. Tokumaru, K. Fujiki, M. Den, Y. Kubo, Development of a coronal mass ejection arrival time forecasting system using interplanetary scintillation observations. Earth Planets Space 71(1), 39 (2019). https://doi.org/10.1186/s40623-019-1019-5. 1903.11769

K. Iwai, S. Yashiro, N.V. Nitta, Y. Kubo, Spectral structures of type II solar radio bursts and solar energetic particles. Astrophys. J. 888(1), 50 (2020). https://doi.org/10.3847/1538-4357/ab57ff. 1911.05897

B.V. Jackson, P.P. Hick, A. Buffington, M.M. Bisi, J.M. Clover, M. Tokumaru, M. Kojima, K. Fujiki, Threedimensional reconstruction of heliospheric structure using iterative tomography: a review. J. Atmos. Sol.-Terr. Phys. 73(10), 1214-1227 (2011). https://doi.org/10.1016/j.jastp.2010.10.007 
B.V. Jackson, D. Odstrcil, H.S. Yu, P.P. Hick, A. Buffington, J.C. Mejia-Ambriz, J. Kim, S. Hong, Y. Kim, J. Han, M. Tokumaru, The UCSD kinematic IPS solar wind boundary and its use in the ENLIL 3-D MHD prediction model. Space Weather 13(2), 104-115 (2015). https://doi.org/10.1002/2014SW001130

M. Jeřáb, Z. Němeček, J. Šafránková, K. Jelínek, J. Měrka, Improved bow shock model with dependence on the IMF strength. Planet. Space Sci. 53(1-3), 85-93 (2005). https://doi.org/10.1016/j.pss.2004.09.032

K.L. Jessup, E. Marcq, F. Mills, A. Mahieux, S. Limaye, C. Wilson, M. Allen, J.L. Bertaux, W. Markiewicz, T. Roman, A.C. Vandaele, V. Wilquet, Y. Yung, Coordinated Hubble Space Telescope and Venus Express observations of Venus' upper cloud deck. Icarus 258, 309-336 (2015). https://doi.org/10.1016/ j.icarus.2015.05.027

X. Jia, J.A. Slavin, G. Poh, G.A. DiBraccio, G. Toth, Y. Chen, J.M. Raines, T.I. Gombosi, MESSENGER observations and global simulations of highly compressed magnetosphere events at Mercury. J. Geophys. Res. Space Phys. 124(1), 229-247 (2019). https://doi.org/10.1029/2018JA026166

S.R. Kane, G. Arney, D. Crisp, S. Domagal-Goldman, L.S. Glaze, C. Goldblatt, D. Grinspoon, J.W. Head, A. Lenardic, C. Unterborn et al., Venus as a laboratory for exoplanetary science. J. Geophys. Res., Planets 124(8), 2015-2028 (2019)

T. Karlsson, Y. Kasaba, J.E. Wahlund, P. Henri, L. Bylander, W. Puccio, S.E. Jansson, L. Åhlen, E. Kallio, H. Kojima et al., The MEFISTO and WPT electric field sensors of the plasma wave investigation on the BepiColombo Mio spacecraft. Space Sci. Rev. 216(8), 1-24 (2020). https://doi.org/10.1007/s11214-020-00760-0

Y. Kasaba, T. Takashima, S. Matsuda, S. Eguchi, M. Endo, T. Miyabara, M. Taeda, Y. Kuroda, Y. Kasahara, T. Imachi et al., Mission data processor aboard the BepiColombo Mio spacecraft: design and scientific operation concept. Space Sci. Rev. 216(3), 1-19 (2020). https://doi.org/10.1007/s11214-020-00692-9

R.M. Killen, T.A. Bida, T.H. Morgan, The calcium exosphere of Mercury. Icarus 173(2), 300-311 (2005). https://doi.org/10.1016/j.icarus.2004.08.022

E.K.J. Kilpua, A. Isavnin, A. Vourlidas, H.E.J. Koskinen, L. Rodriguez, On the relationship between interplanetary coronal mass ejections and magnetic clouds. Ann. Geophys. 31(7), 1251-1265 (2013). https://doi.org/10.5194/angeo-31-1251-2013

E.K.J. Kilpua, A. Balogh, R. von Steiger, Y.D. Liu, Geoeffective properties of solar transients and stream interaction regions. Space Sci. Rev. 212(3-4), 1271-1314 (2017). https://doi.org/10.1007/ s11214-017-0411-3

E.K.J. Kilpua, S.W. Good, E. Palmerio, E. Asvestari, E. Lumme, M. Ala-Lahti, M.M.H. Kalliokoski, D.E. Morosan, J. Pomoell, D.J. Price, J. Magdalenić, S. Poedts, Y. Futaana, Multipoint observations of the June 2012 interacting interplanetary flux ropes. Front. Astron. Space Sci. 6, 50 (2019). https://doi.org/ $10.3389 /$ fspas. 2019.00050

L.W. Klein, L.F. Burlaga, Interplanetary magnetic clouds at 1 AU. J. Geophys. Res. 87(A2), 613-624 (1982). https://doi.org/10.1029/JA087iA02p00613

A. Kliore, G.S. Levy, D.L. Cain, G. Fjeldbo, S.I. Rasool, Atmosphere and ionosphere of Venus from the Mariner V S-band radio occultation measurement. Science 158(3809), 1683-1688 (1967). https:// doi.org/10.1126/science.158.3809.1683

R.G. Knollenberg, D.M. Hunten, The microphysics of the clouds of Venus - results of the Pioneer Venus particle size spectrometer experiment. J. Geophys. Res. 85, 8039-8058 (1980). https://doi.org/ 10.1029/JA085iA13p08039

W.C. Knudsen, K.L. Miller, The Venus transterminator ion flux at solar maximum. J. Geophys. Res. 97(A11), 17165-17167 (1992). https://doi.org/10.1029/92JA01460

W.C. Knudsen, K. Spenner, K.L. Miller, V. Novak, Transport of ionospheric $\mathrm{O}^{+}$ions across the Venus terminator and implications. J. Geophys. Res. 85, 7803-7810 (1980). https://doi.org/10.1029/ JA085iA13p07803

W.C. Knudsen, P.M. Banks, K.L. Miller, A new concept of plasma motion and planetary magnetic field for Venus. Geophys. Res. Lett. 9(7), 765-768 (1982). https://doi.org/10.1029/GL009i007p00765

M. Kobayashi, H. Shibata, K. Nogami, M. Fujii, S. Hasegawa, M. Hirabayashi, T. Hirai, T. Iwai, H. Kimura, T. Miyachi et al., Mercury Dust Monitor (MDM) onboard the Mio orbiter of the BepiColombo mission. Space Sci. Rev. 216(8), 1-18 (2020). https://doi.org/10.1007/s11214-020-00775-7

P. Kollmann, P.C. Brandt, G. Collinson, Z.J. Rong, Y. Futaana, T.L. Zhang, Properties of planetward ion flows in Venus' magnetotail. Icarus 274, 73-82 (2016). https://doi.org/10.1016/j.icarus.2016.02.053

R.P. Kornfeld, B.W. Arnold, M.A. Gross, N.T. Dahya, W.M. Klipstein, P.F. Gath, S. Bettadpur, GRACE-FO: the gravity recovery and climate experiment follow-on mission. J. Spacecr. Rockets 56(3), 931-951 (2019). https://doi.org/10.2514/1.A34326

H. Korth, B.J. Anderson, J.M. Raines, J.A. Slavin, T.H. Zurbuchen, C.L. Johnson, M.E. Purucker, R.M. Winslow, S.C. Solomon, R.L. McNutt Jr., Plasma pressure in Mercury's equatorial magnetosphere derived from MESSENGER magnetometer observations. Geophys. Res. Lett. 38(22), L22201 (2011) 
H. Korth, C.L. Johnson, L. Philpott, N.A. Tsyganenko, B.J. Anderson, A dynamic model of Mercury's magnetospheric magnetic field. Geophys. Res. Lett. 44(20), 10-147 (2017)

T. Kouyama, T. Imamura, M. Taguchi, T. Fukuhara, T.M. Sato, A. Yamazaki, M. Futaguchi, S. Murakami, G.L. Hashimoto, M. Ueno, N. Iwagami, S. Takagi, M. Takagi, K. Ogohara, H. Kashimura, T. Horinouchi, N. Sato, M. Yamada, Y. Yamamoto, S. Ohtsuki, K. Sugiyama, H. Ando, M. Takamura, T. Yamada, T. Satoh, M. Nakamura, Topographical and local time dependence of large stationary gravity waves observed at the cloud top of Venus. Geophys. Res. Lett. 44(24), 12,098-12,105 (2017). https://doi.org/10.1002/2017GL075792

T. Kouyama, M. Taguchi, T. Fukuhara, T. Imamura, T. Horinouchi, T.M. Sato, S. Murakami, G.L. Hashimoto, Y.J. Lee, M. Futaguchi, T. Yamada, M. Akiba, T. Satoh, M. Nakamura, Global structure of thermal tides in the upper cloud layer of Venus revealed by LIR on board Akatsuki. Geophys. Res. Lett. 46(16), 9457-9465 (2019). https://doi.org/10.1029/2019GL083820. 1905.08947

A. Kozyrev, I. Mitrofanov, J. Benkhoff, A. Owens, F. Quarati, M. Litvak, A. Malakhov, M. Mokrousov, G. Timoshenko, V. Shvetsov, Next generation of scintillation detector based on cerium bromide crystal for space application in the gamma-ray spectrometer of the Mercurian gamma-ray and neutron spectrometer. Instrum. Exp. Tech. 59(4), 569-577 (2016)

S.M. Krimigis, D.G. Mitchell, D.H. Hamilton, S. Livi, J. Dandouras, Preliminary results from MIMI observations during Cassini's Venus-2 flyby on June 24, 1999, in AAS/Division for Planetary Sciences Meeting Abstracts \#31, AAS/Division for Planetary Sciences Meeting Abstracts, 64.04 (1999)

H. Lammer, H.I.M. Lichtenegger, H.K. Biernat, N.V. Erkaev, I.L. Arshukova, C. Kolb, H. Gunell, A. Lukyanov, M. Holmstrom, S. Barabash, T.L. Zhang, W. Baumjohann, Loss of hydrogen and oxygen from the upper atmosphere of Venus. Planet. Space Sci. 54(13-14), 1445-1456 (2006). https://doi.org/ 10.1016/j.pss.2006.04.022

D. Lario, R.Y. Kwon, A. Vourlidas, N.E. Raouafi, D.K. Haggerty, G.C. Ho, B.J. Anderson, A. Papaioannou, R. Gómez-Herrero, N. Dresing, P. Riley, Longitudinal properties of a widespread solar energetic particle event on 2014 February 25: evolution of the associated CME shock. Astrophys. J. 819(1), 72 (2016). https://doi.org/10.3847/0004-637X/819/1/72

G. Le, P.J. Chi, X. Blanco-Cano, S. Boardsen, J.A. Slavin, B.J. Anderson, H. Korth, Upstream ultra-low frequency waves in Mercury's foreshock region: MESSENGER magnetic field observations. J. Geophys. Res. Space Phys. 118(6), 2809-2823 (2013)

F. Leblanc, A. Doressoundiram, N. Schneider, V. Mangano, A. López Ariste, C. Lemen, B. Gelly, C. Barbieri, G. Cremonese, High latitude peaks in Mercury's sodium exosphere: spectral signature using THEMIS solar telescope. Geophys. Res. Lett. 35(18), L18204 (2008). https://doi.org/10.1029/2008GL035322

F. Leblanc, A. Doressoundiram, N. Schneider, S. Massetti, M. Wedlund, A. López Ariste, C. Barbieri, V. Mangano, G. Cremonese, Short-term variations of Mercury's Na exosphere observed with very high spectral resolution. Geophys. Res. Lett. 36(7), L07201 (2009). https://doi.org/10.1029/2009GL038089

Y.J. Lee, K.L. Jessup, S. Perez-Hoyos, D.V. Titov, S. Lebonnois, J. Peralta, T. Horinouchi, T. Imamura, S. Limaye, E. Marcq, M. Takagi, A. Yamazaki, M. Yamada, S. Watanabe, S.y. Murakami, K. Ogohara, W.M. McClintock, G. Holsclaw, A. Roman, Long-term variations of Venus's $365 \mathrm{~nm}$ albedo observed by Venus Express, Akatsuki, MESSENGER, and the Hubble Space Telescope. Astron. J. 158(3), 126 (2019). https://doi.org/10.3847/1538-3881/ab3120. 1907.09683

Y. Lee, A.G. Muñoz, T. Imamura, M. Yamada, T. Satoh, A. Yamazaki, S. Watanabe, Brightness modulations of our nearest terrestrial planet Venus reveal atmospheric super-rotation rather than surface features. Nat. Commun. 11(1), 1-8 (2020)

J. Lilensten, A.J. Coates, V. Dehant, T. Dudok de Wit, R.B. Horne, F. Leblanc, J. Luhmann, E. Woodfield, M. Barthélemy, What characterizes planetary space weather? Astron. Astrophys. Rev. 22, 79 (2014). https://doi.org/10.1007/s00159-014-0079-6

S.S. Limaye, S. Lebonnois, A. Mahieux, M. Pätzold, S. Bougher, S. Bruinsma, S. Chamberlain, R.T. Clancy, J.C. Gérard, G. Gilli, D. Grassi, R. Haus, M. Herrmann, T. Imamura, E. Kohler, P. Krause, A. Migliorini, F. Montmessin, C. Pere, M. Persson, A. Piccialli, M. Rengel, A. Rodin, B. Sandor, M. Sornig, H. Svedhem, S. Tellmann, P. Tanga, A.C. Vandaele, T. Widemann, C.F. Wilson, I. Müller-Wodarg, L. Zasova, The thermal structure of the Venus atmosphere: intercomparison of Venus Express and ground based observations of vertical temperature and density profiles. Icarus 294, 124-155 (2017). https://doi.org/10.1016/j.icarus.2017.04.020

S.S. Limaye, D. Grassi, A. Mahieux, A. Migliorini, S. Tellmann, D. Titov, Venus atmospheric thermal structure and radiative balance. Space Sci. Rev. 214(5), 102 (2018). https://doi.org/10.1007/ s11214-018-0525-2

M. Livshits, I. Zimovets, D. Golovin, B. Nizamov, V. Vybornov, I. Mitrofanov, A. Kozyrev, M. Litvak, A. Sanin, V. Tretyakov, Catalog of hard X-ray solar flares detected with Mars Odyssey/HEND from the Mars orbit in 2001-2016. Astron. Rep. 61(9), 791-804 (2017) 
D.M. Lucchesi, V. Iafolla, The non-gravitational perturbations impact on the BepiColombo radio science experiment and the key rôle of the ISA accelerometer: direct solar radiation and albedo effects. Celest. Mech. Dyn. Astron. 96, 99-127 (2006). https://doi.org/10.1007/s10569-006-9034-9

N. Lugaz, M. Temmer, Y. Wang, C.J. Farrugia, The interaction of successive coronal mass ejections: a review. Sol. Phys. 292(4), 64 (2017). https://doi.org/10.1007/s11207-017-1091-6. 1612.02398

M. Luginin, A. Fedorova, D. Belyaev, F. Montmessin, V. Wilquet, O. Korablev, J.L. Bertaux, A.C. Vandaele, Aerosol properties in the upper haze of Venus from SPICAV IR data. Icarus 277, 154-170 (2016). https://doi.org/10.1016/j.icarus.2016.05.008

J.G. Luhmann, The inner magnetosheath of Venus: an analogue for Earth? J. Geophys. Res. 100(A7), 1203512046 (1995). https://doi.org/10.1029/94JA02862

J.G. Luhmann, S.A. Ledvina, D. Odstrcil, M.J. Owens, X.P. Zhao, Y. Liu, P. Riley, Cone model-based SEP event calculations for applications to multipoint observations. Adv. Space Res. 46(1), 1-21 (2010). https://doi.org/10.1016/j.asr.2010.03.011

J.G. Luhmann, C. Dong, Y. Ma, S.M. Curry, D. Mitchell, J. Espley, J. Connerney, J. Halekas, D.A. Brain, B.M. Jakosky, C. Mazelle, Implications of MAVEN Mars near-wake measurements and models. Geophys. Res. Lett. 42(21), 9087-9094 (2015). https://doi.org/10.1002/2015GL066122

P. Machado, T. Widemann, J. Peralta, R. Gonçalves, J.F. Donati, D. Luz, Venus cloud-tracked and Doppler velocimetry winds from CFHT/ESPaDOnS and Venus Express/VIRTIS in April 2014. Icarus 285, 8-26 (2017). https://doi.org/10.1016/j.icarus.2016.12.017

D. Magurno, T. Maestri, D. Grassi, G. Piccioni, G. Sindoni, Retrieval of Venus' cloud parameters from VIRTIS nightside spectra in the latitude band $25^{\circ}-55^{\circ}$ N. Planet. Space Sci. 144, 16-31 (2017). https:// doi.org/10.1016/j.pss.2017.06.004

W. Manchester, E.K.J. Kilpua, Y.D. Liu, N. Lugaz, P. Riley, T. Török, B. Vršnak, The physical processes of CME/ICME evolution. Space Sci. Rev. 212(3-4), 1159-1219 (2017). https://doi.org/10.1007/ s11214-017-0394-0

V. Mangano, S. Massetti, A. Milillo, A. Mura, S. Orsini, F. Leblanc, Dynamical evolution of sodium anisotropies in the exosphere of Mercury. Planet. Space Sci. 82, 1-10 (2013). https://doi.org/10.1016/ j.pss.2013.03.002

V. Mangano, S. Massetti, A. Milillo, C. Plainaki, S. Orsini, R. Rispoli, F. Leblanc, THEMIS Na exosphere observations of Mercury and their correlation with in-situ magnetic field measurements by MESSENGER. Planet. Space Sci. 115, 102-109 (2015). https://doi.org/10.1016/j.pss.2015.04.001

E. Marcq, K. Lea Jessup, L. Baggio, T. Encrenaz, Y.J. Lee, F. Montmessin, D. Belyaev, O. Korablev, J.L. Bertaux, Climatology of $\mathrm{SO}_{2}$ and UV absorber at Venus' cloud top from SPICAV-UV nadir dataset. Icarus 335, 113368 (2020). https://doi.org/10.1016/j.icarus.2019.07.002

W. Markiewicz, D. Titov, S. Limaye, H.U. Keller, N. Ignatiev, R. Jaumann, N. Thomas, H. Michalik, R. Moissl, P. Russo, Morphology and dynamics of the upper cloud layer of Venus. Nature 450(7170), 633-636 (2007)

C. Martinecz, A. Boesswetter, M. Fränz, E. Roussos, J. Woch, N. Krupp, E. Dubinin, U. Motschmann, S. Wiehle, S. Simon, S. Barabash, R. Lundin, T.L. Zhang, H. Lammer, H. Lichtenegger, Y. Kulikov, Plasma environment of Venus: comparison of Venus Express ASPERA-4 measurements with 3-D hybrid simulations. J. Geophys. Res., Planets 114(E9), E00B30 (2009). https://doi.org/10.1029/2008JE003174

S. Massetti, V. Mangano, A. Milillo, A. Mura, S. Orsini, C. Plainaki, Short-term observations of doublepeaked Na emission from Mercury's exosphere. Geophys. Res. Lett. 44(7), 2970-2977 (2017). https://doi.org/10.1002/2017GL073090

K. Masunaga, K. Seki, N. Terada, F. Tsuchiya, T. Kimura, K. Yoshioka, G. Murakami, A. Yamazaki, M. Kagitani, C. Tao, A. Fedorov, Y. Futaana, T.L. Zhang, D. Shiota, F. Leblanc, J.Y. Chaufray, I. Yoshikawa, Periodic variations of oxygen EUV dayglow in the upper atmosphere of Venus: Hisaki/EXCEED observations. J. Geophys. Res., Planets 120(12), 2037-2052 (2015). https://doi.org/10.1002/2015JE004849

M.B. McElroy, D.F. Strobel, Models for the nighttime Venus ionosphere. J. Geophys. Res. 74(5), 1118 (1969). https://doi.org/10.1029/JA074i005p01118

S. McKenna-Lawlor, V.V. Afonin, K.I. Gringauz, E. Keppler, E. Kirsch, A. Richter, M. Witte, D. O’Sullivan, A. Thompson, A.J. Somogyi, L. Szabo, A. Varga, The low energy particle detector sled ( $30 \mathrm{keV}-3.2$ $\mathrm{MeV}$ ) and its performance on the Phobos mission to Mars and its moons. Nucl. Instrum. Methods Phys. Res., Sect. A 290(1), 217-222 (1990). https://doi.org/10.1016/0168-9002(90)90364-C

S. McKenna-Lawlor, E. Kallio, R. Jarvinen, V.V. Afonin, Magnetic shadowing of high energy ions at Mars and how this effect can be simulated using a hybrid model. Earth Planets Space 64(2), 247-256 (2012). https://doi.org/10.5047/eps.2011.06.039

J. McNutt, L. Ralph, S.C. Solomon, D.G. Grant, E.J. Finnegan, P.D. Bedini, The MESSENGER mission to Mercury: status after the Venus flybys. Acta Astronaut. 63, 68-73 (2008). https://doi.org/ 10.1016/j.actaastro.2007.12.062 
A. Mignone, M. Flock, M. Stute, S.M. Kolb, G. Muscianisi, A conservative orbital advection scheme for simulations of magnetized shear flows with the PLUTO code. Astron. Astrophys. 545, A152 (2012). https://doi.org/10.1051/0004-6361/201219557. 1207.2955

A. Milani, A.M. Nobili, P. Farinella, Non-gravitational Perturbations and Satellite Geodesy (Adam Hilger Ltd., Bristol, 1987)

A. Milillo, M. Fujimoto, G. Murakami, J. Benkhoff, J. Zender, S. Aizawa, M. Dósa, L. Griton, D. Heyner, G. Ho et al., Investigating Mercury's environment with the two-spacecraft BepiColombo mission. Space Sci. Rev. 216(5), 1-78 (2020)

K.L. Miller, W.C. Knudsen, Spatial and temporal variations of the ion velocity measured in the Venus ionosphere. Adv. Space Res. 7(12), 107-110 (1987). https://doi.org/10.1016/0273-1177(87)90207-9

K.L. Miller, R.C. Whitten, Ion dynamics in the Venus ionosphere. Space Sci. Rev. 55(1-4), 165-199 (1991). https://doi.org/10.1007/BF00177137

K.L. Miller, W.C. Knudsen, K. Spenner, R.C. Whitten, V. Novak, Solar zenith angle dependence of ionospheric ion and electron temperatures and density on Venus. J. Geophys. Res. Space Phys. 85(A13), 7759-7764 (1980)

L.I. Miroshnichenko, Retrospective analysis of GLEs and estimates of radiation risks. J. Space Weather Space Clim. 8, A52 (2018). https://doi.org/10.1051/swsc/2018042

I. Mitrofanov, A. Kozyrev, A. Konovalov, M. Litvak, A. Malakhov, M. Mokrousov, A. Sanin, V. Tret'ykov, A. Vostrukhin, Y.I. Bobrovnitskij et al., The Mercury Gamma and Neutron Spectrometer (MGNS) on board the Planetary Orbiter of the BepiColombo mission. Planet. Space Sci. 58(1-2), 116-124 (2010)

Mitrofanov et al. (2021). This journal (for MGNS instrument)

M. Miyamoto, T. Imamura, M. Tokumaru, H. Ando, H. Isobe, A. Asai, D. Shiota, T. Toda, B. Häusler, M. Pätzold et al., Radial distribution of compressive waves in the solar corona revealed by Akatsuki radio occultation observations. Astrophys. J. 797(1), 51 (2014)

J. Müller, S. Simon, Y.-C. Wang, U. Motschmann, D. Heyner, J. Schüle, W.-H. Ip, G. Kleindienst, G.J. Pringle, Origin of Mercury's double magnetopause: 3D hybrid simulation study with AIKEF. Icarus 218(1), 666-687 (2012). https://doi.org/10.1016/j.icarus.2011.12.028

F. Montmessin, J.L. Bertaux, F. Lefèvre, E. Marcq, D. Belyaev, J.C. Gérard, O. Korablev, A. Fedorova, V. Sarago, A.C. Vandaele, A layer of ozone detected in the nightside upper atmosphere of Venus. Icarus 216(1), 82-85 (2011). https://doi.org/10.1016/j.icarus.2011.08.010

G. Murakami, H. Hayakawa, H. Ogawa, S. Matsuda, T. Seki, Y. Kasaba, Y. Saito, I. Yoshikawa, M. Kobayashi, W. Baumjohann et al., Mio-first comprehensive exploration of Mercury's space environment: mission overview. Space Sci. Rev. 216(7), 1-25 (2020). https://doi.org/10.1007/s11214-020-00733-3

A.F. Nagy, T.E. Cravens, J.H. Yee, A.I.F. Stewart, Hot oxygen atoms in the upper atmosphere of Venus. Geophys. Res. Lett. 8(6), 629-632 (1981). https://doi.org/10.1029/GL008i006p00629

M. Nakamura, T. Imamura, N. Ishii, T. Abe, Y. Kawakatsu, C. Hirose, T. Satoh, M. Suzuki, M. Ueno, A. Yamazaki, N. Iwagami, S. Watanabe, M. Taguchi, T. Fukuhara, Y. Takahashi, M. Yamada, M. Imai, S. Ohtsuki, K. Uemizu, G.L. Hashimoto, M. Takagi, Y. Matsuda, K. Ogohara, N. Sato, Y. Kasaba, T. Kouyama, N. Hirata, R. Nakamura, Y. Yamamoto, T. Horinouchi, M. Yamamoto, Y.Y. Hayashi, H. Kashimura, S. Ki, T. Sakanoi, H. Ando, S.y. Murakami, T.M. Sato, S. Takagi, K. Nakajima, J. Peralta, Y.J. Lee, J. Nakatsuka, T. Ichikawa, K. Inoue, T. Toda, H. Toyota, S. Tachikawa, S. Narita, T. Hayashiyama, A. Hasegawa, Y. Kamata, AKATSUKI returns to Venus. Earth Planets Space 68(1), 75 (2016). https://doi.org/10.1186/s40623-016-0457-6

G. Napoletano, R. Forte, D. Del Moro, E. Pietropaolo, L. Giovannelli, F. Berrilli, A probabilistic approach to the drag-based model. J. Space Weather Space Clim. 8, A11 (2018)

Y. Narita, S.P. Gary, S. Saito, K.H. Glassmeier, U. Motschmann, Dispersion relation analysis of solar wind turbulence. Geophys. Res. Lett. 38(5), L05101 (2011). https://doi.org/10.1029/2010GL046588

T. Navarro, G. Schubert, S. Lebonnois, Atmospheric mountain wave generation on Venus and its influence on the solid planet's rotation rate. Nat. Geosci. 11(7), 487-491 (2018). https://doi.org/10.1038/ s41561-018-0157-X

N.F. Ness, K.W. Behannon, R.P. Lepping, Y.C. Whang, The magnetic field of Mercury, 1. J. Geophys. Res. 80(19), 2708 (1975). https://doi.org/10.1029/JA080i019p02708

T. NordströM, G. Stenberg, H. Nilsson, S. Barabash, T.L. Zhang, Venus ion outflow estimates at solar minimum: influence of reference frames and disturbed solar wind conditions. J. Geophys. Res. Space Phys. 118(6), 3592-3601 (2013). https://doi.org/10.1002/jgra.50305

D. Odstrcil, Modeling 3-D solar wind structure. Adv. Space Res. 32(4), 497-506 (2003). https://doi.org/ 10.1016/S0273-1177(03)00332-6

D. Odstrcil, V.J. Pizzo, Distortion of the interplanetary magnetic field by three-dimensional propagation of coronal mass ejections in a structured solar wind. J. Geophys. Res. 104, 28225-28240 (1999). https://doi.org/10.1029/1999JA900319 
D. Odstrcil, V. Pizzo, C. Arge, Propagation of the 12 May 1997 interplanetary coronal mass ejection in evolving solar wind structures. J. Geophys. Res. Space Phys. 110(A2), A02106 (2005)

J.S. Oliveira, B. Langlais, M.A. Pais, H. Amit, A modified Equivalent Source Dipole method to model partially distributed magnetic field measurements, with application to Mercury. J. Geophys. Res., Planets 120(6), 1075-1094 (2015). https://doi.org/10.1002/2014JE004734

J.S. Oliveira, L.L. Hood, B. Langlais, Constraining the early history of Mercury and its core dynamo by studying the crustal magnetic field. J. Geophys. Res., Planets 124(9), 2382-2396 (2019). https://doi.org/10.1029/2019JE005938

S. Orsini, V. Mangano, A. Milillo, C. Plainaki, A. Mura, J. Raines, E. De Angelis, R. Rispoli, F. Lazzarotto, A. Aronica, Mercury sodium exospheric emission as a proxy for solar perturbations transit. Sci. Rep. 8, 928 (2018). https://doi.org/10.1038/s41598-018-19163-X

J. Peralta, A. Sánchez-Lavega, M.A. López-Valverde, D. Luz, P. Machado, Venus's major cloud feature as an equatorially trapped wave distorted by the wind. Geophys. Res. Lett. 42(3), 705-711 (2015). https://doi.org/10.1002/2014GL062280

J. Peralta, Y.J. Lee, R. Hueso, R.T. Clancy, B.J. Sandor, A. Sánchez-Lavega, E. Lellouch, M. Rengel, P. Machado, M. Omino, A. Piccialli, T. Imamura, T. Horinouchi, S. Murakami, K. Ogohara, D. Luz, D. Peach, Venus's winds and temperatures during the MESSENGER's flyby: an approximation to a three-dimensional instantaneous state of the atmosphere. Geophys. Res. Lett. 44(8), 3907-3915 (2017). https://doi.org/10.1002/2017GL072900

S. Pérez-Hoyos, A. Sánchez-Lavega, A. García-Muñoz, P.G.J. Irwin, J. Peralta, G. Holsclaw, W.M. McClintock, J.F. Sanz-Requena, Venus upper clouds and the UV absorber from MESSENGER/MASCS observations. J. Geophys. Res., Planets 123(1), 145-162 (2018). https://doi.org/10.1002/2017JE005406. 1801.03820

M. Persson, Y. Futaana, A. Fedorov, H. Nilsson, M. Hamrin, S. Barabash, $\mathrm{H}^{+} / \mathrm{O}^{+}$escape rate ratio in the Venus magnetotail and its dependence on the solar cycle. Geophys. Res. Lett. 45(20), 10,805-10,811 (2018). https://doi.org/10.1029/2018GL079454

L.C. Philpott, C.L. Johnson, R.M. Winslow, B.J. Anderson, H. Korth, M.E. Purucker, S.C. Solomon, Constraints on the secular variation of Mercury's magnetic field from the combined analysis of MESSENGER and Mariner 10 data. Geophys. Res. Lett. 41(19), 6627-6634 (2014). https://doi.org/ 10.1002/2014GL061401

A. Piccialli, F. Montmessin, D. Belyaev, A. Mahieux, A. Fedorova, E. Marcq, J.L. Bertaux, S. Tellmann, A.C. Vandaele, O. Korablev, Thermal structure of Venus nightside upper atmosphere measured by stellar occultations with SPICAV/Venus Express. Planet. Space Sci. 113, 321-335 (2015). https://doi.org/10.1016/j.pss.2014.12.009

C.M. Pieters, J.N. Goswami, R.N. Clark, M. Annadurai, J. Boardman, B. Buratti, J.P. Combe, M.D. Dyar, R. Green, J.W. Head, C. Hibbitts, M. Hicks, P. Isaacson, R. Klima, G. Kramer, S. Kumar, E. Livo, S. Lundeen, E. Malaret, T. McCord, J. Mustard, J. Nettles, N. Petro, C. Runyon, M. Staid, J. Sunshine, L.A. Taylor, S. Tompkins, P. Varanasi, Character and spatial distribution of $\mathrm{OH} / \mathrm{H}_{2} \mathrm{O}$ on the surface of the Moon seen by $\mathrm{M}^{3}$ on Chandrayaan-1. Science 326(5952), 568 (2009). https://doi.org/10.1126/ science. 1178658

C. Plainaki, J. Lilensten, A. Radioti, M. Andriopoulou, A. Milillo, T.A. Nordheim, I. Dandouras, A. Coustenis, D. Grassi, V. Mangano, S. Massetti, S. Orsini, A. Lucchetti, Planetary space weather: scientific aspects and future perspectives. J. Space Weather Space Clim. 6, A31 (2016). https://doi.org/ $10.1051 /$ swsc/2016024

G. Poh, J.A. Slavin, X. Jia, J.M. Raines, S.M. Imber, W.J. Sun, D.J. Gershman, G.A. DiBraccio, K.J. Genestreti, A.W. Smith, Coupling between Mercury and its nightside magnetosphere: cross-tail current sheet asymmetry and substorm current wedge formation. J. Geophys. Res. Space Phys. 122(8), 8419-8433 (2017). https://doi.org/10.1002/2017JA024266

J. Pomoell, S. Poedts, EUHFORIA: European heliospheric forecasting information asset. J. Space Weather Space Clim. 8, A35 (2018). https://doi.org/10.1051/swsc/2018020

A.E. Potter, R.M. Killen, Observations of the sodium tail of Mercury. Icarus 194(1), 1-12 (2008). https://doi.org/10.1016/j.icarus.2007.09.023

A. Potter, T. Morgan, Discovery of sodium in the atmosphere of Mercury. Science 229(4714), 651-653 (1985)

A.E. Potter, T.H. Morgan, Evidence for magnetospheric effects on the sodium atmosphere of Mercury. Science 248(4957), 835-838 (1990). https://doi.org/10.1126/science.248.4957.835

E. Quémerais, J.Y. Chaufray, D. Koutroumpa, F. Leblanc, A. Reberac, B. Lustrement, C. Montaron, J.F. Mariscal, N. Rouanet, I. Yoshikawa et al., Phebus on Bepi-Colombo: post-launch update and instrument performance. Space Sci. Rev. 216(4), 67 (2020) 
R. Reasenberg, I. Shapiro, P. MacNeil, R. Goldstein, J. Breidenthal, J. Brenkle, D. Cain, T. Kaufman, T. Komarek, A. Zygielbaum, Viking relativity experiment-verification of signal retardation by solar gravity. Astrophys. J. 234, L219-L221 (1979)

C. Reigber, P. Schwintzer, R. Stubenvoll, R. Schmidt, F. Flechtner, U. Meyer, R. König, K.H. Neumayer, C. Förste, F. Barthelmes et al., A high resolution global gravity field model combining champ and grace satellite mission and surface data: Eigen-cg01c. Scientific Technical Report (2006)

I.G. Richardson, Solar wind stream interaction regions throughout the heliosphere. Living Rev. Sol. Phys. 15(1), 1 (2018). https://doi.org/10.1007/s41116-017-0011-z

A.C. Richie-Halford, L. Iess, P. Tortora, J.W. Armstrong, S.W. Asmar, R. Woo, S.R. Habbal, H. Morgan, Space-time localization of inner heliospheric plasma turbulence using multiple spacecraft radio links. Space Weather 7(12), S12003 (2009)

C.D. Rodgers, Inverse Methods for Atmospheric Sounding: Theory and Practice, vol. 2 (World Scientific, Singapore, 2000)

Z.J. Rong, S. Barabash, G. Stenberg, Y. Futaana, T.L. Zhang, W.X. Wan, Y. Wei, X.D. Wang, L.H. Chai, J. Zhong, The flapping motion of the Venusian magnetotail: Venus Express observations. J. Geophys. Res. Space Phys. 120(7), 5593-5602 (2015). https://doi.org/10.1002/2015JA021317

Z.J. Rong, Y. Ding, J.A. Slavin, J. Zhong, G. Poh, W.J. Sun, Y. Wei, L.H. Chai, W.X. Wan, C. Shen, The magnetic field structure of Mercury's magnetotail. J. Geophys. Res. Space Phys. 123(1), 548-566 (2018). https://doi.org/10.1002/2017JA024923

A. Ruffenach, B. Lavraud, C.J. Farrugia, P. Démoulin, S. Dasso, M.J. Owens, J.A. Sauvaud, A.P. Rouillard, A. Lynnyk, C. Foullon, N.P. Savani, J.G. Luhmann, A.B. Galvin, Statistical study of magnetic cloud erosion by magnetic reconnection. J. Geophys. Res. Space Phys. 120(1), 43-60 (2015). https://doi.org/10.1002/2014JA020628

S. Ruhunusiri, J.S. Halekas, J.R. Espley, C. Mazelle, D. Brain, Y. Harada, G.A. DiBraccio, R. Livi, D.E. Larson, D.L. Mitchell, B.M. Jakosky, G.G. Howes, Characterization of turbulence in the Mars plasma environment with MAVEN observations. J. Geophys. Res. Space Phys. 122(1), 656-674 (2017). https://doi.org/10.1002/2016JA023456

C.T. Russell, J.G. Luhmann, T.E. Cravens, A.F. Nagy, R.J. Strangeway, Venus Upper Atmosphere and Plasma Environment: Critical Issues for Future Exploration. Geophysical Monograph Series, vol. 176 (American Geophysical Union, Washington DC, 2007), pp. 139-156. https://doi.org/10.1029/176GM09

F. Sahraoui, G. Belmont, L. Rezeau, Hamiltonian canonical formulation of Hall-magnetohydrodynamics: toward an application to weak turbulence theory. Phys. Plasmas 10(5), 1325-1337 (2003)

F. Sahraoui, M.L. Goldstein, P. Robert, Y.V. Khotyaintsev, Evidence of a cascade and dissipation of solarwind turbulence at the electron gyroscale. Phys. Rev. Lett. 102(23), 231102 (2009). https://doi.org/ 10.1103/PhysRevLett.102.231102

F. Sahraoui, M.L. Goldstein, G. Belmont, P. Canu, L. Rezeau, Three dimensional anisotropic k spectra of turbulence at subproton scales in the solar wind. Phys. Rev. Lett. 105(13), 131101 (2010). https://doi.org/ 10.1103/PhysRevLett.105.131101

Saito et al. (2021). This journal (for MPPE instrument)

F. Sahraoui, S.Y. Huang, G. Belmont, M.L. Goldstein, A. Rétino, P. Robert, J. De Patoul, Scaling of the electron dissipation range of solar wind turbulence. Astrophys. J. 777(1), 15 (2013). https://doi.org/ 10.1088/0004-637X/777/1/15. 1303.7394

A. Sánchez-Lavega, J. Peralta, J.M. Gomez-Forrellad, R. Hueso, S. Pérez-Hoyos, I. Mendikoa, J.F. Rojas, T. Horinouchi, Y.J. Lee, S. Watanabe, Venus cloud morphology and motions from ground-based images at the time of the Akatsuki orbit insertion. Astrophys. J. Lett. 833(1), L7 (2016). https://doi.org/ 10.3847/2041-8205/833/1/L7. 1611.04318

A. Sánchez-Lavega, S. Lebonnois, T. Imamura, P. Read, D. Luz, The atmospheric dynamics of Venus. Space Sci. Rev. 212(3-4), 1541-1616 (2017). https://doi.org/10.1007/s11214-017-0389-x

F. Santoli, E. Fiorenza, C. Lefevre, D.M. Lucchesi, M. Lucente, C. Magnafico, A. Morbidini, R. Peron, V. Iafolla, ISA, a high sensitivity accelerometer in the interplanetary space. Space Sci. Rev. 216(8), 1-30 (2020). https://doi.org/10.1007/s11214-020-00768-6

D. Schmid, M. Volwerk, F. Plaschke, Z. Vörös, T.L. Zhang, W. Baumjohann, Y. Narita, Mirror mode structures near Venus and comet P/Halley. Ann. Geophys. 32(6), 651-657 (2014). https://doi.org/ 10.5194/angeo-32-651-2014

C.A. Schmidt, J.K. Wilson, J. Baumgardner, M. Mendillo, Orbital effects on Mercury's escaping sodium exosphere. Icarus 207(1), 9-16 (2010). https://doi.org/10.1016/j.icarus.2009.10.017

R. Schunk, A. Nagy, Ionospheres: Physics, Plasma Physics, and Chemistry, 2nd edn. (Cambridge University Press, Cambridge, 2009)

R. Schwenn, Large-scale structure of the interplanetary medium, in Physics of the Inner Heliosphere I (Springer, Berlin, 1990), pp. 99-181 
A. Seiff, J. Schofield, A. Kliore, F. Taylor, S. Limaye, H. Revercomb, L. Sromovsky, V. Kerzhanovich, V. Moroz, M.Y. Marov, Models of the structure of the atmosphere of Venus from the surface to 100 kilometers altitude. Adv. Space Res. 5(11), 3-58 (1985)

O.S. Shalygina, E.V. Petrova, W.J. Markiewicz, N.I. Ignatiev, E.V. Shalygin, Optical properties of the Venus upper clouds from the data obtained by Venus monitoring camera on-board the Venus Express. Planet. Space Sci. 113, 135-158 (2015). https://doi.org/10.1016/j.pss.2014.11.012

D. Shiota, R. Kataoka, Y. Miyoshi, T. Hara, C. Tao, K. Masunaga, Y. Futaana, N. Terada, Inner heliosphere MHD modeling system applicable to space weather forecasting for the other planets. Space Weather 12(4), 187-204 (2014). https://doi.org/10.1002/2013SW000989

M. Shoda, T.K. Suzuki, M. Asgari-Targhi, T. Yokoyama, Three-dimensional simulation of the fast solar wind driven by compressible magnetohydrodynamic turbulence. Astrophys. J. Lett. 880(1), L2 (2019). https://doi.org/10.3847/2041-8213/ab2b45. 1905.11685

J.H. Shue, P. Song, C.T. Russell, J.T. Steinberg, J.K. Chao, G. Zastenker, O.L. Vaisberg, S. Kokubun, H.J. Singer, T.R. Detman, H. Kawano, Magnetopause location under extreme solar wind conditions. J. Geophys. Res. 103(A8), 17691-17700 (1998). https://doi.org/10.1029/98JA01103

J.A. Slavin, R.E. Holzer, Solar wind flow about the terrestrial planets, 1. Modeling bow shock position and shape. J. Geophys. Res. 86(A13), 11401-11418 (1981). https://doi.org/10.1029/JA086iA13p11401

J.A. Slavin, M.H. Acuña, B.J. Anderson, D.N. Baker, M. Benna, S.A. Boardsen, G. Gloeckler, R.E. Gold, G.C. Ho, H. Korth, S.M. Krimigis, R.L. McNutt, J.M. Raines, M. Sarantos, D. Schriver, S.C. Solomon, P. Trávníček, T.H. Zurbuchen, MESSENGER observations of magnetic reconnection in Mercury's magnetosphere. Science 324(5927), 606 (2009). https://doi.org/10.1126/science.1172011

J.A. Slavin, B.J. Anderson, D.N. Baker, M. Benna, S.A. Boardsen, G. Gloeckler, R.E. Gold, G.C. Ho, H. Korth, S.M. Krimigis, R.L. McNutt, L.R. Nittler, J.M. Raines, M. Sarantos, D. Schriver, S.C. Solomon, R.D. Starr, P.M. Trávníček, T.H. Zurbuchen, MESSENGER observations of extreme loading and unloading of Mercury's magnetic tail. Science 329(5992), 665 (2010). https://doi.org/ 10.1126/science. 1188067

J.A. Slavin, B.J. Anderson, D.N. Baker, M. Benna, S.A. Boardsen, R.E. Gold, G.C. Ho, S.M. Imber, H. Korth, S.M. Krimigis, R.L. McNutt Jr., J.M. Raines, M. Sarantos, D. Schriver, S.C. Solomon, P. Trávníček, T.H. Zurbuchen, MESSENGER and Mariner 10 flyby observations of magnetotail structure and dynamics at Mercury. J. Geophys. Res. Space Phys. 117(A1), A01215 (2012a). https://doi.org/ 10.1029/2011JA016900

J.A. Slavin, S.M. Imber, S.A. Boardsen, G.A. DiBraccio, T. Sundberg, M. Sarantos, T. Nieves-Chinchilla, A. Szabo, B.J. Anderson, H. Korth, T.H. Zurbuchen, J.M. Raines, C.L. Johnson, R.M. Winslow, R.M. Killen, R.L. McNutt Jr., S.C. Solomon, MESSENGER observations of a flux-transfer-event shower at Mercury. J. Geophys. Res. Space Phys. 117, A00M06 (2012b). https://doi.org/10.1029/2012JA017926

J.A. Slavin, H.R. Middleton, J.M. Raines, X. Jia, J. Zhong, W.J. Sun, S. Livi, S.M. Imber, G.K. Poh, M. Akhavan-Tafti, J.M. Jasinski, G.A. DiBraccio, C. Dong, R.M. Dewey, M.L. Mays, MESSENGER observations of disappearing dayside magnetosphere events at Mercury. J. Geophys. Res. Space Phys. 124(8), 6613-6635 (2019). https://doi.org/10.1029/2019JA026892

A.W. Smith, J.A. Slavin, C.M. Jackman, G.K. Poh, R.C. Fear, Flux ropes in the Hermean magnetotail: distribution, properties, and formation. J. Geophys. Res. Space Phys. 122(8), 8136-8153 (2017). https://doi.org/10.1002/2017JA024295

R. Soja, M. Sommer, J. Herzog, J. Agarwal, J. Rodmann, R. Srama, J. Vaubaillon, P. Strub, A. Hornig, L. Bausch et al., Characteristics of the dust trail of 67P/Churyumov-Gerasimenko: an application of the IMEX model. Astron. Astrophys. 583, A18 (2015a)

R.H. Soja, J.T. Herzog, M. Sommer, J. Rodmann, J. Vaubaillon, P. Strub, T. Albin, V. Sterken, A. Hornig, L. Bausch et al., Meteor storms and showers with the IMEX model, in Proceedings of the International Meteor Conference (2015b), pp. 66-69

M.L. Stevens, J.C. Kasper, A scale-free analysis of magnetic holes at 1 AU. J. Geophys. Res. Space Phys. 112(A5), A05109 (2007). https://doi.org/10.1029/2006JA012116

T. Sundberg, J.A. Slavin, S.A. Boardsen, B.J. Anderson, H. Korth, G.C. Ho, D. Schriver, V.M. Uritsky, T.H. Zurbuchen, J.M. Raines et al., MESSENGER observations of dipolarization events in Mercury's magnetotail. J. Geophys. Res. Space Phys. 117(A12), A00M03 (2012)

J.M. Sunshine, T.L. Farnham, L.M. Feaga, O. Groussin, F. Merlin, R.E. Milliken, M.F. A'Hearn, Temporal and spatial variability of lunar hydration as observed by the Deep Impact spacecraft. Science 326(5952), 565 (2009). https://doi.org/10.1126/science.1179788

M. Taguchi, T. Fukuhara, T. Imamura, M. Nakamura, N. Iwagami, M. Ueno, M. Suzuki, G.L. Hashimoto, K. Mitsuyama, Longwave infrared camera onboard the Venus climate orbiter. Adv. Space Res. 40(6), 861-868 (2007). https://doi.org/10.1016/j.asr.2007.05.085 
C. Tao, F. Sahraoui, D. Fontaine, J. Patoul, T. Chust, S. Kasahara, A. Retinò, Properties of Jupiter's magnetospheric turbulence observed by the Galileo spacecraft. J. Geophys. Res. Space Phys. 120(4), 2477-2493 (2015). https://doi.org/10.1002/2014JA020749

B. Tapley, F. Flechtner, S. Bettadpur, M. Watkins, The status and future prospect for grace after the first decade, in AGUFM 2013:G32A-01 (2013)

G.I. Taylor, The spectrum of turbulence. Proc. R. Soc. Lond. Ser. A, Math. Phys. Sci. 164(919), 476-490 (1938)

S. Tellmann, M. Pätzold, B. Häusler, M.K. Bird, G.L. Tyler, Structure of the Venus neutral atmosphere as observed by the Radio Science experiment VeRa on Venus Express. J. Geophys. Res., Planets 114(9), E00B36 (2009). https://doi.org/10.1029/2008JE003204

E. Thébault, B. Langlais, J.S. Oliveira, H. Amit, L. Leclercq, A time-averaged regional model of the Hermean magnetic field. Phys. Earth Planet. Inter. 276, 93-105 (2018). https://doi.org/10.1016/ j.pepi.2017.07.001

D.V. Titov, N.I. Ignatiev, K. McGouldrick, V. Wilquet, C.F. Wilson, Clouds and hazes of Venus. Space Sci. Rev. 214(8), 126 (2018). https://doi.org/10.1007/s11214-018-0552-Z

S. Tsuneta, K. Ichimoto, Y. Katsukawa, S. Nagata, M. Otsubo, T. Shimizu, Y. Suematsu, M. Nakagiri, M. Noguchi, T. Tarbell, A. Title, R. Shine, W. Rosenberg, C. Hoffmann, B. Jurcevich, G. Kushner, M. Levay, B. Lites, D. Elmore, T. Matsushita, N. Kawaguchi, H. Saito, I. Mikami, L.D. Hill, J.K. Owens, The Solar Optical Telescope for the Hinode mission: an overview. Sol. Phys. 249(2), 167-196 (2008). https://doi.org/10.1007/s11207-008-9174-z. 0711.1715

C.Y. Tu, E. Marsch, MHD structures, waves and turbulence in the solar wind: observations and theories. Space Sci. Rev. 73(1-2), 1-210 (1995)

J.M. Turner, L.F. Burlaga, N.F. Ness, J.F. Lemaire, Magnetic holes in the solar wind. J. Geophys. Res. 82(13), 1921 (1977). https://doi.org/10.1029/JA082i013p01921

S.G. Turyshev, M. Shao, K.L. Nordtvedt, H. Dittus, C. Laemmerzahl, S. Theil, C. Salomon, S. Reynaud, T. Damour, U. Johann, P. Bouyer, P. Touboul, B. Foulon, O. Bertolami, J. Páramos, Advancing fundamental physics with the Laser Astrometric Test of Relativity. The LATOR mission. Exp. Astron. 27(1-2), 27-60 (2009). https://doi.org/10.1007/s10686-009-9170-9

R.J. Vervack, R.M. Killen, W.E. McClintock, A.W. Merkel, M.H. Burger, T.A. Cassidy, M. Sarantos, New discoveries from MESSENGER and insights into Mercury's exosphere. Geophys. Res. Lett. 43(22), 11,545-11,551 (2016). https://doi.org/10.1002/2016GL071284

M. Volwerk, T.L. Zhang, M. Delva, Z. Vörös, W. Baumjohann, K.H. Glassmeier, First identification of mirror mode waves in Venus' magnetosheath? Geophys. Res. Lett. 35(12), L12204 (2008a). https://doi.org/ 10.1029/2008GL033621

M. Volwerk, T.L. Zhang, M. Delva, Z. Vörös, W. Baumjohann, K.H. Glassmeier, Mirror-mode-like structures in Venus' induced magnetosphere. J. Geophys. Res., Planets 113(15), E00B16 (2008b). https://doi.org/10.1029/2008JE003154

M. Volwerk, M. Delva, Y. Futaana, A. Retinò, Z. Vörös, T.L. Zhang, W. Baumjohann, S. Barabash, Substorm activity in Venus's magnetotail. Ann. Geophys. 27(6), 2321-2330 (2009). https://doi.org/ 10.5194/angeo-27-2321-2009

M. Volwerk, M. Delva, Y. Futaana, A. Retinò, Z. Vörös, T.L. Zhang, W. Baumjohann, S. Barabash, Corrigendum to "Substorm activity in Venus's magnetotail". Ann. Geophys. 28(10), 1877-1878 (2010). https://doi.org/10.5194/angeo-28-1877-2010; Ann. Geophys. 27, 2321-2330 (2009). https://doi.org/ 10.5194/angeo-27-2321-2009

M. Volwerk, D. Schmid, B.T. Tsurutani, M. Delva, F. Plaschke, Y. Narita, T. Zhang, K.H. Glassmeier, Mirror mode waves in Venus's magnetosheath: solar minimum vs. solar maximum. Ann. Geophys. 34(11), 1099-1108 (2016). https://doi.org/10.5194/angeo-34-1099-2016

M. Volwerk, C. Goetz, F. Plaschke, T. Karlsson, D. Heyner, B. Anderson, On the magnetic characteristics of magnetic holes in the solar wind between Mercury and Venus. Ann. Geophys. 38(1), 51-60 (2020). https://doi.org/10.5194/angeo-38-51-2020

A. Vourlidas, R.A. Howard, S.P. Plunkett, C.M. Korendyke, A.F.R. Thernisien, D. Wang, N. Rich, M.T. Carter, D.H. Chua, D.G. Socker, M.G. Linton, J.S. Morrill, S. Lynch, A. Thurn, P. Van Duyne, R. Hagood, G. Clifford, P.J. Grey, M. Velli, P.C. Liewer, J.R. Hall, E.M. DeJong, Z. Mikic, P. Rochus, E. Mazy, V. Bothmer, J. Rodmann, The Wide-Field Imager for Solar Probe Plus (WISPR). Space Sci. Rev. 204(1-4), 83-130 (2016). https://doi.org/10.1007/s11214-014-0114-y

R.C. Whitten, B. Baldwin, W.C. Knudsen, K.L. Miller, K. Spenner, The Venus ionosphere at grazing incidence of solar radiation: transport of plasma to the night ionosphere. Icarus 51(2), 261-270 (1982). https://doi.org/10.1016/0019-1035(82)90082-3 
J. Wicht, D. Heyner, Mercury's magnetic field in the MESSENGER era, in Planetary Geodesy and Remote Sensing (2014), pp. 223-262

T.W. Williams, N. Ottenstein, M. Farahmand, E. Palmer, Initial satellite formation flight results from the magnetospheric multiscale mission, in AIAA/AAS Astrodynamics Specialist Conference (2016), p. 5505

V. Wilquet, R. Drummond, A. Mahieux, S. Robert, A.C. Vandaele, J.L. Bertaux, Optical extinction due to aerosols in the upper haze of Venus: four years of SOIR/VEX observations from 2006 to 2010. Icarus 217(2), 875-881 (2012). https://doi.org/10.1016/j.icarus.2011.11.002

C.F. Wilson, S. Guerlet, P.G.J. Irwin, C.C.C. Tsang, F.W. Taylor, R.W. Carlson, P. Drossart, G. Piccioni, Evidence for anomalous cloud particles at the poles of Venus. J. Geophys. Res., Planets 113(12), E00B13 (2008). https://doi.org/10.1029/2008JE003108

R.M. Winslow, C.L. Johnson, B.J. Anderson, D.J. Gershman, J.M. Raines, R.J. Lillis, H. Korth, J.A. Slavin, S.C. Solomon, T.H. Zurbuchen, M.T. Zuber, Mercury's surface magnetic field determined from proton-reflection magnetometry. Geophys. Res. Lett. 41(13), 4463-4470 (2014). https://doi.org/ 10.1002/2014GL060258

R.M. Winslow, N. Lugaz, L.C. Philpott, N.A. Schwadron, C.J. Farrugia, B.J. Anderson, C.W. Smith, Interplanetary coronal mass ejections from MESSENGER orbital observations at Mercury. J. Geophys. Res. Space Phys. 120(8), 6101-6118 (2015). https://doi.org/10.1002/2015JA021200

R.M. Winslow, N. Lugaz, N.A. Schwadron, C.J. Farrugia, W. Yu, J.M. Raines, M.L. Mays, A.B. Galvin, T.H. Zurbuchen, Longitudinal conjunction between MESSENGER and STEREO A: development of ICME complexity through stream interactions. J. Geophys. Res. Space Phys. 121(7), 6092-6106 (2016). https://doi.org/10.1002/2015JA022307

D. Winterhalter, M. Neugebauer, B.E. Goldstein, E.J. Smith, B.T. Tsurutani, S.J. Bame, A. Balogh, Magnetic holes in the solar wind and their relation to mirror mode structures. Space Sci. Rev. 72(1-2), 201-204 (1995). https://doi.org/10.1007/BF00768780

S.D. Xiao, T.L. Zhang, Z. Vörös, Magnetic fluctuations and turbulence in the Venusian magnetosheath downstream of different types of bow shock. J. Geophys. Res. Space Phys. 123(10), 8219-8226 (2018). https://doi.org/10.1029/2018JA025250

S. Yagitani, M. Ozaki, F. Sahraoui, L. Mirioni, M. Mansour, G. Chanteur, C. Coillot, S. Ruocco, V. Leray, M. Hikishima et al., Measurements of magnetic field fluctuations for plasma wave investigation by the search coil magnetometers (SCM) onboard BepiColombo Mio (Mercury magnetospheric orbiter). Space Sci. Rev. 216(7), 1-26 (2020). https://doi.org/10.1007/s11214-020-00734-2

A. Yamazaki, M. Yamada, Y.J. Lee, S. Watanabe, T. Horinouchi, S.y. Murakami, T. Kouyama, K. Ogohara, T. Imamura, T.M. Sato, Y. Yamamoto, T. Fukuhara, H. Ando, K.i. Sugiyama, S. Takagi, H. Kashimura, S. Ohtsuki, N. Hirata, G.L. Hashimoto, M. Suzuki, C. Hirose, M. Ueno, T. Satoh, T. Abe, N. Ishii, M. Nakamura, Ultraviolet imager on Venus orbiter Akatsuki and its initial results. Earth Planets Space 70(1), 23 (2018). https://doi.org/10.1186/s40623-017-0772-6

H. Yano, T. Hirai, Revealing exo-zody and exo-planets from solar system dust measurements: ALADDIN-2 for the Solar Power Sail mission, in 41st COSPAR Scientific Assembly, vol. 41 (2016)

L.V. Zasova, I.A. Khatountsev, V.I. Moroz, N.I. Ignatiev, Structure of the Venus middle atmosphere: Venera 15 Fourier spectrometry data revisited. Adv. Space Res. 23(9), 1559-1568 (1999). https://doi.org/ 10.1016/S0273-1177(99)00169-6

L.V. Zasova, N. Ignatiev, I. Khatuntsev, V. Linkin, Structure of the Venus atmosphere. Planet. Space Sci. 55(12), 1712-1728 (2007). https://doi.org/10.1016/j.pss.2007.01.011

T.L. Zhang, M. Delva, W. Baumjohann, M. Volwerk, C.T. Russell, S. Barabash, M. Balikhin, S. Pope, K.H. Glassmeier, C. Wang, K. Kudela, Initial Venus Express magnetic field observations of the magnetic barrier at solar minimum. Planet. Space Sci. 56(6), 790-795 (2008). https://doi.org/10.1016/ j.pss.2007.10.013

T.L. Zhang, Q.M. Lu, W. Baumjohann, C.T. Russell, A. Fedorov, S. Barabash, A.J. Coates, A.M. Du, J.B. Cao, R. Nakamura, W.L. Teh, R.S. Wang, X.K. Dou, S. Wang, K.H. Glassmeier, H.U. Auster, M. Balikhin, Magnetic reconnection in the near Venusian magnetotail. Science 336(6081), 567 (2012). https://doi.org/10.1126/science.1217013

J. Zhong, W.X. Wan, J.A. Slavin, Y. Wei, R.L. Lin, L.H. Chai, J.M. Raines, Z.J. Rong, X.H. Han, Mercury's three-dimensional asymmetric magnetopause. J. Geophys. Res. Space Phys. 120(9), 7658-7671 (2015). https://doi.org/10.1002/2015JA021425

J. Zhong, Y. Wei, Z. Pu, X. Wang, W. Wan, J. Slavin, X. Cao, J. Raines, H. Zhang, C. Xiao et al., MESSENGER observations of rapid and impulsive magnetic reconnection in Mercury's magnetotail. Astrophys. J. Lett. 860(2), L20 (2018)

J. Zhong, Q. Zong, Y. Wei, J. Slavin, X. Cao, Z. Pu, X. Wang, S. Fu, J. Raines, W. Wan, MESSENGER observations of giant plasmoids in Mercury's magnetotail. Astrophys. J. Lett. 886(2), L32 (2019) 
J. Zhong, L.C. Lee, X.G. Wang, Z.Y. Pu, J.S. He, Y. Wei, W.X. Wan, Multiple X-line reconnection observed in Mercury's magnetotail driven by an interplanetary coronal mass ejection. Astrophys. J. Lett. 893(1), L11 (2020a). https://doi.org/10.3847/2041-8213/ab8380

J. Zhong, Y. Wei, L.C. Lee, J.S. He, J.A. Slavin, Z.Y. Pu, H. Zhang, X.G. Wang, W.X. Wan, Formation of macroscale flux transfer events at Mercury. Astrophys. J. Lett. 893(1), L18 (2020b). https://doi.org/ $10.3847 / 2041-8213 / \mathrm{ab} 8566$ 